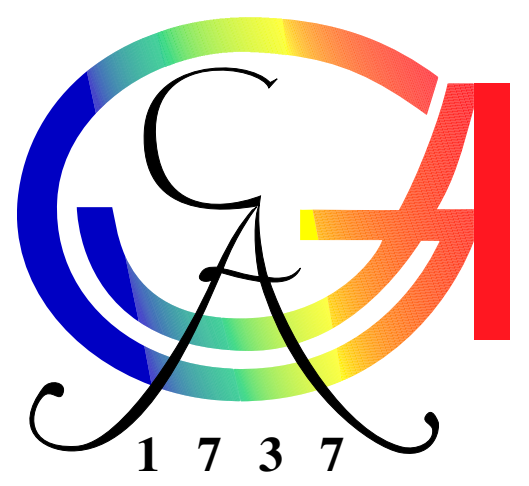

\title{
Untersuchungen zur IR-Laser-Ablation in Wasser
}

\author{
Dissertation \\ zur Erlangung des Doktorgrades der \\ Mathematisch-Naturwissenschaftlichen Fakultäten der \\ Georg-August-Universität zu Göttingen \\ vorgelegt von \\ Tobias Brendel \\ aus \\ Verden
}

Göttingen 2004 
D7

Referent: Prof. Dr. Werner Lauterborn

Korreferent: Prof. Dr. Tim Salditt

Tag der mündlichen Prüfung: 10. Juni 2004 


\section{Inhaltsverzeichnis}

1. Einleitung 5

2. Grundlagen 9

2.1. Anwendungen und Eigenschaften von Infrarot-Lasern . . . . . . . . . . . 9

2.2. Photothermische und photoakustische Energiewandlung . . . . . . . . . . 14

2.2.1. Photothermische Energiewandlung . . . . . . . . . . . . . . 14

2.2.2. Photoakustische Energiewandlung . . . . . . . . . . . 20

2.3. Blasenbildung durch Kavitation und Verdampfung . . . . . . . . . . . 22

2.3.1. Blasenentstehung aus einem Nukleationskeim . . . . . . . . 23

2.3.2. Heterogene Nukleation an einem Oberflächendefekt . . . . . . 28

3. Darstellung der thermoelastischen Schallentwicklung 31

3.1. Übersicht . . . . . . . . . . . . . . . . . . 31

3.2. Lösung der photoakustischen Wellengleichung . . . . . . . . . . 35

3.2.1. Thermische Nichtlinearitäten bei der Tm- und Ho-Laseranregung 44

3.3. Berechnung zum Gaußschen Laserstrahl . . . . . . . . . . . . . . . 46

3.3.1. Schallentwicklung auf der Strahlachse . . . . . . . . . . . . . 46

3.3.2. Schallentwicklung in der Eintrittsebene . . . . . . . . . . . 50

3.4. Berechnung zum fasergeführten Laserstrahl . . . . . . . . . . . . . 51

3.4.1. Schallentwicklung in der Eintrittsebene . . . . . . . . . . 52 
4. Implikationen für den thermodynamischen Zustandsverlauf 55

4.1. Schallentwicklung zum Gaußschen Laserstrahl _ . . . . . . . . . . . . . 56

4.1.1. Phasendiagramm zur Anregung . . . . . . . . . . . . . . 60

4.1.2. Charakteristische Schallfeldgrößen . . . . . . . . . . . 65

4.2. Abhängigkeit von den Anregungsparametern . . . . . . . . . . . . . 66

4.2.1. Betrachtung bei konstanter Erhitzung . . . . . . . . . . . . 67

4.2.2. Betrachtung bei konstanter Pulsenergie . . . . . . . . . . . 76

4.3. Fasergeführte Laseranregung . . . . . . . . . . . . . . . . . . . 80

5. Modell und Experiment im Vergleich 85

5.1. Startzeitpunkte der Ablation _. . . . . . . . . . . . . . . . . 86

5.1.1. Ablationsbeginn und Schallentwicklung . . . . . . . . . . . . . 90

5.1.2. Schallmessungen . . . . . . . . . . . . . . 95

6. Zusammenfassung 99

A. Anhang 102

A.1. Lösung der Poisson'schen Formel-Integrale . . . . . . . . . . . . . . . . 102

A.2. Geometrische Betrachtungen zur Schalldetektion . . . . . . . . . . 104

A.3. Wärmeleitung an der Grenzfläche Quarz-Wasser . . . . . . . . . . . . 106

Literaturverzeichnis

110 


\section{Einleitung}

Der Wasseranteil von biologischem Weichgewebe liegt bei durchschnittlich 70 Prozent [19]. Für die chirurgische Gewebeentfernung (lat. „Ablatio“) sind Infrarot-Laserquellen besonders geeignet, weil Wasser im infraroten Spektralbereich eine hohe Absorption aufweist. Die Energiedeponierung bleibt hierdurch auf eine kleinvolumige optische Zone beschränkt, so daß mit moderaten Energien eine effiziente Ablation erreicht werden kann. Der direkte Weg zum Gewebeabtrag verläuft dabei über die Verdampfung der Gewebebestandteile. Für die Anregung mit freilaufenden oder kontinuierlich betriebenen IR-Lasern ist dies der dominierende Ablationsmechanismus, dessen Thermodynamik weitgehend verstanden ist $[10,24,81]$.

Neben der Verdampfung gibt es eine Reihe zusätzlicher Effekte, die durch die laserinduzierte Erhitzung des bestrahlten Gewebevolumens ausgelöst werden (Abbildung 1.1). Sie können den Gewebeabtrag unterstützen oder zu einer Schädigung am umliegenden Gewebe führen. So hat z.B. die Dynamik einer sich bildenden Dampfblase das Potential zu mechanischen Gewebezerreißungen während der Blasenexpansion (Abbildung 1.2) und des Blasenkollapses (Abbildung 1.4).

Erfolgt die Laseranregung auf sehr kurzen Zeitskalen, so dehnt sich das angeregte Gewebevolumen nicht so schnell aus wie es erhitzt wird. Aus dieser thermischen Kompression entsteht eine sog. thermoelastische Druckwelle, die sich beugungsbedingt in eine bipolare Schallwelle mit Druck- und Zuganteilen umwandelt .

Die thermoelastische Schallentwicklung kann Amplituden von mehreren 100 bar erreichen, wenn die Anregung nicht länger als einige 10 bis $100 \mathrm{~ns}$ dauert. Dies entspricht den typischen Pulsdauern gütegeschalteter IR-Lasersysteme. Eine Übersicht über die IRLaserquellen, die in der experimentellen Praxis zur Verfügung stehen, gibt Kapitel 2. Im Vordergrund stehen dabei solche Laser, die für einen Einsastz in der minimal-invasiven Chirurgie geeignet sind. Weiter werden in Kapitel 2 die Grundlagen der photothermischen 


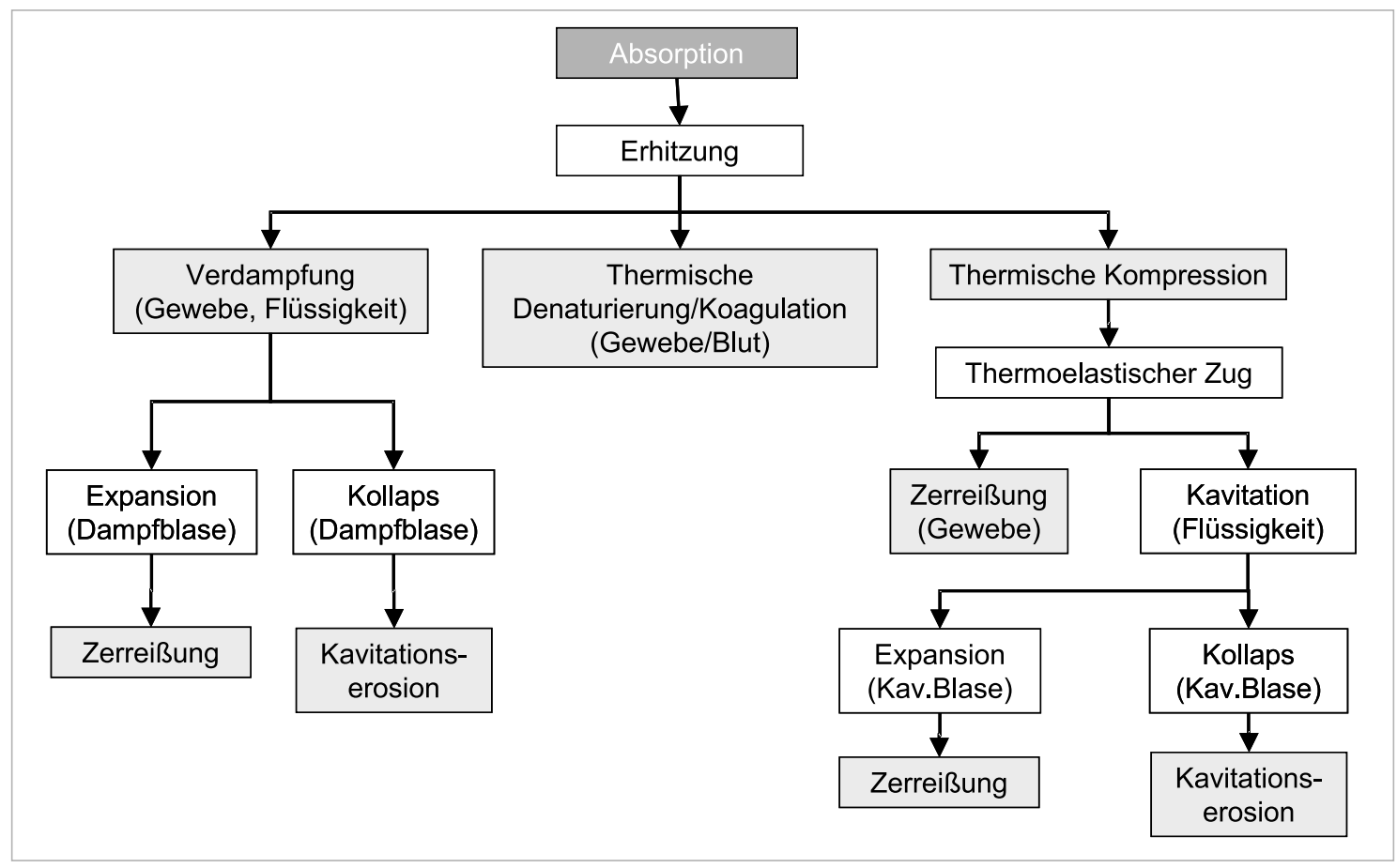

Abb. 1.1: Wirkungsbaum der IR-Laser-Ablation von Gewebe. Die schädigungswirksamen Effekte sind hellgrau unterlegt, photochemische Effekte [36] sind nicht berücksichtigt.

und photoakustischen Energiewandlung erörtert sowie die Mechanismen der verdampfungs- oder kavitationsbedingten Blasenbildung dargestellt.

In der Literatur findet sich eine größere Zahl von Arbeiten, die sich experimentell und theoretisch mit der gepulsten Laserablation in Gewebe, Wasser oder wässrigen Lösungen beschäftigen. Wurde auch die thermoelastische Schallentwicklung untersucht, so stand zumeist die direkte Wechselwirkung der Schallwelle mit dem Gewebe im Vordergrund $[17,24,25,62,65,67]$. Weniger Beachtung findet bisher die Frage, welche Auswirkungen die Änderungen in der Zustandsgröße Druck auf den simultan ablaufenden Verdampfungsprozeß haben, etwa im Hinblick auf eine transiente Siedepunktsverschiebung $[1,10]$. Es ist das Ziel der vorliegenden Arbeit, dieser Frage näher nachzugehen. Dazu wird in Kapitel 3 ein Druckmodell entwickelt, an dem sich das Verhalten des thermoelastischen Schallfeldes als Funktion der wichtigsten Anregungsparameter wie Pulsenergie, Pulsdauer, Strahldurchmesser und Absorption studieren läßt. Das Modell ist gültig für eine lineare Schallausbreitung in Wasser und wird für zwei Strahlprofile gerechnet: für das Top-hat-Profil eines fasergeführten Lasersstrahls und für ein Gaußsches Strahlprofil entsprechend der transversalen Laser-Grundmode TEM $\mathrm{T}_{00}$. 


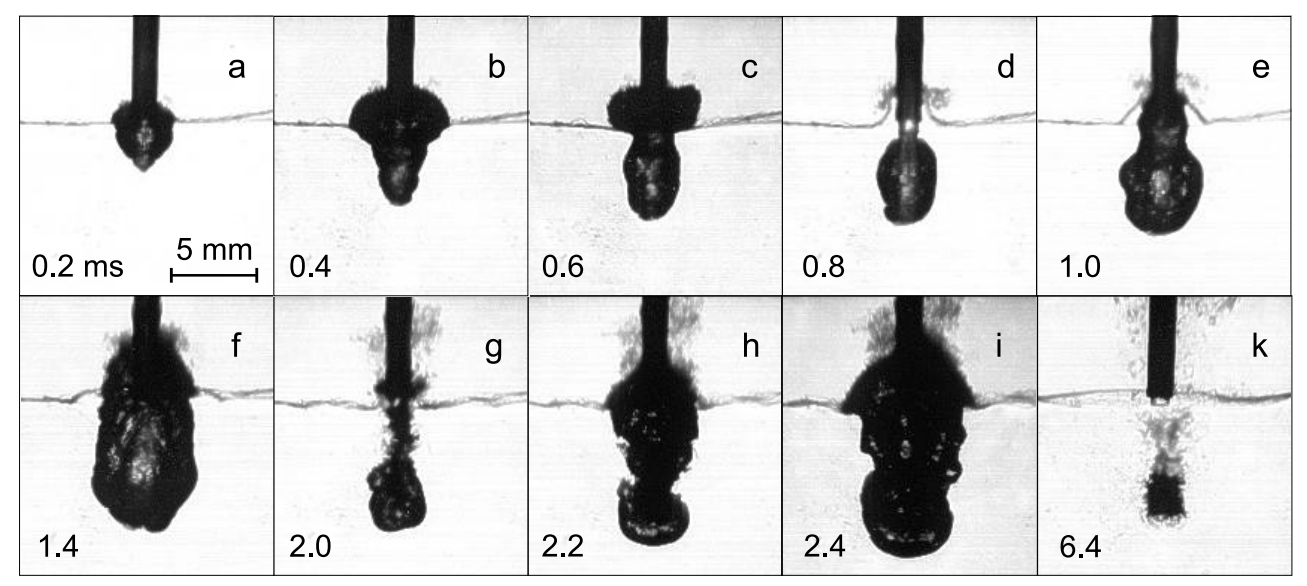

Abb. 1.2: Ablation eines transparenten Kunststoff-Gewebephantoms in wässriger Umgebung durch einen fasergeführten, freilaufenden Holmium-Laserpuls (Cr:Tm:Ho:YAG, $\lambda=2.12 \mu \mathrm{m}$, Pulsdauer: $2.2 \mathrm{~ms}$ ) [12]. Die strahlungsführende Quarzfaser ist lose auf die Oberfläche des Gewebephantoms aufgesetzt. In den Abbildungen d und e ist deutlich das Aufreißen der Materialoberfläche durch die Dampfblasen zu sehen. Weitere nachgewiesene Risse im Inneren der Probe sind auf den Abbildungen nicht zu erkennen.

In Kapitel 4 erfolgt die Auswertung des Modells nach den Abhängigkeiten der genannten Anregungsparameter. Die Ergebnisse werden mit dem zeitlichen Verlauf der Laseranregung und der Temperaturentwicklung korreliert, um so den thermodynamischen Zustandsverlauf der angeregten Probe nachzeichnen oder auch vorhersagen zu können. Die Güte der Modellvorhersagen wird in Kapitel 5 durch Vergleich mit experimentellen Ergebnissen aus der Literatur geprüft. Die Arbeit schließt mit einer Zusammenfassung der Resultate in Kapitel 6. 

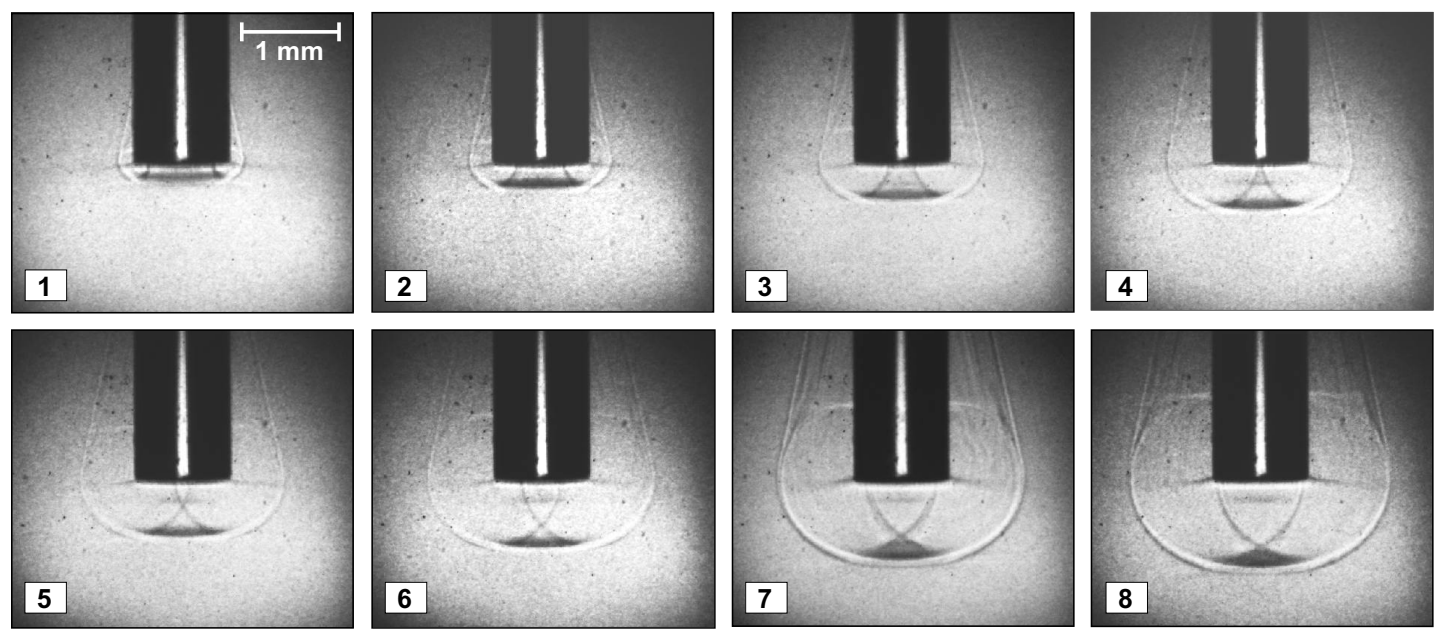

Abb. 1.3: Schlierenoptische Aufnahmen eines thermoelastischen Schallfeldes, das in einer Farbstofflösung (Ethanol + Rhodamin $6 \mathrm{G}$ ) durch einen gütegeschalteten, fasergeführten Nd:YAG-Laserpuls erzeugt wurde $(\lambda=532 \mathrm{~nm}$, Pulsdauer: $14 \mathrm{~ns})$. Das erste Bild entstand $100 \mathrm{~ns}$ nach Pulsapplikation, die weiteren Bilder im Abstand von $100 \mathrm{~ns}$ (Belichtungszeit: 5ns). Alle Aufnahmen aus [6].

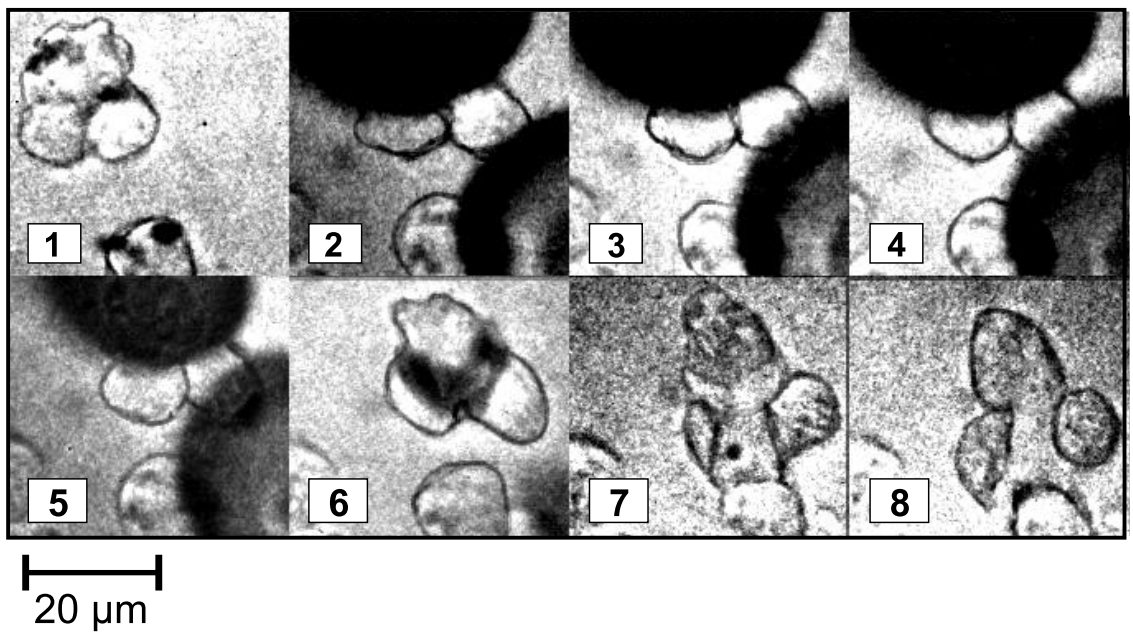

Abb. 1.4: Beispiel einer Zellschädigung durch kollabierende Blasen: Das erste Bild zeigt einen ungestörten Cluster aus drei Fibroblastzellen. In Bild 2 sind als dunkle Schatten zwei Kontrastmittelblasen zu erkennen, die ca. $10 \mu$ s zuvor durch einen funkeninduzierten Schallimpuls zur Expansion angeregt wurden. Die folgenden Bilder (Zeitabstand: $1 \mu \mathrm{s}$, Belichtungszeit: $200 \mathrm{~ns}$ ) zeigen die Kollapsphase der Blasen, in der es zu einer starken starken Deformierung der Zellen kommt (Bild 6). Im letzten Bild weist die untere linke Zelle eine deutlich Ruptur auf. Die Aufnahmen sind einer Arbeit von B. Wolfrum, T. Kurz, R. Mettin und W. Lauterborn [82] entnommen. 


\section{Grundlagen}

\subsection{Anwendungen und Eigenschaften von Infrarot- Lasern}

Für die IR-Laser-Ablation stehen verschiedene Laserquellen zur Verfügung, die in Abbildung 2.1 nach ihrer Emissionswellenlänge in das Absorptionsspektrum von Wasser eingeordnet sind. Im einzelnen handelt es sich um den Erbium- (Er:YAG, $\lambda=2.94 \mu \mathrm{m})$, den Kohlenstoffdioxid- $\left(\mathrm{CO}_{2}, \lambda=10.6 \mu \mathrm{m}\right)$, sowie den Thulium- $(\mathrm{Cr}: \mathrm{Tm}: Y A G, \lambda=2.01 \mu \mathrm{m})$ und den Holmium-Laser (Cr:Tm:Ho:YAG, $\lambda=2.1 \mu \mathrm{m}$ ), im folgenden kurz Er-, $\mathrm{CO}_{2-}$, Tm- und Ho-Laser genannt. Die Emissionswellenlängen des Tm- und des Ho-Lasers unterscheiden sich hierbei nur wenig. Da sie jedoch im Flankenbereich des lokalen Absorptionsmaximums bei $1.94 \mu \mathrm{m}$ liegen, differiert die Absorption zwischen beiden um mehr als einen Faktor zwei (Unterabschnitt 2.2.1).

Die höchste Absorption erzielt die Strahlung des Er-Lasers, dessen Emissionswellenlänge exakt im Absorptionsmaximum von Wasser bei $2.94 \mu \mathrm{m}$ liegt. Bei einer optischen Eindringtiefe von nur etwa einem Mikrometer wird der Er-Laser z.B. in der Dermatologie zur gezielten Abtragung einzelner Hautschichten eingesetzt [58]. Für die Abtragung größerer Gewebemengen eignet sich der $\mathrm{CO}_{2}$-Laser, der einen gepulsten oder kontinuierlichen Betrieb bei hohen Laserleistungen erlaubt. Das Einsatzgebiet von Er- und $\mathrm{CO}_{2}$-Lasern ist jedoch dadurch eingeschränkt, daß ihre Strahlung nicht mittels Glasfasern geführt werden kann: Die hohe Absorption beider Laserwellenlängen in Wasser setzt sich an den $\mathrm{OH}^{-}$-Ionen fort, die in Spuren in jedem optischem Glas oder Quarz vorhanden sind. Zur Führung der Laserstrahlung im Raum werden daher technisch aufwendige Spiegelgelenkarme benötigt. 


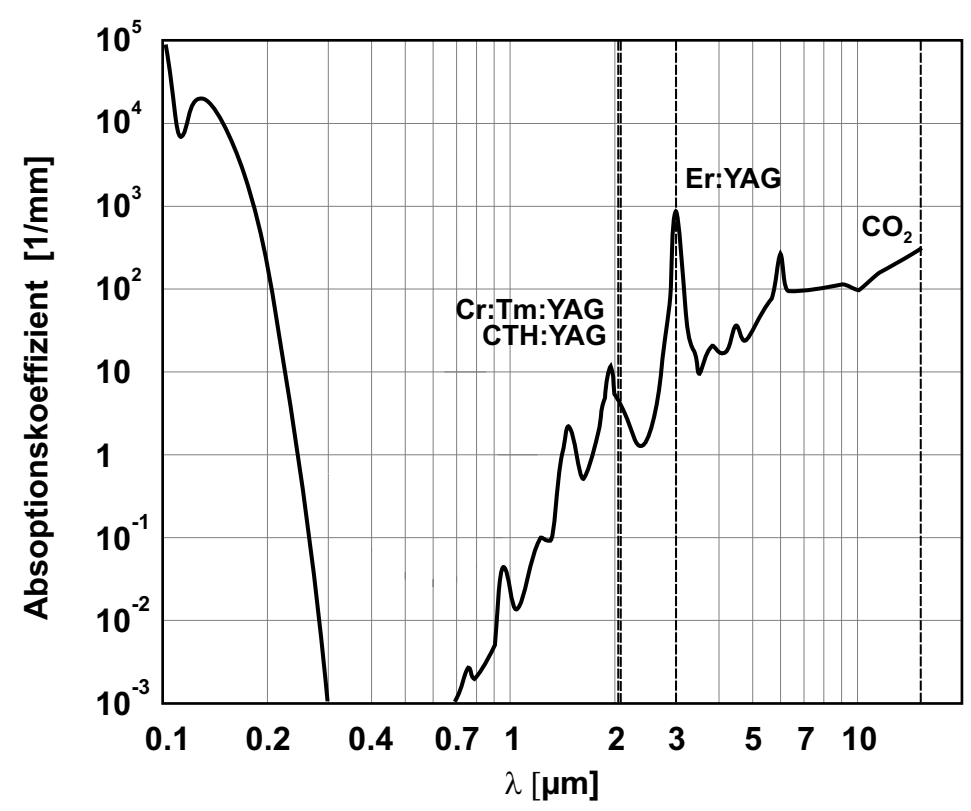

Abb. 2.1: Absorptionsspektrum von Wasser in doppelt-logarithmischer Auftragung (nach J.-L. Boulnois [5]).

Einen guten Kompromiß zwischen Fasergängigkeit und Ablationseffizienz bieten die Absorptionen bei den Wellenlängen des Tm- und des Ho-Lasers. Für diese Laser eröffnet sich der Einsatz in der minimal-invasiven Chirurgie, indem ein flexibler Katheter oder ein Endoskop mit einer (Multimode-)Quarzglasfaser ausgerüstet und die Laserstrahlung so zum Zielgewebe im Körperinneren geführt wird. Das hohe Anwendungspotential dieses Verfahrens belegen Beispiele aus der Ureterotomie (Entfernung von Harnleiterverschlüssen) [11], der Myokard-Revaskularisation (durch Wundreiz stimulierte Gefäßneubildungen im Herzmuskelgewebe) [12] oder der arthroskopischen Chirurgie an Gelenkknorpeln [74]. In diesen Anwendungen findet der Ablationsvorgang stets in flüssiger Umgebung statt, etwa in Blut, Gewebs- oder Gelenkflüssigkeit.

\section{Betriebsarten}

Die gängigen Tm- und Ho-Lasersysteme sind blitzlampengepumpt und werden freilaufend oder gütegeschaltet betrieben [12, 18, 33]. Für einen kontinuierlichen Betrieb stehen diodengepumpte Lasersysteme zur Verfügung [22]. Im freilaufenden und kontinuierlichen Betrieb wird eine stabile Resonatorkonfiguration aufgebaut und die Resonatorgüte konstant gehalten. 
Charakteristisch für den freilaufenden Betrieb der Tm- und Ho-Lasersysteme ist ein ausgeprägtes Spiking während der Emission (Abbildung 2.2) [8, 24].

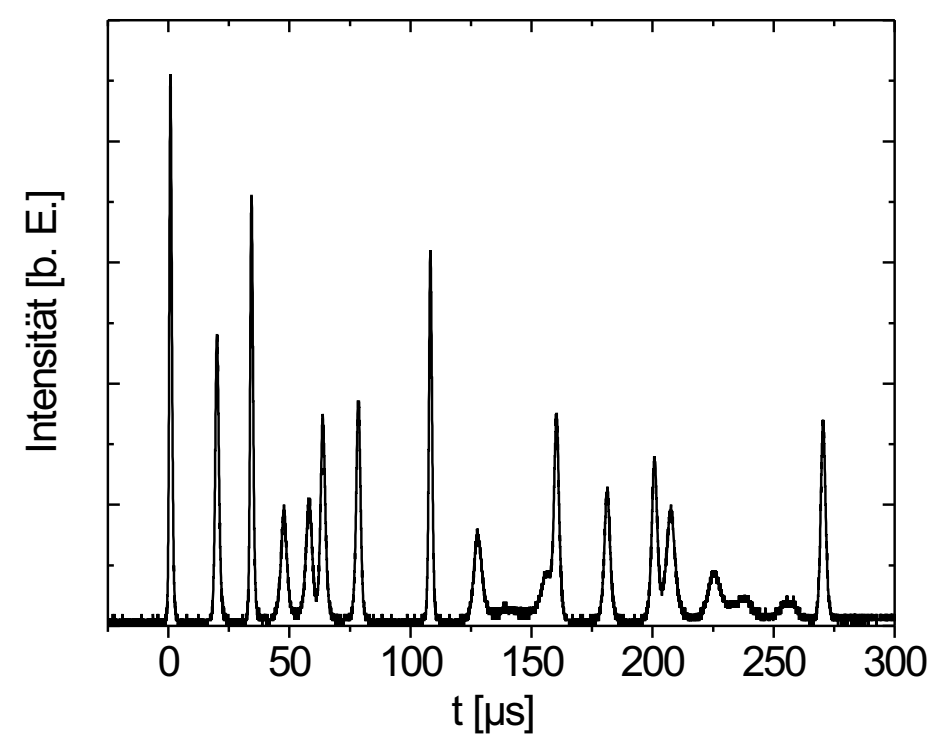

Abb. 2.2: Pulsform eines Tm-Lasers im freilaufenden Betrieb [8]. Die Breite der Einzelspikes beträgt $\geq 1.5 \mu$ s (FWHM).

Bei der Güteschaltung wird der Resonator um ein Element (z.B. Pockelszelle, Drehspiegel) ergänzt, mit dem die Resonatorgüte zu Beginn des Pumpvorganges niedrig gehalten wird, um die kohärente Emission zu verhindern und statt dessen die Pumpenergie im oberen Laserniveau zu speichern. Ist eine ausreichend hohe Inversion erreicht, so wird die Resonatorgüte heraufgesetzt, worauf es zur Emission eines kurzen, energiereichen Laserpulses kommt. Die zeitlichen Pulsformen $g(t)$ des gütegeschalteten Tm- und Ho-Lasers lassen sich in guter Näherung durch eine Gaußkurve der Form

$$
g(t) \propto \exp \left[-\ln 2\left(\frac{2 t}{t_{\mathrm{p}}}\right)^{2}\right]
$$

beschreiben, mit $t_{\mathrm{p}}$ als Pulsdauer entsprechend der Halbwertsbreite (FWHM - full width at half maximum). Die Übereinstimmung mit den Laserpulsformen ist in Abbildung 2.3 zu sehen: Das Profil des Tm-Laserpulses wird durch die Gaußkurve fast identisch wiedergegeben, im Fall des Ho-Laserpulses weicht die Kurve zum Pulsende leicht ab. Die Abweichung macht in der Pulsenergie $\int g(t) \mathrm{d} t$ einen Unterschied von weniger als $5 \%$ gegenüber dem realen Laserpuls aus. Abhängig von der jeweiligen Resonatorkonfiguration und Art der Güteschaltung, der Pumpenergie, Kristalldotierung und zahlreichen anderen Faktoren liegen die Pulsdauern des gütegeschalteten Ho-Lasers zwischen 30 ns und 

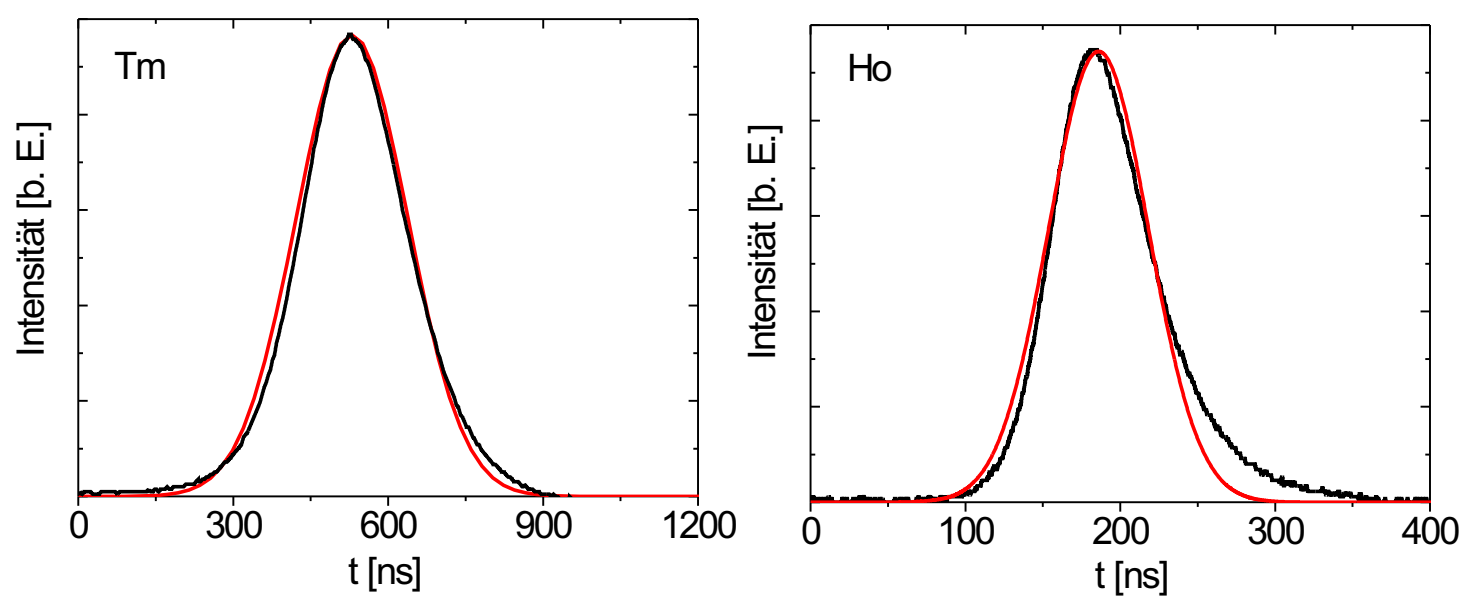

Abb. 2.3: Zeitliche Pulsprofile eines blitzlampengepumpten Tm- und Ho-Lasersystems im gütegeschalteten Betrieb [9]. Dazu angepaßte Gaußkurven $g(t)$ mit gleicher Halbwertsbreite (rot).

$200 \mathrm{~ns}$ [9, 24, 33, 57], die des gütegeschalteten Tm-Lasers zwischen $200 \mathrm{~ns}$ und $500 \mathrm{~ns}$ [9, 10, 33, 57]. Die Pulsenergien betragen typischerweise 30-100 mJ [10, 24, 57].

Mit der Faserführung der IR-Laserstrahlung für minimal-invasive Anwendungen ist die Bestrahlungsgeometrie auf ein Top-hat-Profil festgelegt. Aufgrund der Kohärenz des Laserlichtes ist die Bestrahlungsstärke am Faseraustritt nicht homogen, sondern statistisch moduliert ("verspeckelt") [48]. Für gütegeschaltete Tm- und Ho-Laserpulse wurden an der Austrittsfläche einer Standard-Multimodefaser (Länge: 2 - 4m, Kern- $\oslash$ : 300-400 $\mu \mathrm{m}$ ) lokale Speckelintensitäten vom zwei- bis vierfachen der mittleren Intensität beobachtet $[10,24]$. Die Speckelbildung resultiert aus der Interferenz der transversalen Fasermoden, die ihrerseits aus den eingekoppelten transversalen Lasermoden $\mathrm{TEM}_{\mathrm{nm}}$ hervorgehen. Da die $\mathrm{TEM}_{\mathrm{nm}}$-Moden sukzessive in einem Laserresonator anlaufen, können die Speckel zeitlich und räumlich modulieren [10]. Im bestrahlten Probevolumen entsteht hierdurch eine irreguläre Temperaturverteilung (Abbildung 2.4).

In vorangegangenen experimentellen Untersuchungen hat der Verfasser dieser Arbeit gemeinsam mit R. Brinkmann vorgeschlagen, im Laborversuch auf eine Faserführung der IR-Laserstrahlung zu verzichten, um die Gewebe- bzw. Wasserablation unter wohldefinierten Bestrahlungsbedingungen untersuchen zu können [7]. Als Strahlungsquelle wurde ein gütegeschaltetes Lasersystem verwendet, dessen Resonator auf einen Betrieb in der Gaußschen Grundmode TEM $_{00}$ optimiert war. In den Resonator konnten wahlweise Tm- oder Ho-Lasermedien eingesetzt werden. Der Gaußsche Laserstrahl wurde 


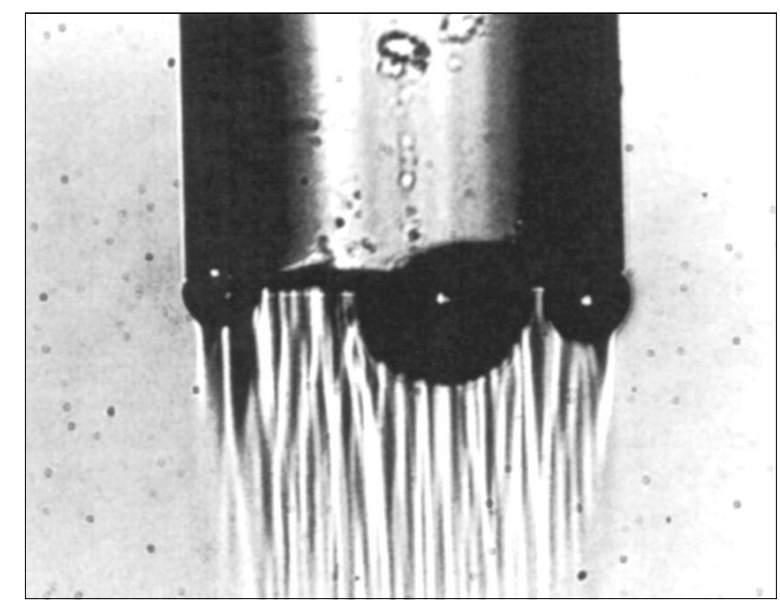

Abb. 2.4: Seitliche Aufnahme (Belichtungszeit: 15ns) eines Quarzfaserendes in Wasser [10], ca. $20 \mu$ s nach Bestrahlungsbeginn durch einen freilaufenden Thuliumlaser (Pulsdauer: $300 \mu \mathrm{s})$. Neben den ersten Verdampfungsblasen sind deutlich thermische Schlieren als Folge der Speckelmodulationen im Laserstrahlprofil zu erkennen.

über Spiegel zu einer Küvette mit vorgesetztem Quarzprisma geführt. In der Küvette befand sich die untersuchte Wasserprobe, die durch das Prisma hindurch bestrahlt wurde. Die eingetauchte Prismenoberfläche modellierte so ein eingetauchtes Quarzfaserende ana$\log$ zur Bestrahlungssituation in der minimal-invasiven Anwendung. Aufgrund der wohldefinierten Bestrahlungsbedingungen, die mit dem Gaußschen Laserstrahl erzeugt werden können, eignet sich die Gaußsche Bestrahlungsgeometrie besonders für vergleichende Modellbetrachtungen zur laserinduzierten Temperatur- und Schallentwicklung (Kapitel 3, 4). Die Bestrahlung $H(x, y)\left[\mathrm{mJ} / \mathrm{mm}^{2}\right]$ eines radialsymmetrischen Gaußstrahls ist durch die Pulsenergie $E$ und den Strahlradius $w$ gemäß

$$
H(x, y)=H_{0} \exp \left(-2 \frac{x^{2}+y^{2}}{w^{2}}\right), \quad \text { mit } \quad H_{0}=\frac{2 E}{\pi w^{2}}
$$

eindeutig bestimmt. Der Strahlradius ist Messungen leicht zugänglich, etwa durch Anwendung des „Messerschneiden“-Verfahrens [7]. 


\subsection{Photothermische und photoakustische Ener- giewandlung}

In diesem Abschnitt werden die theoretischen Zusammenhänge zwischen der applizierten Bestrahlung und der zugehörigen Änderung von Temperatur und Druck hergestellt. Bei der photothermischen Energiewandlung ist dabei die Temperaturabhängigkeit der Absorption zu berücksichtigen (Unterabschnitt 2.2.1), bei der photoakustischen Energiewandlung spielt die Dauer der Anregung im Verhältnis zur Schallausbreitung in der optischen Zone eine wesentliche Rolle (Unterabschnitt 2.2.2).

\subsubsection{Photothermische Energiewandlung}

Die photothermische Energiewandlung verläuft in zwei Schritten: Im ersten Schritt wird die Strahlungsenergie in den Rotations- und Schwingungsbanden der Wassermoleküle absorbiert. Im zweiten Schritt geben die angeregten Wassermoleküle ihre Energie durch Stöße an benachbarte Moleküle ab, womit sich deren kinetische Energie und damit die Temperatur im Wasser erhöht [5]. Beide Schritte sind in weniger als einer Nanosekunde abgeschlossen [80], so daß die Erwärmung der Wasserprobe als instantan bezüglich der betrachteten IR-Laserpulsdauern ( $>40 \mathrm{~ns}$ ) betrachtet werden kann.

Die Verteilung der Bestrahlungsstärke $I=\partial H / \partial t\left[\mathrm{~W} / \mathrm{mm}^{2}\right]$ über die Tiefe $z$ der Wasserprobe ist durch das Lambert-Beersche Absorptionsgesetz

$$
I(z)=I_{0} \exp (-\mu z)
$$

beschrieben, worin $I_{0}$ die Bestrahlungsstärke am Eingang der Wasserprobe ist und $\mu[1 / \mathrm{mm}]$ der Absorptionskoeffizient zur jeweiligen Laserwellenlänge. Die Bestrahlungsstärke erzeugt in einem Volumenelement $\mathrm{d} V=(\mathrm{d} x \mathrm{~d} y \mathrm{~d} z)$ in der Tiefe $z$ eine Leistungsdichte $Q\left[\mathrm{~W} / \mathrm{mm}^{3}\right]$ von der Größe [30]

$$
Q(z)=\left|\frac{\mathrm{d} I}{\mathrm{~d} z}\right|=I_{0} \mu \exp (-\mu z),
$$

die in einem Zeitintervall $\mathrm{d} t$ wiederum die Temperatur gemäß

$$
\mathrm{d} T(z)=\frac{1}{\rho C} Q(z) \mathrm{d} t
$$

erhöht. Hierbei ist $\rho\left[\mathrm{kg} / \mathrm{m}^{3}\right]$ die Dichte und $C[\mathrm{~J} /(\mathrm{kg} \mathrm{K})]$ die spezifische Wärme. Sie ist je nach Anregungsbedingungen bei konstantem Volumen $\left(C_{\mathrm{V}}\right)$ oder konstantem Druck $\left(C_{\mathrm{p}}\right)$ 
zu wählen: Für kurze Laserpulse, die eine thermoelastische Druckentwicklung nach sich ziehen, wird $C_{\mathrm{V}}$ eingesetzt ( Unterabschnitt 2.2.2), für lange Pulse mit vernachlässigbaren Druckänderungen $C_{\mathrm{p}}$.

Unter Vernachlässigung von Wärmeleitungseinflüssen wird die höchste Wassertemperatur im Maximum der Leistungsdichte bei $z=0$ erreicht. Für diesen Fall ergibt sich aus den Gl. (2.4) und (2.5) die einfache Beziehung

$$
\frac{\mathrm{d} T}{\mathrm{~d} t}=\frac{\mu}{\rho C} I_{0}(t)
$$

zwischen der Bestrahlungsstärke und der Änderung der Temperatur. Ist der Faktor $\mu /(\rho C)$ konstant, so berechnet sich die Temperatur zu einem gegebenen Zeitpunkt $t$ der Bestrahlung unmittelbar zu

$$
T(t)=T_{0}+\frac{\mu}{\rho C} \int_{-\infty}^{t} I_{0}(\tau) \mathrm{d} \tau .
$$

Weiter unten in diesem Unterabschnitt wird dargestellt, daß die Annahme $\mu /(\rho C)=$ const. für die betrachteten Laserwellenlängen nicht gültig ist, da der $\mathrm{Ab}$ sorptionskoeffizient $\mu$ eine ausgeprägte Temperaturabhängigkeit aufweist. Hierdurch wird aus Gl. (2.6) eine Differentialgleichung erster Ordnung, die durch „,Trennung der Variablen" entsprechend

$$
\int_{T_{0}}^{T} \frac{\mathrm{d} \tau}{\mu(\tau)}=\frac{1}{\rho C} \int_{-\infty}^{t} I_{0}(\tau) \mathrm{d} \tau
$$

gelöst werden kann, wenn eine Darstellung des Verlaufs von $\mu(T)$ vorliegt. Die Temperaturänderungen von Dichte und spezifischer Wärme sind gegenüber denen der Absorption weitgehend vernachlässigbar [8].

\section{Temperaturabhängigkeit der Absorption}

Das Absorptionsspektrum von Wasser im infraroten Spektralbereich ist durch ein kollektives Verhalten der Wassermoleküle geprägt. Dieses wird durch Wasserstoff-Brücken vermittelt, die sich zwischem den elektropositiven Wasserstoff- und den elektronegativen Sauerstoffatomen benachbarter Wassermoleküle bilden. Durch die Wasserstoff-Brücken wird das Absorptionsspektrum zum einen zu längeren Wellenlängen hin verschoben, bedingt durch den Einfluß der Brückenbindungen auf die Schwingungsfreiheitsgrade des 
einzelnen Wassermoleküls [80]. Darüber hinaus ist die Absorption der Moleküle im Kollektiv etwa zehnmal größer als die eines freien Moleküls [86]. Eine Erwärmung der Wasserprobe verringert die Bindungsstärke der Wasserstoff-Brücken wieder [50]. In der Folge schwächt zum einen die kollektive Absorption der Wassermoleküle ab und zum anderen verschiebt sich das Absorptionsspektrum zurück zu kürzeren Wellenlängen [31, 69]. Bei den Wellenlängen des Tm- und des Ho-Lasers $(2.01 \mu \mathrm{m}$ bzw. $2.1 \mu \mathrm{m})$ bewirkt diese Verschiebung eine zusätzliche Absorptionsminderung, da beide Wellenlängen auf der abfallenden Flanke des $1.94 \mu \mathrm{m}$-Absorptionsmaximums liegen (vgl. Abbildung 2.1). Die erwärmungsbedingte Abschwächung der Wasserstoff-Brückenbindungen erfolgt, wie die photothermische Energiewandlung, innerhalb von Pikosekunden [37]. Verglichen mit den betrachteten Laserpulsdauern können erwärmungsbedingte Änderungen der Absorption daher als instantan aufgefaßt werden.

Die Temperaturabhängigkeit der Absorption hat einen direkten Einfluß auf die Verteilung der Laserpulsenergie in der Wasserprobe, da die Laserstrahlung selbst die Probe erwärmt und somit die Absorptionsbedingungen für die nachfolgende Strahlung verändert. Für eine mit der Temperatur abnehmende Absorption wirkt sich dies wie ein „Ausbleichen“ der Wasserprobe aus, wodurch die Laserstrahlung tiefer in das Wasser eindringt, aber weniger hohe Temperaturen erzeugt als im Fall einer konstanten Absorption [47]. Dieser Effekt soll im folgenden für die betrachteten Tm- und Ho-Laserwellenlängen näher beziffert werden. Dazu werden zunächst die Temperaturverläufe der Absorptionen $\mu_{\mathrm{Tm}}(T)$, $\mu_{\mathrm{Ho}}(T)$ quantifiziert und anschließend die Differentialgleichung (2.8) nach der gesuchten Beziehung zwischen Temperatur und applizierter Bestrahlung ausgewertet.

Die Temperaturverläufe $\mu_{\mathrm{Tm}}(T), \mu_{\mathrm{Ho}}(T)$ sind in einer Arbeit von B. Lange, T. Brendel und G. Hüttmann [47] gezielt untersucht und für Temperaturen zwischen $20^{\circ} \mathrm{C}$ und $100^{\circ} \mathrm{C}$ mit hoher Genauigkeit vermessen worden. Durchgeführt wurde die Messung an einer Absorptionszelle variabler Weglänge. Diese bestand aus einer kleinen Quarzküvette, die mit Hilfe eines Mikroverstelltisches in eine größere Quarzküvette eingetaucht wurde. Die Transmission der Wasserschicht zwischen den Küvetten wurde mit einem kollimierten Probelaserstrahl der jeweiligen Wellenlänge ermittelt. Die Strahlprofile der Probelaser waren hierbei frei von Speckeln und die Bestrahlungen so weit reduziert, daß die laserinduzierte Temperaturerhöhung nicht mehr als $1{ }^{\circ} \mathrm{C}$ betrug. Zur Bestimmung jeweils eines Absorptionswertes wurde die Transmission für eine Reihe verschiedener Absorptionsweglängen vermessen. Durch semi-logarithmische Auftragung der Transmissionswerte über die Weglänge ergibt sich nach dem Lambert-Beer-Gesetz (2.3) ein linearer Verlauf, dessen Steigung der gesuchten Absorption entspricht. Dieses Verfahren hat gegenüber 
Messungen an einer festen Absorptionsstrecke [37] den wesentlichen Vorteil, daß die kritische Absolutbestimmung der Absorptionsweglänge entfällt. Lediglich die Weglängenänderungen müssen präzise bestimmt sein, was mit modernen Verstelltischen bis in den Sub-Mikrometerbereich möglich ist.

Mit dem dargestellten Verfahren wurde die Änderung der Absorption zwischen $20^{\circ} \mathrm{C}$ und $100^{\circ} \mathrm{C}$ in Schritten von $5^{\circ} \mathrm{C}$ ermittelt. Im Ergebnis ist für beide Laserwellenlängen ein streng linearer Abfall mit der Temperatur gefunden worden. Die Koeffizienten der zugehörigen linearen Regressionen sind in Tabelle 2.1 aufgeführt.

\begin{tabular}{|c|c|c|}
\hline & $\mathrm{a}[1 / \mathrm{mm}]$ & $\mathrm{b}\left[1 /{ }^{\circ} \mathrm{C} \mathrm{mm}\right]$ \\
\hline \hline $\mathrm{Tm}$ & $6.98 \pm 0.04$ & $-0.0259 \pm 3 \cdot 10^{-3}$ \\
\hline Ho & $3.39 \pm 0.01$ & $-0.0104 \pm 1 \cdot 10^{-3}$ \\
\hline
\end{tabular}

Tabelle 2.1: Koeffizienten zur linearen Regression der Absorption $\mu(T)=a+b T$ bei der Ho- und Tm-Laserwellenlänge [47]. Die Absorptionen bei $T=20^{\circ} \mathrm{C}$ betragen $\mu_{\mathrm{Tm}}=6.46 / \mathrm{mm}$ und $\mu_{\mathrm{Ho}}=3.18 / \mathrm{mm}$, als mittlere Absorptionen im Intervall $T \in[20,100]^{\circ} \mathrm{C}$ findet $\operatorname{man} \bar{\mu}_{\mathrm{Tm}}=5.43 / \mathrm{mm}$ und $\bar{\mu}_{\mathrm{Ho}}=2.77 / \mathrm{mm}$.

Durch den einfachen Linearverlauf $\mu(T)=a+b T$ läßt sich die in Gl. (2.8) beschriebene Temperaturänderung $\mathrm{d} T$ aus der Bestrahlungsstärke $I_{0}(t)$ analytisch bestimmen, was auf die folgende Zeitentwicklung der Temperatur führt:

$$
\left.T(t)\right|_{\mu}=\frac{1}{b}\left[\mu\left(T_{0}\right) \exp \left(\frac{b}{\rho C} \int_{-\infty}^{t} I_{0}(\tau) \mathrm{d} \tau\right)-a\right] .
$$

Für die Betrachtungen in dieser Arbeit sind insbesondere die erreichten Endtemperaturen von Interesse. Sie berechnen sich analog aus der applizierten Bestrahlung $H=\int_{-\infty}^{\infty} I(\tau) \mathrm{d} \tau$ $\mathrm{zu}$

$$
\left.T_{\mathrm{E}}(H)\right|_{\mu}=\frac{1}{b}\left[\mu\left(T_{0}\right) \exp \left(\frac{b H}{\rho C}\right)-a\right] .
$$

In Abbildung 2.5 ist dargestellt, welchen Verlauf die Funktion $\left.T_{\mathrm{E}}(H)\right|_{\mu}$ jeweils für die Anregung mit dem Tm- und dem Ho-Laser nimmt. Aufgetragen ist der Verlauf bis zu einer experimentell typischen Bestrahlung von $200 \mathrm{~mJ} / \mathrm{mm}^{2}$. Im Vergleich zum Temperaturverlauf bei konstant angenommener Absorption $\mu=\mu\left(20^{\circ} \mathrm{C}\right)$ verringert sich der Temperaturanstieg mit der Bestrahlung sichtlich. Umgekehrt erhöhen sich die Bestrahlungen, die beispielsweise zum Erreichen der Siedetemperatur $T=100^{\circ} \mathrm{C}$ benötigt werden. Für die 
Tm-Laseranregung beträgt die Erhöhung rund $19 \%$ (von $52 \mathrm{~mJ} / \mathrm{mm}^{2}$ auf $62 \mathrm{~mJ} / \mathrm{mm}^{2}$ ), für die Ho-Laseranregung sind es $17 \%$ (von $105 \mathrm{~mJ} / \mathrm{mm}^{2}$ auf $122 \mathrm{~mJ} / \mathrm{mm}^{2}$ ).
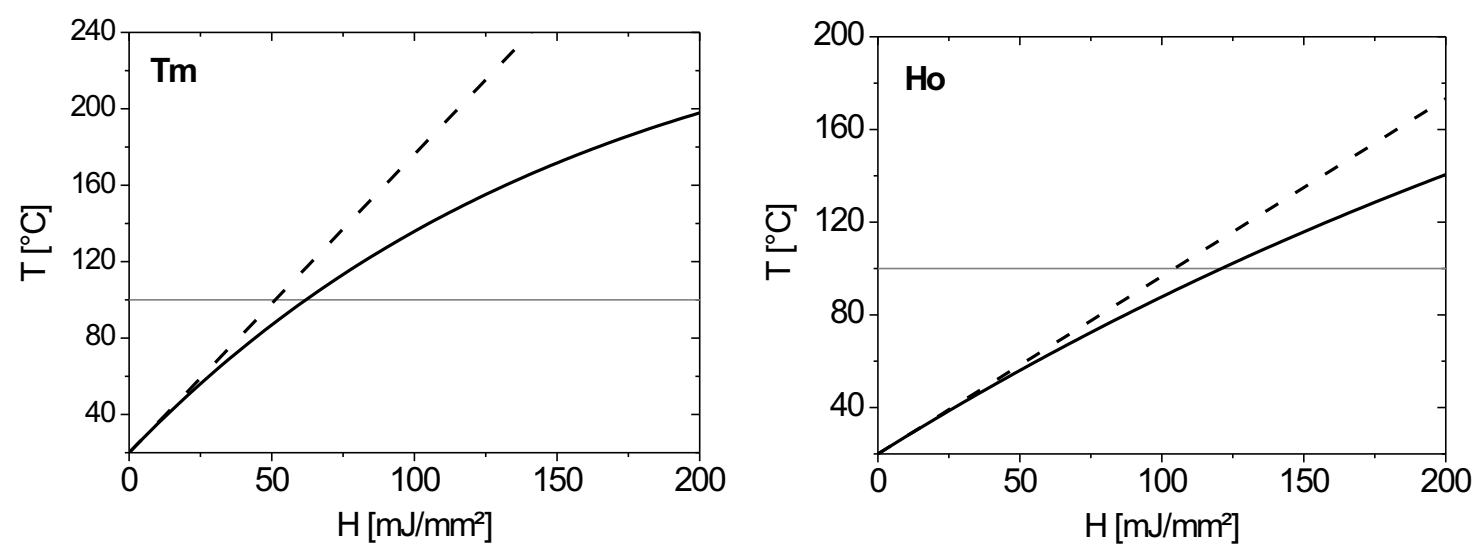

Abb. 2.5: Temperaturberechnung nach Gl. (2.10), unter Annahme der linearen Absorptionsverläufe $\mu_{\mathrm{Ho}}(T)$ bzw. $\mu_{\mathrm{Tm}}(T)$ gemäß Tabelle 2.1 (durchgezogene Linien). Die hellgraue Linie markiert die $100^{\circ} \mathrm{C}$-Grenze, oberhalb derer die Absorptionsverläufe extrapoliert sind. Zum Vergleich ist der Temperaturverlauf bei Annahme einer konstanten Absorption $\mu=\mu\left(20^{\circ} \mathrm{C}\right)$ eingezeichnet (gestrichelte Linien).

Um den dargestellten Bestrahlungsbereich rechnerisch abzudecken, muß der Linearverlauf der Absorptionen bereits weit extrapoliert werden, wie der frühe Schnittpunkt der Kurven mit der $100^{\circ} \mathrm{C}$-Linie verdeutlicht. Eine Bestimmung der Funktionen $\mu(T)$ oberhalb von $100^{\circ} \mathrm{C}$ mit dem oben beschriebenen Aufbau hätte den Einbau der Absorptionsstrecke in eine Druckzelle erfordert und damit eine Reihe technischer Mehraufwände nach sich gezogen [27]. Alternativ dazu läßt sich ein optisches Temperaturmeßverfahren einsetzen, das mit einem vergleichsweise geringen technischen Aufwand die experimentelle Überprüfung der berechneten Temperaturen erlaubt [8]. Grundlage für das Verfahren ist die gut dokumentierte Temperaturabhängigkeit der Brechzahl von Wasser [73], die durch Reflexionsmessungen an der Quarz-Wasser-Grenzfläche auf die erzielten Wassertemperaturen rückschließen läßt. Bis zu einer Wassertemperatur von ca. $180^{\circ} \mathrm{C}$ zeigten die gemessenen Temperaturen eine sehr gute Übereinstimmung zur Rechnung, so daß die Extrapolation bis zu dieser Temperatur als verläßlich eingestuft werden kann. Für Temperaturen, die darüber hinaus gehen, ist mit sukzessiven, nicht näher bestimmbaren Abweichungen zu rechnen. 


\section{Effekte durch Wärmeleitung}

Das laserinduzierte Temperaturprofil in der optischen Zone unterliegt einem stetigen Wärmeabfluß in das umgebende Probenvolumen und den angrenzenden Quarzkörper. Zur konservativen Abschätzung dieses axialen wie radialen Wärmeflusses sei beispielhaft eine Tm-Laserbestrahlung mit der (langen) Pulsdauer $t_{\mathrm{p}}=500 \mathrm{~ns}$ (FWHM), einem typischen Strahlradius $w=300 \mu \mathrm{m}$ und einer mittleren Absorption $\mu_{\mathrm{Tm}}=5.9 / \mathrm{mm}$ angenommen (Gaußscher oder fasergeführter Strahl). Es wird zunächst der radiale Wärmeabfluß betrachtet: Dieser läßt sich näherungsweise durch die thermische Relaxationszeit $\tau_{\mathrm{R}}$ für zylindergeometrische Temperaturprofile charakterisieren. Es ist [14]

$$
\tau_{\mathrm{R}}=\frac{d^{2}}{4 \gamma},
$$

mit dem Temperaturleitwert $\gamma=1.46 \cdot 10^{-7} \mathrm{~m}^{2} / \mathrm{s}$ für Wasser und dem Durchmesser $d$ des Zylinders. Für $d=2 w$ ergibt sich eine Relaxationszeit $\tau_{\mathrm{R}} \approx 0.6 \mathrm{~s}$. Diese liegt so deutlich über den betrachteten Anregungsdauern, daß die radiale Wärmeabfuhr für den Zeitraum der Bestrahlung vollkommen vernachlässigbar ist. Gleiches gilt für Strahlradien herab bis $\approx 25 \mu \mathrm{m}$, deren zugehörige Relaxationszeit $\tau_{\mathrm{R}} \geq 1 \mathrm{~ms}$ die betrachteten Anregungsdauern noch immer um drei Größenördnungen übertrifft.

Zur Berechnung des axialen Wärmeflusses wird ein 1-dimensionales Wärmeleitungsmodell verwendet, das in Anhang A.3 näher erläutert ist. In das Modell geht der zeitliche Pulsverlauf der Laseranregung ein und es wird der Wärmeabfluß in den Quarzkörper berücksichtigt. Mit der Quarz-Wasser-Grenzfläche bei $z=0$ und einem gaußförmigen Pulsverlauf (Abschnitt 2.1) ergibt sich das in Abbildung 2.6 dargestellte Profil der Temperaturerhöhung $\Theta=T-20^{\circ} \mathrm{C}$ über die Wassertiefe $z$. Um den Einfluß der Wärmeleitung zu verdeutlichen, ist das Profil auf die maximale Temperaturerhöhung $\Theta_{0}$ bei instantaner Energiedeponierung normiert. Das Temperaturprofil $\propto \exp (-\mu z)$ zur instantanen Energiedeponierung ist ebenfalls abgebildet. Aus dem Vergleich der beiden Kurven geht hervor, daß der Wärmeabfluß in den Quarzkörper das Temperaturprofil nur auf den ersten Mikrometern beeinflußt. Das Maximum der laserinduzierten Temperaturerhöung verschiebt sich von $z=0$ in eine Tiefe $z=1.6 \mu \mathrm{m}$, wo es noch $99 \%$ vom Maximum $\Theta_{0}$ ohne Wärmeleitung beträgt. Bezogen auf das gesamte Temperaturprofil, das sich über eine Eindringtiefe $\delta=1 / \mu$ von $\approx 180 \mu \mathrm{m}$ erstreckt, ist die axiale Wärmeabfuhr damit ebenfalls vernachlässigbar. 


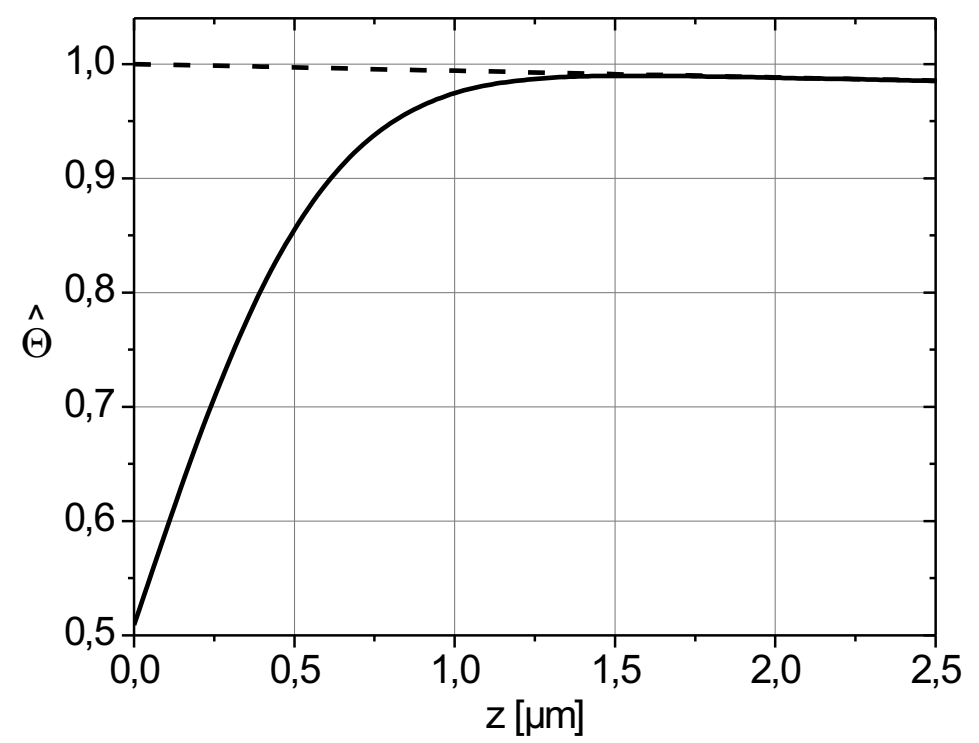

Abb. 2.6: Axialer Verlauf der normierten Temperaturerhöhung $\hat{\Theta}\left(t_{\mathrm{p}}, z\right)=\Theta\left(t_{\mathrm{p}}, z\right) / \Theta_{0}$, am Ende eines Laserpulses der Dauer $t_{\mathrm{p}}=500 \mathrm{~ns}$ (durchgezogene Linie) und bei instantaner Energiedeponierung (gestrichelte Linie).

\subsubsection{Photoakustische Energiewandlung}

Die Erhitzung einer Wasserprobe bewirkt zusätzlich eine Druckzunahme, wenn sie schneller erfolgt als die thermoelastische Expansion des Wassers. Ohne Expansion bleibt die Dichte $\rho$ konstant, so daß die Änderung des Druckes d $p$ unmittelbar von der Temperaturerhöhung $\mathrm{d} T$ abhängt: $\mathrm{d} p=(\partial p / \partial T)_{\rho} \mathrm{d} T$. Die positive Ableitung $(\partial p / \partial T)_{\rho}$ läßt sich durch die isobare Expansion $\beta_{\mathrm{p}}[1 / \mathrm{K}]$ und die isotherme Kompressibilität $\kappa_{\mathrm{T}}[1 / \mathrm{Pa}]$ darstellen [28]:

$$
\mathrm{d} p=\frac{\beta_{\mathrm{p}}}{\kappa_{\mathrm{T}}} \mathrm{d} T .
$$

Den Zusammenhang zwischen der Druckerhöhung und der applizierten Bestrahlung $\mathrm{d} H=I \mathrm{~d} t$ bringt die Verknüpfung von Gl. (2.12) mit der Relation (2.6), $\mathrm{d} T=\left(\mu / \rho C_{\mathrm{V}}\right) \mathrm{d} H$. Hierbei ist $C=C_{\mathrm{V}}$ gesetzt, da bei konstant angenommener Dichte auch das Volumen konstant ist. Durch Zusammenfassen aller thermodynamischen Materialparameter zum dimensionslosen Grüneisenkoeffizienten $\Gamma \equiv \beta_{\mathrm{p}} /\left(\kappa_{\mathrm{T}} \rho C_{\mathrm{V}}\right)$ folgt

$$
\mathrm{d} p=\mu \Gamma \mathrm{d} H
$$

In dieser Darstellung wird die Bedeutung des Grüneisenkoeffizienten als materialspezifisches Maß für die Effizienz der photoakustischen Energiewandlung deutlich. Wie alle 
Materialgrößen, aus denen er sich zusammensetzt, ist der Grüneisenkoeffizient temperaturabhängig (Unterabschnitt 3.2.1).

Gl. (2.13) beschreibt die differentielle Zunahme des Druckes in einem differentiellen Zeit- bzw. Bestrahlungsintervall $\mathrm{d} H$. Bei der Beschreibung der Druckentwicklung über die gesamte Dauer einer Laseranregung ist die thermoelastische Expansion des bestrahlten Probenvolumens nicht mehr vernachlässigbar. Die zugehörigen Dichteänderungen bringen zum Ausdruck, daß sich die Druckerhöhungen der optischen Zone als Schallwelle ausbreiten. Unter Voraussetzung eines linearen Zusammenhanges zwischen Druck und Dichte (vgl. Abschnitt 3.1) kann die Schallentwicklung durch die klassische Wellengleichung dargestellt werden. Durch Ergänzung um einen photoakustischen Quellterm mit einer Proportionalität analaog G1. (2.13) [30] vervollständigt sich die Beschreibung. Die resultierende inhomogene Wellengleichung wird entsprechend als photoakustische Wellengleichung bezeichnet [67]. Die Lösung der photoakustischen Wellengleichung ist Gegenstand von Kapitel 3.

Die differentiellen Druckerhöhungen in der optischen Zone akkumulieren sich zu einer umso höheren Gesamtamplitude, je schneller die Laseranregung gegenüber der Schallausbreitung verläuft. Eine instantane Energiedeponierung liefert die höchstmöglichen Amplituden, es wird in diesem Fall von einem optimalen ,,akustischen Einschluß“ (engl: stress confinement) gesprochen. Die akustischen Einschlußbedingungen zu einer zeitlich ausgedehnten Bestrahlung lassen sich in erster Näherung aus dem Verhältnis der Bestrahlungsdauer $t_{\mathrm{p}}$ zu der Zeit abschätzen, die eine Schallwelle der Geschwindigkeit $c_{0}$ zum Verlassen der optischen Zone benötigt [66]. Die Abmessungen der optischen Zone sind lateral durch den (Faser- oder Gaußschen) Strahldurchmesser $2 w$ und axial durch die optische Eindringtiefe $\delta=1 / \mu$ gegeben. Als charakteristische Länge $D$ sei der kleinere der beiden Werte genommen: $D \equiv \min (2 w, \delta)$. Die Größe

$$
M_{\mathrm{AE}} \equiv \frac{D}{t_{\mathrm{p}} c_{0}}
$$

ist dann ein $\mathrm{Maß}$ für den akustischen Einschluß, wobei große Werte von $M_{\mathrm{AE}}$ einen hohen Einschluß bedeuten. Zur Übersicht sind in Tabelle 2.2 die $M_{\mathrm{AE}}$-Werte zu verschiedenen Anregungsfällen aus der Literatur berechnet. Es handelt sich um die Arbeiten, die auch in Kapitel 5 zum Vergleich mit den Temperatur- und Druckmodellrechnungen dieser Arbeit herangezogen werden. Der akustische Einschluß ist am niedrigsten für einen Spike im Pulsprofil des freilaufenden Tm-Lasers (Abbildung 2.2), bedingt durch die verhältnismäßig lange Pulsdauer im Vergleich zu den gütegeschalteten Systemen. Da sich die Pulsenergie beim freilaufenden Laser (Gesamtpulsdauer: $300 \mu$ s) zudem auf eine Vielzahl 
von Spikes verteilt, ist die Energie eines Einzelspikes entsprechend gering. Eine nennenswerte thermoelastische Schallentwicklung ist deshalb nur bei Verwendung gütegeschalteter Lasersysteme zu beobachten.

\begin{tabular}{|l|c|c|c|c|c|}
\hline Laser & $\delta[\mu \mathrm{m}]$ & $2 w[\mu \mathrm{m}]$ & $t_{\mathrm{p}}[\mathrm{ns}]$ & $M_{\mathrm{AE}}$ & Referenz \\
\hline \hline Tm-Laser, gütegeschaltet & 180 & 660 & 300 & 0.4 & {$[8]$} \\
\hline Ho-Laser, gütegeschaltet & 310 & 420 & 80 & 2.6 & {$[8]$} \\
\hline Ho-Laser, gütegeschaltet & 310 & 300 & 45 & 4.5 & {$[24]$} \\
\hline Tm-Laser, freilaufend, Einzelspike & 180 & 120 & 1500 & 0.05 & {$[8]$} \\
\hline Nd:YAG, gütegeschaltet & 10 & 950 & 12 & 0.6 & {$[6]$} \\
\hline
\end{tabular}

Tabelle 2.2: Akustische Einschlußbedingungen zu verschiedenen Experimenten aus der Literatur. Die Schallgeschwindigkeit $c_{0}$ beträgt $1.48 \mu \mathrm{m} / \mathrm{ns}\left(\mathrm{T}=20^{\circ} \mathrm{C}\right)$.

In den Betrachtungen zum akustischen Einschluß ist unberücksichtigt, daß zu unterschiedlichen Abmessungen der optischen Zone unterschiedliche Pulsenergien benötigt werden, um vergleichbare Bestrahlungen zu erzielen. Ist die Pulsenergie im Experiment limitiert, so werden bei zunehmendem Strahlradius immer geringere Bestrahlungen und damit immer geringere Druckamplituden erzielt. Die Größe $M_{\mathrm{AE}}$ nimmt dagegen mit dem Strahlradius zu (bis zum Erreichen der jeweiligen Eindringtiefe $\delta$ ). Eine Optimierung der photoakustischen Energiewandlung allein durch Betrachtung der Größe $M_{\mathrm{AE}}$ ist daher nicht möglich.

\subsection{Blasenbildung durch Kavitation und Verdamp- fung}

Am Anfang jeder verdampfungs- oder kavitationsbedingten Blasenbildung stehen Phasengrenzen, die durch (sub-) mikroskopische Kavitäten, sog. „Nukleationskeime“, in der Flüssigkeit bereitgestellt werden [16, 43]. Ein Nukleationskeim, der sich frei im Wasser bewegt, geht mit der Zeit in Lösung oder treibt an die Wasseroberfläche [85]. Trotzdem finden sich in einer Wasserprobe, die über Stunden ruht, eine Vielzahl von Nukleationskeimen. Diese Beobachtung führte zu der Feststellung, daß die Keime in der Flüssigkeit stabilisiert sein müssen. Für die Stabilisierung kommen zwei Mechanismen in Betracht: Der eine beruht auf organischen Verunreinigungen in der Flüssigkeit, die sich als permeable Haut um den Keim legen und ihn so davor schützen, in Lösung zu gehen [49, 85]. 
Der Durchmesser eines derart stabilisierten Keims beträgt weniger als einen Mikrometer. Dies bewahrt ihn ferner vor einem schnellen Auftrieb an die Wasseroberfläche, da geringste Flüssigkeitsströmungen und die Brownsche Bewegung zu ständigen Richtungswechseln führen [49].

Der zweite Mechanismus besteht darin, daß sich der Nukleationskeim an die Oberfläche eines Schwebeteilchens oder einer Gefäßwandung heftet [13, 43, 49, 56, 85]. In Unterabschnitt 2.3.2 wird erläutert, wie sich der Keim in Spalten oder Rissen festsetzt, die er an der Oberfläche vorfindet. Dieser Stabilisierungsmechanismus steht in direktem Bezug zur fasergeführten IR-Laser-Anwendung. Dort ist es die Oberfläche der verwendeten Quarzfaser, die unmittelbar an die optische Zone grenzt und so potentiell Nukleationskeime für den Ablationsvorgang bereitstellt.

Die Bereitstellung von Phasengrenzen über stabiliserte Nukleationskeime wird als ,heterogene Nukleation“ bezeichnet [13]. Daneben können sich aus der laserinduzierten Erhitzung der Flüssigkeit weitere Keime bilden. Dieser als „homogene Nukleation“ bezeichnete Prozeß beruht auf statistischen Dichteschwankungen in der Flüssigkeit [16]. Er ist durch eine Nukleationsrate $J\left[\mathrm{~cm}^{-3} \mathrm{~s}^{-1}\right.$ ] geprägt, die nah am kritischen Punkt $T_{\text {krit }}=647 \mathrm{~K}$ $\left(374^{\circ} \mathrm{C}\right)$ steil ansteigt $[23,54]$. So beträgt die Nukleationsrate beim 0.89 -fachen von $T_{\text {krit }}$ [K] nur etwa $0.1 \mathrm{~cm}^{-3} \mathrm{~s}^{-1}$, beim 0.91 -fachen von $T_{\text {krit }}$ ist $J \approx 10^{21} \mathrm{~cm}^{-3} \mathrm{~s}^{-1}$ [58]. Als Folge dieses plötzlichen Anstieges verläuft der Phasenübergang explosiv. Dieser Mechanismus spielt insbesondere bei Anwendungen des gepulsten Erbium-Lasers eine Rolle [81], wo aufgrund der hohen Wasserabsorption $\mu_{\mathrm{Er}} \simeq 1200 / \mathrm{mm}$ bereits bei mittleren Pulsenergien hohe Temperaturen erreicht werden. Für die Tm- und Ho-Laserablation ist dagegen die heterogene Nukleation der vorrangige Auslösemechanismus, da aufgrund der Absorptionen von deutlich unter 10/mm (Unterabschnitt 2.2.1) Temperaturen von typischerweise $0.8 \times T_{\text {krit }}$, entsprechend $250^{\circ} \mathrm{C}$, nicht überschritten werden [8].

\subsubsection{Blasenentstehung aus einem Nukleationskeim}

Ausgangspunkt der Betrachtungen sei ein sphärischer Nukleationskeim, der sich im statischen Gleichgewicht befinde (Abbildung 2.7). Unter Vernachlässigung des Dampfdruckes der umgebenden Flüssigkeit $\left(0.02\right.$ bar für Wasser bei $20^{\circ} \mathrm{C}$ ) beträgt der Gasdruck $p_{\mathrm{i}}$ im inneren des Keims

$$
p_{\mathrm{i}}=p_{0}+\frac{2 \sigma}{R_{0}} \equiv p_{0}+p_{\sigma} .
$$

Darin ist $p_{0}$ der hydrostatische Ruhedruck, $\sigma[\mathrm{N} / \mathrm{m}]$ die Oberflächenspannung und $R_{0}$ 


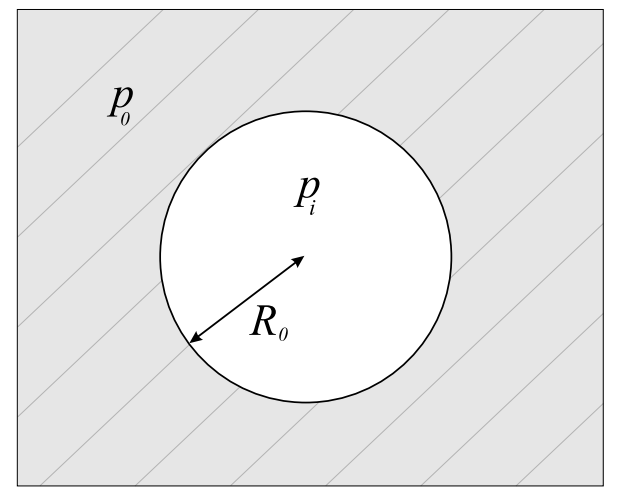

Abb. 2.7: Sphärischer Nukleationskeim mit Radius $R_{0}$ und Innendruck $p_{\mathrm{i}}$, der von einer Flüssigkeit mit hydrostatischem Ruhedruck $p_{0}$ umgeben ist.

der Gleichgewichtsradius des Keims. Aufgrund des nach innen gerichteten Oberflächenspannungsdruckes $\left(p_{\sigma}>0\right)$ ist der Gasdruck im Keim stets größer als der umgebende Flüssigkeitsdruck. Dieser Druckunterschied treibt den Gasinhalt des Keims zunehmend in Lösung [49], die Lebensdauer des Keims ist somit begrenzt.

Zum Verständnis der Blasenentstehung ist das einfache Gleichgewichtsmodell trotzdem dienlich und soll nun auf die beiden Vorgänge der Kavitation und Verdampfung angewendet werden:

\section{Verdampfung}

Für einen verdampfungsbedingten Phasenübergang sind verschiedene Energiebeiträge aufzubringen: Zunächst derjenige zum Erreichen der Siedetemperatur, dann die ,innere Energie“ zur Überwindung der intermolekularen Anziehungskräfte und zuletzt die ,äußere Energie“ zum Leisten der Volumenarbeit $p \Delta V$ gegen den Umgebungsdruck. Innere und äußere Energie werden zur sog. Verdampfungsenthalpie zusammengefaßt [55]. Der größte Energiebeitrag ist für die innere Energie aufzubringen. Er beträgt $2249 \mathrm{~kJ} / \mathrm{kg}$ (bei $100^{\circ} \mathrm{C}$ ), gegenüber $170 \mathrm{~kJ} / \mathrm{kg}$ für die Volumenarbeit (bei Normaldruck) und $334 \mathrm{~kJ} / \mathrm{kg}$ für die Erwärmung des Wassers von $20^{\circ} \mathrm{C}$ auf $100^{\circ} \mathrm{C}$ [26]. Ist der Siedepunkt erreicht, so trägt die Verdampfungsenthalpie als „latente Wärme“ ausschließlich zum Phasenübergang bei und die Temperatur bleibt bis zum Ende des Vorganges konstant.

Bei einer Verdampfung an der Phasengrenze des Nukleationskeimes stößt die flüssige Phase auf eine Dampf-Phase mit erhöhten Innendruck $p_{\mathrm{i}}$. Je nach Größe von $p_{\mathrm{i}}$ ergibt sich eine andere Siedetemperatur. Der hierfür ursächliche Oberflächenspannungsdruck $p_{\sigma}$ ist 
eine Funktion des Keimradius und der Temperatur, infolge einer temperaturabhängigen Oberflächenspannung $\sigma(T)$. Die Oberflächenspannung nimmt kontinuierlich mit $T$ ab und erreicht am kritischen Punkt den Wert Null. Zwischen $100^{\circ} \mathrm{C}$ und $250^{\circ} \mathrm{C}$ läßt sie sich in guter Näherung durch eine Gerade der Form

$$
\sigma(T)=79.3-0.2133 T
$$

beschreiben [13], mit den Einheiten $[\mathrm{mN} / \mathrm{m}]$ für $\sigma$ und $\left[{ }^{\circ} \mathrm{C}\right]$ für $T$. Zusammen mit der Dampfdruckkurve des Wassers (Abbildung 2.8) kann nun aus der Gleichgewichtsbedingung (2.15) die Siedetemperatur als Funktion des Keimradius bestimmt werden. Der zu-

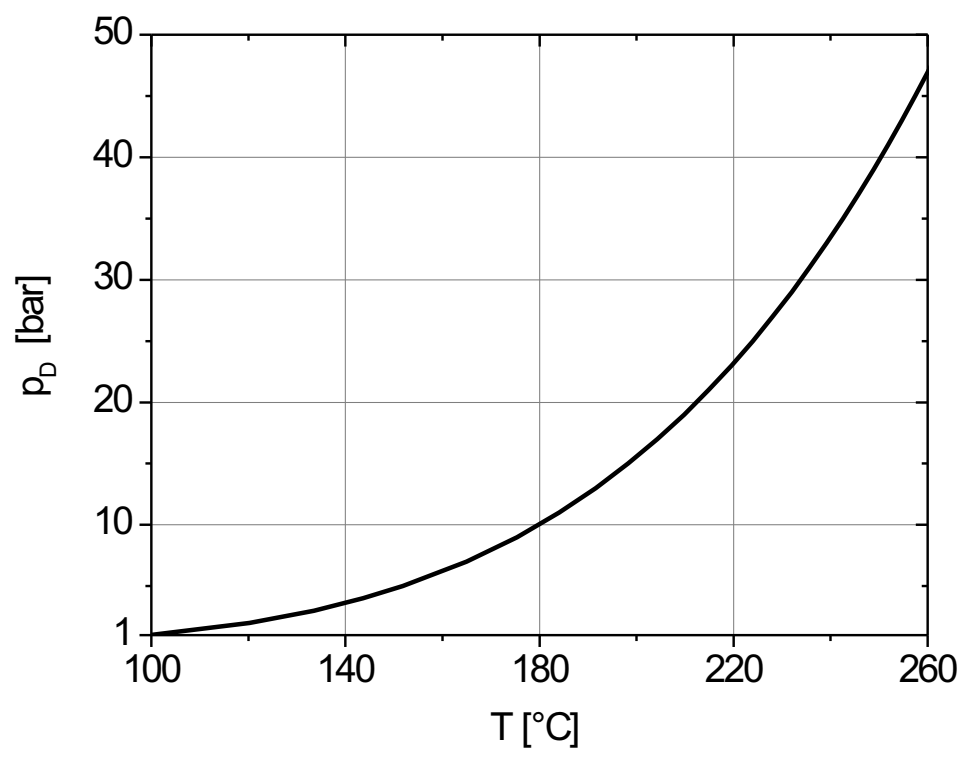

Abb. 2.8: Dampfdruckkurve von Wasser [34].

gehörige Verlauf ist in Abbildung 2.9 aufgetragen. Er zeigt, daß für Keimradiengrößen bis zu einem Mikrometer die Siedetemperaturen deutlich oberhalb von $100^{\circ} \mathrm{C}$ liegen. Sollte die Wasserprobe keine größeren Keime aufweisen (etwa durch intensive Filterung), so kann es folglich zu einer Überhitzung der Wasserprobe kommen. Diese kann aber auch einsetzen, falls die Keimzahldichte zu gering ist [54] oder die Energiedeponierung bezüglich der Wachstumsraten der Keime zu schnell verläuft [79].

\section{Kavitation}

In ungefilterten Flüssigkeiten ist bei statischer Zugbelastung bereits ab $p=-1$ bar mit Kavitation zu rechnen [35]. Bei dynamischer Belastung, etwa durch Zugwellen nach 


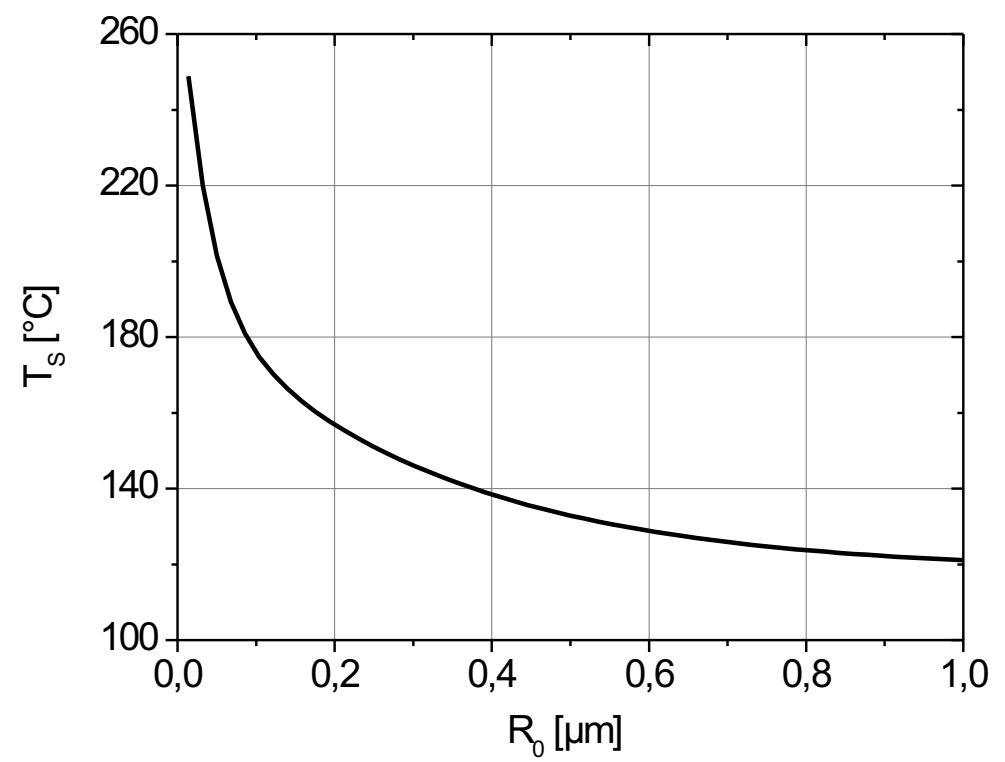

Abb. 2.9: Siedetemperatur als Funktion des Keimradius, berechnet unter Berücksichtigung der Temperaturabhängigkeit der Oberflächenspannung.

Unterwasserexplosionen oder durch gepulste Laseranregung, liegt die Kavitationsschwelle bei -5 bis -10 bar $[49,63]$. Zum Verständnis des Kavitationsvorganges wird nun betrachtet, wie der sphärische Nukleationskeim auf eine Druckminderung in der Flüssigkeit reagiert. Es sei zunächst angenommen, daß sich der Druck nur langsam verringert, so daß sich der Keim beständig im Gleichgewicht befindet. Eine solche quasi-statische Druckminderung bewirkt eine isotherme Vergrößerung des Keims und damit eine Abnahme des Gasdruckes gemäß [85]

$$
p_{\mathrm{i}}=\left(p_{0}+\frac{2 \sigma}{R_{0}}\right)\left(\frac{R_{0}}{R}\right)^{3},
$$

mit dem Gleichgewichtsradius $R$ zum verminderten Flüssigkeitsdruck $p_{\mathrm{F}}$. Da $p_{\mathrm{i}}$ nicht negativ werden kann, läßt sich die Druckdifferenz zwischen Gas und Flüssigkeit durch eine Zugbelastung $\left(p_{\mathrm{F}}<0\right)$ beliebig steigern. Der Oberflächenspannungsdruck $p_{\sigma}$, der die Druckdifferenz im statischen Gleichgewicht kompensiert, nimmt dagegen mit wachsendem Keimradius ab. Folglich muß es einen Schwellenwert $p_{\text {в }}$ für die Zugbelastung geben, bei dessen Überschreiten der Keim aus dem Gleichgewicht gedrängt wird und explosionsartig zu wachsen beginnt - die Kavitation setzt ein. 
Die Größe $p_{\text {в }}$ wird als „Blake“-Schwelle bezeichnet [49]. Ihr Betrag läßt sich ermitteln, indem aus der Gleichgewichtsbedingung $p_{\mathrm{i}}=p_{\mathrm{F}}+p_{\sigma}$ eine Abhängigkeit des Flüssigkeitsdruckes vom Keimradius formuliert wird:

$$
\left.p_{\mathrm{F}}(R)\right|_{R_{0}}=p_{\mathrm{i}}-p_{\sigma}=\left(p_{0}+\frac{2 \sigma}{R_{0}}\right)\left(\frac{R_{0}}{R}\right)^{3}-\frac{2 \sigma}{R} .
$$

Da diese Funktion beschreibt, welchen Wert $p_{\mathrm{F}}$ für einen gegebenen Keim vom Radius

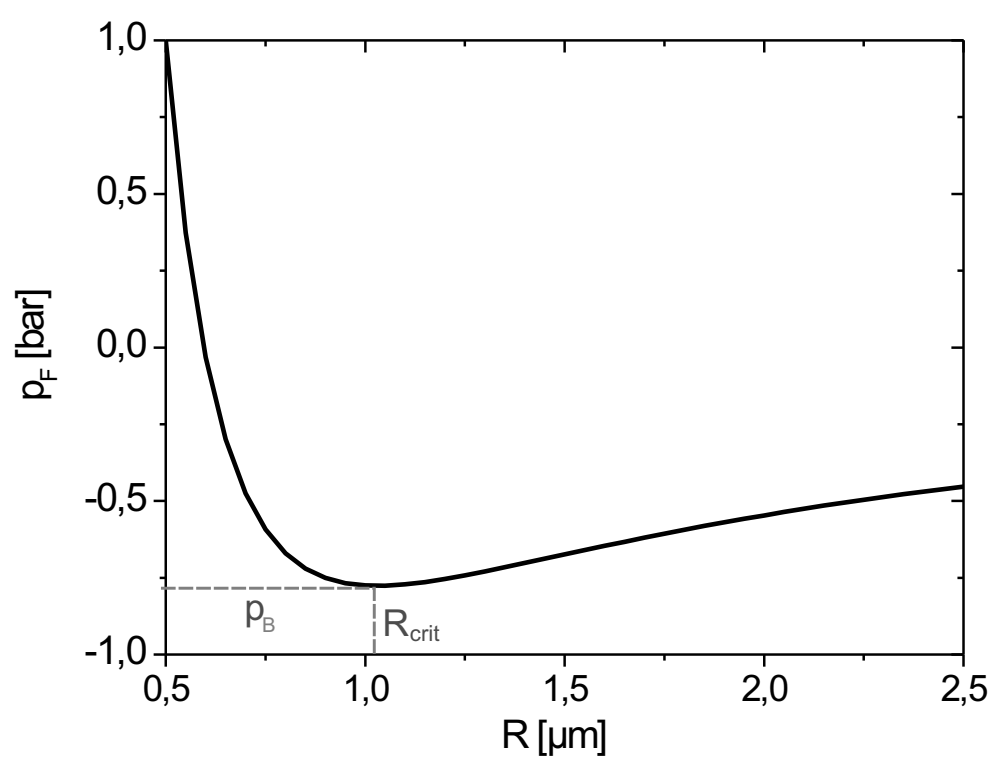

Abb. 2.10: Exemplarischer Verlauf von $p_{\mathrm{F}}(R)$, berechnet aus Gl. (2.18) für einen Ruheradius $R_{0}=0.5 \mu \mathrm{m}$, einen Ruhedruck $p_{0}=1$ bar und eine Oberflächenspannung $\sigma\left(100^{\circ} \mathrm{C}\right)=$ $59 \mathrm{mN} / \mathrm{m}$.

$R$ annehmen muß, damit sich dieser im statischen Gleichgewicht befindet, entspricht die Blake-Schwelle gerade dem kleinstmöglichen Wert, den $p_{\mathrm{F}}$ annehmen kann. Dieses Minimum bestimmt sich aus der Forderung $\partial p_{\mathrm{F}} / \partial R=0 \mathrm{zu}[49]$ :

$$
p_{\mathrm{B}}=\frac{4 \sigma}{3 R_{\text {krit }}}, \quad \text { mit } \quad R_{\text {krit }}=\sqrt{\frac{3 R_{0}^{3}}{2 \sigma}\left(p_{0}+\frac{2 \sigma}{R_{0}}\right)}
$$

Hierbei ist $R_{\text {krit }}$ der sogenannte kritische Radius, bei dem das Minimum erreicht wird (Abbildung 2.10). Für typische Keimgrößen im Bereich um einen Mikrometer liegt die Blake-Schwelle in der Größenordnung von $p=-1$ bar und bestätigt damit die experimentell gefundenen Kavitationsschwellen [35].

Mit der Annahme einer quasi-statischen Druckänderung sind dynamische Einflüsse wie die Trägheit oder Viskosität der Flüssigkeit vernachlässigt worden. Um diese einzubinden, 
sind weiterführende Modelle erforderlich [49, 85], die hier jedoch nicht diskutiert werden sollen. Für die weitere Diskussion in dieser Arbeit genügt ein prinzipielles Verständnis des Kavitationsvorganges.

\subsubsection{Heterogene Nukleation an einem Oberflächendefekt}

Die Form eines Nukleationskeimes an einer festen Oberfläche ist abhängig von den herrschenden Grenzflächenspannungen zwischen den beteiligten Medien Gas/Dampf, Flüssigkeit und Festkörper [13]. Aus dem Kräftegleichgewicht dieser Spannungen bildet sich ein materialspezifischer Kontaktwinkel heraus, wie er in Abbildung 2.11 a beispielhaft dargestellt ist. Analog zum sphärischen Nukleationskeim hat auch der anhaftende Keim eine konvexe Form und wird damit ebenso in Lösung getrieben, da die nach innen gerichtete Oberflächenspannung den Gasdruck erhöht. Eine glatte Oberfläche kann den Keim also nicht stabilisieren. Die Abbildung 2.11 b zeigt eine Oberfläche, die einen (idealisiert) konusförmigen Defekt aufweist. Wenn der Keim den gesamten Defekt auffüllt und wie dargestellt darüber hinaus ragt, ist er genauso instabil wie der Keim an der glatten Oberfläche. Wenn er aber im Inneren des Defektes sitzt (Abbildung $2.11 \mathrm{c}$ ), so
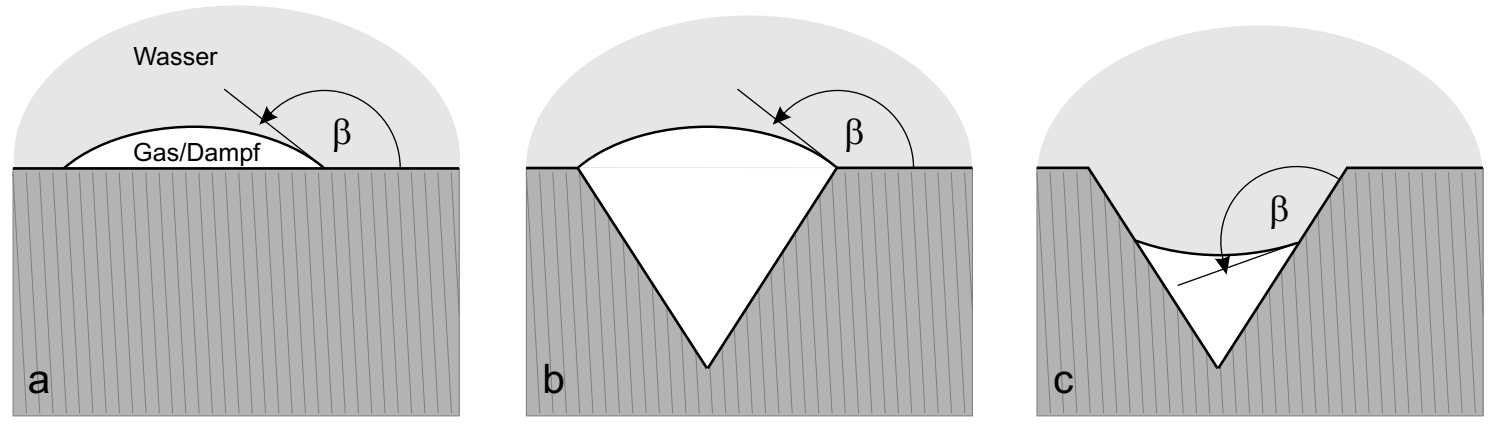

Abb. 2.11: a) Nukleationskeim mit Kontaktwinkel $\beta$ an einer festen Oberfläche b) Nukleationskeim in Oberflächenspalte c) Stabilisierter Nukleationskeim in Oberflächenspalte. (In Anlehnung an T. G. Leighton [49])

ergibt sich eine andere Situtation: Zwar ist der Kontaktwinkel derselbe wie in den Fällen $a$ und $b$, doch führt die beiderseitige Wandnähe jetzt zu einer konkaven Keimkrümmung. Entsprechend ist der Oberflächenspannungsdruck nach außen gerichtet und der Gasdruck im Keim niedriger als der umgebende Flüssigkeitsdruck. Der Gasinhalt wird hierdurch gehalten, der Keim stabilisiert und zugleich die Aktivierungsschwelle des Keims herabgesetzt. Bei einem Herauswachsen aus dem Defekt wechselt die Keimkrümmung jedoch 
zurück in die konvexe Form, so daß ein Fortschreiten des Keimwachstums wieder mit höheren Aktivierungsschwellen verbunden ist.

Der dargestellte Oberflächendefekt stellt eine starke Idealisierung dar, beschreibt aber zutreffend das Prinzip der Stabilisierung. Reale Oberflächendefekte sind irregulär, so daß die Krümmungsradien und damit die Aktivierungsschwellen der Keime stark variieren. Zudem ist nicht jeder Defekt in der Lage, einen Keim einzufangen. Hierzu bedarf es einer geeigneten Defektgeometrie, die das Eindringen einer vorbeilaufenden Flüssigkeitsfront verhindert und damit eine Gaslücke im Defekt entstehen läßt [13].

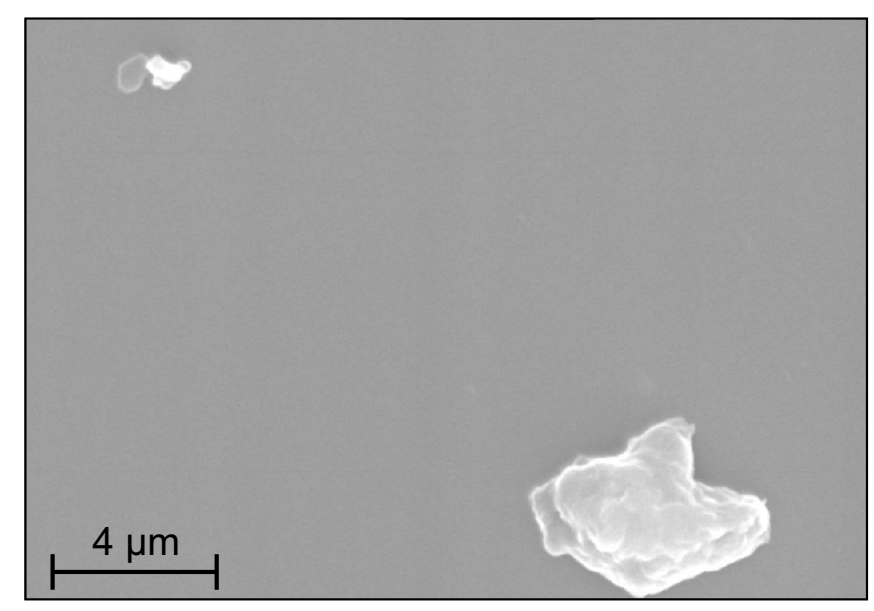

Abb. 2.12: REM-Aufnahme einer gereinigten, synthetischen Quarzoberfläche optischer Güte. Eine Spektralanalyse mit dem Elektronenstrahl zeigte, daß die sichtbaren Partikel wie das Substrat aus Silizium bestehen. Es handelt sich daher vermutlich um Partikelreste vom Poliervorgang. Man beachte die rauhe Oberflächenstruktur der Partikel, die zahlreiche Ansatzpunkte für Nukleationskeime bietet (Abbildung mit freundlicher Genehmigung der FH Lübeck).

Aufgrund der komplexen Verhältnisse bereits am Einzeldefekt ist es in der Praxis unmöglich, die Nukleationsbedingungen eines gesamten Experimentes zu definieren. Zwar läßt sich durch sorgfältige Präparation der untersuchten Probe (Entgasung, Filterung) und der angrenzenden Quarzoberfläche die Anzahl an potentiellen Nukleationskeimen deutlich verringern $[16,60]$. Trotzdem sind Restverschmutzungen insbesondere an Oberflächen nicht zu verhindern $[2,78]$. Des weiteren können Quarzoberflächen von nominell gleicher optischer Güte völlig unterschiedliche Defektstrukturen aufweisen, wie etwa Risse, Krater oder Partikelreste des Poliervorganges (vgl. Abbildung 2.12). Die Ausdehnung der Defekte bleibt hierbei nicht auf die Oberfläche beschränkt, sondern kann 
tief in das Substrat reichen, ohne daß dies an der Oberfläche zu erkennen wäre [42, 77, 83]. Ein Keim, der sich an einem solchen Defekt stabilisiert, hat eine deutlich längere Lebensdauer, da sich ihm ein vergrößertes Gasreservoir bietet [60, 61].

Die indiviudell unterschiedlichen Nukleationsbedingungen haben zur Folge, daß sich zu jedem Experiment ebenso individuelle Verdampfungs- und Kavitationsschwellen einstellen: Je nach Anzahl und Größenverteilung der Nukleationskeime müssen unterschiedliche Energien aufgewendet werden, um aus den Keimen Blasen entstehen zu lassen. In Experimenten zur gepulsten Tm-Laseranregung von entgasten Reinstwasserproben wurde dieser Effekt von Brendel et al. quantifiziert [7], indem die Ablationsschwellen bei Wechsel der Quarzoberflächen ermittelt wurden. Aus 25 Versuchen mit synthetischen Quarzoberflächen gleicher optischer Güte ergab sich hierbei ein Faktor von 3.7 zwischen der geringsten und der höchsten Schwelle. Im Literatur-Vergleich von Arbeiten zur gepulsten IR-Laser-Ablation ist dieser Effekt unbedingt zu berücksichtigen. 


\section{Darstellung der thermoelastischen Schallentwicklung}

\section{1. Übersicht}

Die thermoelastische Schallanregung durch einen Gaußschen Laserstrahl ist für Anwendungen aus der optoakustischen Tomographie und Spektroskopie vielfach theoretisch beschrieben worden [20, 30, 38, 39, 40, 41, 45, 51, 53]. In diesen Anwendungen erfolgt die Schalldetektion in großem Abstand zur Schallquelle (einige $\mathrm{mm}$ bis $\mathrm{cm}$ ) und damit im akustischen Fernfeld. Für eine initial gaußförmige Druckerhöhung wird das akustische Fernfeld im Abstand

$$
z \gg \frac{\mu w^{2}}{4}
$$

zum Lasereintritt in die Probe erreicht [38]. Mit den Absorptionswerten zur Ho- und TmLaserwellenlänge und einem typischen Strahlradius $w=300 \mu \mathrm{m}$ ergeben sich Abstände von $z \gg 70 \mu \mathrm{m}$. Die höchsten Druck- und Temperaturänderungen werden dagegen in der Eintrittsebene $z=0$, dem Ort der höchsten Bestrahlung, erreicht. Die relevanten thermodynamischen Zustandsänderungen sind damit im akustischen Nahfeld und nicht im Fernfeld zu erwarten.

Zum akustischen Nahfeld finden sich in der Literatur nur wenige Untersuchungen. Die geringe Zahl an Arbeiten mag dadurch begründet sein, daß im Fernfeld zahlreiche Näherungsannahmen zulässig sind, so daß die thermoelastische Schallentwicklung dort erheblich leichter zu berechnen ist. So sind es im wesentlichen die Arbeiten von G. Paltauf et al. $[25,67,68]$ zur fasergeführten Laseranregung, sowie die grundlegende Arbeit von 
V. Ristic [72] zum Schallfeld des Kolbenstrahlers, in denen auch das Nahfeld modellhaft untersucht wird.

Im Rahmen der vorliegenden Arbeit wurden weiterführende Modelle zur Schallentwicklung im akustischen Nahfeld entwickelt, um eine vertiefte Vorstellung von der thermodynamischen Zustandsentwicklung in der angeregten Wasserprobe zu gewinnen. Betrachtet wurden dabei die Bestrahlungsgeometrie zum Gaußschen wie zum fasergeführten Laserstrahl. Mit den Überlegungen aus Unterabschnitt 2.2.2 diente die photoakustische Wellengleichung als Ausgangspunkt für die Modellierung. Da der photoakustische Quellterm den Charakter eines Potentials für die Bewegung der Flüssigkeitsteilchen hat, wird die Gleichung üblicherweise für das Skalarpotential $\Phi(\vec{x}, t)\left[\mathrm{m}^{2} / \mathrm{s}\right]$ der Schallschnelle $\vec{v}=\nabla \Phi$ angegeben. Hierfür lautet sie [30]

$$
\nabla^{2} \Phi-\frac{1}{c_{0}^{2}} \frac{\partial^{2} \Phi}{\partial t^{2}}=\frac{\mu \Gamma}{\rho_{0} c_{0}^{2}} I(\vec{x}, t) .
$$

Darin ist $\rho_{0}$ die Ruhedichte mit zugehöriger Schallgeschwindigkeit $c_{0}, \mu$ die Absorption, $I(\vec{x}, t)=\partial H(\vec{x}, t) / \partial t$ die Bestrahlungsstärke zur einfallenden Bestrahlung $H$ und $\Gamma$ der Grüneisenkoeffizient. Der Druck ergibt sich aus $\Phi$ gemäß [46]

$$
p=-\rho_{0} \frac{\partial \Phi}{\partial t}
$$

Bei der Lösungsbehandlung der photoakustischen Wellengleichung ist es wesentlich, einen 3-dimensionalen Lösungsansatz zu wählen, um Beugungseffekte bei der Schallausbreitung berücksichtigen zu können [25]. Ein zentrales Beugungsphänomen ist die Entstehung von Zuganteilen aus der ursprünglich rein positiven Druckänderung. Die vielfach beschriebene Zugwellenbildung durch Reflexion an einer freien Grenzfläche (z.B. Wasser-Luft [17, 62, 64]) kommt für die minimal-invasive Anwendung nicht in Betracht, da die vorliegende Quarz-Wasser-Grenzfläche schallhart ist und einen positiven Reflexionskoeffizienten hat. Um die Beugungseffekte berücksichtigen zu können, wird in dieser Arbeit ein 3-dimensionales Lösungsintegral aus der Potentialtheorie verwendet. Aufgrund seiner Komplexität ist dieses Integral in seiner Ausgangsform aber wenig praktikabel. Durch Separation von Raum- und Zeitabhängigkeit der Laseranregung läßt sich das Integrationsgebiet jedoch um eine Dimension reduzieren, wie in Abschnitt 3.2 dargestellt ist. Im Ergebnis führt die Separation auf die bekannte „Poisson'sche Formel“ der Akustik zurück [46].

Auch wenn die Poisson'sche Formel gegenüber dem ursprünglichen Lösungsintegral eine Verbesserung darstellt, ist ihre Berechnung noch immer aufwändig. Die Berechnung 
vereinfacht sich jedoch für Punkte, die auf der Strahlachse liegen. Dort bewirkt die Radialsymmetrie eines Faser- oder Gaußstrahlprofils, daß sich die Integration auf eine verbleibende Raum-Dimension verringert. Im Fall des fasergeführten Lasers läßt sich das verbleibende Integral unter Annahme eines ideal rechteckigen Strahlprofils analytisch lösen und eine entsprechende Lösungsfunktion zur Schallentwicklung auf der Strahlachse angeben [67]. Die unstetigen Kanten eines Rechtprofils beschreiben die experimentellen Bestrahlungsverhältnisse an einem Faserende jedoch ungenügend. Erweiterungen des Profils um stetige, meist gaußförmige Flanken bewirken, daß das verbleibende Integral wiederum numerisch behandelt werden muß $[6,67]$.

Im Fall der Anregung durch einen Gaußschen Laserstrahl behandeln G. Paltauf [67] et al. die Poisson'sche Formel vollständig numerisch. In den Berechnungen zu dieser Arbeit hat sich dagegen gezeigt, daß es auf der Strahlachse eine analytische Lösung gibt (Abschnitt 3.3). Nach Kenntnisstand des Verfassers kann damit erstmalig zur Anregung durch einen Gaußschen Laserstrahl eine Lösungsfunktion angegeben werden, die die Schallentwicklung geschlossen im Nah- und Fernfeld beschreibt. Hierdurch eröffnet sich die Möglichkeit, die akustischen Einflüsse in der thermodynamischen Zustandsentwicklung der optischen Zone eingehend zu analysieren. Die Ergebnisse dieser Analyse werden in Kapitel 4 vorgestellt.

\section{Linearität der Schallausbreitung}

Die Wellengleichung (3.2) beschreibt ausschließlich lineare Vorgänge der Schallausbreitung, d.h. sie verliert ihre Gültigkeit im Fall hoher Druckamplituden und bei der Ausbildung von Stoßwellen. Der Begriff „linear“ bezieht sich hierbei auf die linearisierte Form der Gleichungen, aus denen die Wellengleichung hergeleitet wird. Dieses sind die Eulerund Kontinuitätsgleichung sowie die Zustandsgleichung, die Druck und Dichte in der Schallwelle miteinander verknüpft [46]. Im folgenden wird die Linearisierung exemplarisch für die Zustandsgleichung dargestellt:

Die Ausbreitung einer Schallwelle verläuft adiabatisch, d.h. ohne Änderung der Entropie $S$, da aufgrund der Geschwindigkeit des Vorganges kein Wärmeaustausch zwischen den Flüssigkeitsteilchen stattfindet. Druck und Dichte in der Schallwelle hängen entsprechend über eine adiabate Zustandsgleichung wie z.B. die bekannte Tait-Gleichung zusammen 
$[4,71]$. Für eine allgemeine Darstellung kann die Zustandsgleichung in eine Taylorreihe

$$
p(\rho)=p_{0}+\left.\frac{\partial p}{\partial \rho}\right|_{S, \rho_{0}}\left(\rho-\rho_{0}\right)+\left.\frac{1}{2} \frac{\partial^{2} p}{\partial \rho^{2}}\right|_{S, \rho_{0}}\left(\rho-\rho_{0}\right)^{2}+\ldots
$$

um die Ruhedichte $\rho_{0}$ entwickelt werden, wobei die Entwicklung bis zur quadratischen Ordnung zumeist ausreichend ist [4]. Die Schallgeschwindigkeit $c$ berechnet sich hieraus definitionsgemäß zu [46]

$$
c^{2}=\left.\frac{\partial p}{\partial \rho}\right|_{S, \rho}
$$

Die Reihendarstellung läßt sich weiter vereinfachen, indem die reduzierte Dichte $\hat{\rho}=\left(\rho-\rho_{0}\right) / \rho_{0}$ eingeführt wird, was auf

$$
p(\hat{\rho}) \simeq p_{0}+A \hat{\rho}+\frac{B}{2} \hat{\rho}^{2}
$$

führt. Die Koeffizienten $A$ und $B$ sind durch Gl. (3.4) eindeutig festgelegt. So ist insbesondere

$$
A=\left.\rho_{0} \frac{\partial p}{\partial \rho}\right|_{S, \rho_{0}}=\rho_{0} c_{0}^{2}
$$

worin $c_{0}$ die Schallgeschwindigkeit zu finiten Auslenkungen um $\rho_{0}$ ist. Für Wasser berechnet sich $A$ bei Raumtemperatur zu $2.186 \cdot 10^{4}$ bar und es ist $B / A=5.0$ [4, 34]. Das Verhältnis $B / A$ des linearen zum quadratischen Reihenkoeffizienten wird als akustischer Nichtlinearitätsparameter bezeichnet [15, 21].

Die Darstellung der adiabaten Zustandsgleichung als Potenzreihe der Form (3.6) geht auf R. T. Beyer zurück [3, 4]. Ihr großer Nutzen liegt darin, daß sie den Druckverlauf über einen weiten Dichtebereich wiedergibt, obwohl zur Bestimmung der Koeffizienten $A$ und $B$ nur Messungen nahe der Ruhedichte durchgeführt werden (gemäß der Entwicklung der Taylorreihe um $\rho_{0}$ ) [15]. Zudem ist die Reihendarstellung vorteilhaft für die Untersuchung der Frage, ob die Schallausbreitung linear oder nichtlinear erfolgt [46]. Eine nichtlineare Schallausbreitung wird durch die Dichteabhängigkeit der Schallgeschwindigkeit vermittelt. Diese hat mit Gl. (3.5) und (3.6) die Form

$$
c^{2}(\hat{\rho}) \simeq c_{0}^{2}\left(1+\frac{B}{A} \hat{\rho}\right)
$$

und besagt eine Zunahme der Schallgeschwindigkeit mit der Dichte, da $B / A$ positiv ist. Hierdurch besteht die Möglichkeit, daß sich die Schallwelle im Verlauf der Ausbreitung so weit aufsteilt, bis sich eine Stoßfront bildet, an der Dichte und Schalldruck unstetig vom 
Maximum zum Minimum springen [44]. Die Ausbildung dieser Unstetigkeit geht einher mit der Erzeugung hoher Frequenzen, die im ursprünglichen Spektrum der Schallwelle nicht enthalten sind. Einen solchen nichtlinearen Effekt kann die lineare photoakustische Wellengleichung nicht abbilden.

Die mit der Frequenz zunehmende Dämpfung [76] wirkt der Erzeugung hoher Frequenzen entgegen [44]. Aus einer Schallwelle entwickelt sich deshalb nur dann eine Stoßfront, wenn die Ausgangsamplitude der Schallwelle genügend hoch ist. Für geringe Dichteänderungen kann die Schallgeschwindigkeit näherungsweise als konstant $\left(c \simeq c_{0}\right)$ und die Schallausbreitung als linear angesehen werden. M. Frenz und G. Paltauf beschreiben in zwei Arbeiten die Erzeugung von Schallwellen in wässrigen Farbstofflösungen durch fasergeführte Pulse eines optischen parametrischen Oszillators $\left(t_{\mathrm{p}}=6 \mathrm{~ns}\right)$ [25, 67]. Mit den gewählten Bestrahlungen erzeugten sie Schallfeldamplituden bis zu 1 kbar. Zur Modellierung des Schallfeldes verwendeten die Autoren die photoakustische Wellengleichung und erzielten eine hohe Übereinstimmung zwischen gemessenen und gerechneten Daten. Die Gültigkeit des linearen Modellierungsansatzes ist demnach für Schallfeldamplituden bis zu 1 kbar empirisch gesichert. Die Schallgeschwindigkeit ist bei diesem Druck um 10\% gegenüber $c_{0}$ erhöht.

\subsection{Lösung der photoakustischen Wellenglei- chung}

Aus der Potentialtheorie ist das allgemeine, vollständige Lösungsintegral der photoakustischen Wellengleichung (3.2) bekannt [59]. Es hat die Form

$$
\Phi(\vec{q}, t)=-\frac{1}{4 \pi} \frac{\mu \Gamma}{\rho_{0} c_{0}^{2}} \int I\left(\vec{r}, t_{\mathrm{ret}}\right) \frac{1}{|\vec{q}-\vec{r}|} d^{3} r
$$

mit $I\left[\mathrm{~W} / \mathrm{mm}^{2}\right]$ für die einfallende Bestrahlungsstärke. Der Ortsvektor $\vec{q}$ beschreibt die Position des Betrachters, während $\vec{r}$ die Integrationsvariable über den Raum darstellt. Die Größe $t_{\text {ret }}$ ist um die Laufzeit „retardiert“, die eine Störung vom Quellenpunkt $\vec{r}$ zum Betrachter bei $\vec{q}$ benötigt, wenn die Ausbreitungsgeschwindigkeit $c_{0}$ beträgt:

$$
t_{\mathrm{ret}}=t-\frac{|\vec{q}-\vec{r}|}{c_{0}}
$$

Obwohl Gl. (3.9) die photoakustische Wellengleichung vollständig löst, ist sie wegen ihrer Komplexität zunächst von geringem praktischen Nutzen. Insbesondere ist es in- 
folge der Retardierung nur in Sonderfällen möglich, das Integral analytisch zu lösen [59]. Andererseits verlangt die numerische Durchführung der Integration einen sehr hohen Rechenaufwand, da sie über drei Dimensionen erfolgt. Eine mögliche Vereinfachung ergibt sich, falls die Raum- und Zeitabhängigkeit des Quellterms separabel sind, d.h. wenn zwei unabhängige Funktionen $f(\vec{x})$ und $g(t)$ zur Bestrahlungsstärke $I$ existieren, mit

$$
I(\vec{x}, t)=f(\vec{x}) g(t) .
$$

Im Fall der Laseranregung beschreibt $g(t)$ den Zeitverlauf des Laserpulses, während $f(\vec{x})$ axial durch das Absorptionsgesetz und lateral durch das Strahlprofil des Lasers festgelegt ist. Unter Voraussetzung von Gl. (3.11) läßt sich zunächst die Antwortfunktion $\Phi_{\delta}(\vec{x}, t)$ auf eine Impulsanregung $g(t)=\delta(t)$ bestimmen. Da die photoakustische Wellengleichung ein lineares System bezüglich des Quelltermes $I(\vec{x}, t)$ darstellt, beinhaltet die Impulsantwort $\Phi_{\delta}(\vec{x}, t)$ das vollständige Übertragungsverhalten des Systems im Frequenzraum. Für eine beliebige Anregung $g(t)$ kann damit die Lösung $\Phi(\vec{x}, t)$ aus der Faltungsoperation

$$
\Phi(\vec{x}, t)=\left[\Phi_{\delta}(\vec{x}, \tau) \otimes g(\tau)\right](t)
$$

gewonnen werden [72]. Im folgenden soll $\Phi_{\delta}(\vec{x}, t)$ durch Einsetzen des Anregungsimpulses $I(\vec{x}, t) \equiv f(\vec{x}) \delta(t)$ in das Lösungsintegral (3.9) ermittelt werden. Dazu betrachte man die Integration über den Raum in sphärischer Geometrie und wähle für die Integration ein inneres Koordinatensystem (kurz: KS), in dem der Ort $\vec{q}$ des Betrachters mit dem Koordinaten-Ursprung $\vec{o}$ zusammenfällt. Mit $r \equiv|\vec{r}|$ erhält Gl. (3.9) die Form

$$
\Phi_{\delta}(\vec{q}, t)=-\frac{1}{4 \pi} \frac{\mu \Gamma}{\rho_{0} c_{0}^{2}} \int f(\vec{r}) \frac{\delta\left(t-r / c_{0}\right)}{r} d^{3} r
$$

Während sich die Integrationsvariable $\vec{r}$ auf das innere KS bezieht, ist die Ortsvariable von $f(\vec{x})$ auf ein äußeres, von der Integration unabhängiges KS bezogen. Das innere KS ist gegenüber dem äußeren gerade um den Vektor $\vec{q}$ verschoben. Folglich kann die Funktion $f(\vec{x})$ durch eine einfache Koordinatentransformation auch im inneren KS dargestellt werden, was auf eine entsprechende Form $f(\vec{r})$ führt.

Die nächste Umformung erfolgt mit Hilfe der Regel $\delta(a x)=\delta(x) /|a|$ [29]. Hierzu wird $a=-1 / c_{0}$ gesetzt, was auf

$$
\delta\left(t-r / c_{0}\right)=c_{0} \delta\left(r-c_{0} t\right)
$$

führt. Gemäß den Eigenschaften der $\delta$-Funktion wird das Integral über $\vec{r}$ nur dort ausgewertet, wo das Argument der $\delta$-Funktion identisch Null ist, d.h. auf der Oberfläche einer 
Kugel mit Radius $r=c_{0} t$. Damit bleibt:

$$
\Phi_{\delta}(\vec{q}, t)=-\left.\frac{1}{4 \pi} \frac{\mu \Gamma}{\rho_{0} c_{0}^{2}} \frac{1}{t} \int f(\vec{r})\right|_{r=c_{0} t} \mathrm{~d}^{2} r
$$

Die Lösungsdarstellung mittels Impulsantwort hat den unmittelbaren Nutzen, daß sich der Integrationsbereich um eine Dimension verringert. Anders als bei der Ausgangslösung findet sich die Zeitabhängigkeit nicht mehr im Integranden, sondern bestimmt aufgrund der Bedingung $r=c_{0} t$ den zu integrierenden Bereich. Für den Druck als Zielgröße folgt mit Gl. (3.3), $p=-\rho_{0} \partial \Phi / \partial t$, die Impulsantwortfunktion

$$
p_{\delta}(\vec{q}, t)=\frac{1}{4 \pi} \frac{\mu \Gamma}{c_{0}^{2}} \frac{\partial}{\partial t}\left[\left.\frac{1}{t} \int f(\vec{r})\right|_{r=c_{0} t} \mathrm{~d}^{2} r\right]
$$

sowie die zugehörige Faltungsoperation

$$
p(\vec{q}, t) \equiv\left[p_{\delta}(\vec{q}, \tau) \otimes g(\tau)\right](t)=\int_{-\infty}^{\infty} p_{\delta}(\vec{q}, t-\tau) g(\tau) \mathrm{d} \tau
$$

zum Lösungsverlauf $p(\vec{q}, t)$ der thermoelastischen Drucktransiente am Ort $\vec{q}$ des Beobachters. Gl. (3.16) ist die eingangs erwähnte „Poisson'sche Formel“. Sie ist in der Akustik als allgemeine Lösung der homogenen Wellengleichung bei gegebenen Anfangswerten $p(\vec{x}, 0)$ und $\vec{v}(\vec{x}, 0)$ bekannt [46]. Da die Wasserprobe vor der Laseranregung in Ruhe ist, gilt für die Schallschnelle $\vec{v}(\vec{x}, 0)=0$, während $p(\vec{x}, 0)$ gerade durch das räumliche Anregungsprofil $f(\vec{x})$ gegeben ist [25]. Der Separationsansatz führt demnach die Lösung der inhomogenen Wellengleichung auf ein Anfangswerteproblem der homogenen Wellengleichung zurück.

Da die Laseranregung initial eine Erhöhung des Druckes bewirkt, ist der Quellterm $f(\vec{r})$ im Integral der Poisson'schen Formel eine positive Funktion, die außerhalb der optischen Zone gegen Null strebt. Die endliche Ausdehnung dieser initialen Druckerhöhung hat zur Folge, daß ihre weitere Ausbreitung der Beugung unterliegt. Die Poisson'sche Formel quantifiziert diesen Effekt wie folgt: Für einen Betrachter am Ort $\vec{q}$ liefert das Integral $\left.\int f(\vec{r})\right|_{r=c_{0} t} \mathrm{~d}^{2} r$ einen positiven Wert, solang die Integrationssphäre mit Mittelpunkt $\vec{q}$ und Radius $r=c_{0} t$ die optische Zone schneidet, in $\operatorname{der} f(\vec{r})>0$ gilt. Mit zunehmender Zeit wächst die Integrationssphäre jedoch über die optische Zone hinaus. Dort ist $f(\vec{r})=0$, so daß auch das Integral auf Null abfällt. Da sich die Impulsantwort aus der zeitlichen Ableitung dieses Verlaufs bildet, ergibt sich für sie selbst eine bipolare Form, beginnend 
mit einer Druckphase und gefolgt von einer Zugphase. Für das Integral gilt dabei

$$
\int_{0}^{\infty} p_{\delta}(\vec{q}, t) \mathrm{d} t \propto\left[\left.\frac{1}{t} \int f(\vec{r})\right|_{r=c_{0} t} \mathrm{~d}^{2} r\right]_{0}^{\infty}=0 .
$$

Der bipolare Verlauf bleibt nach der Faltung mit dem zeitlichen Laserpulsprofil erhalten.

\section{Schallreflexion an der Quarz-Wasser-Grenzfläche}

Die thermoelastische Schallwelle trifft bei ihrer Ausbreitung auf die Quarz-WasserGrenzfläche und wird dort teilreflektiert. Vorzeichen und Betrag des zugehörigen Reflexionskoeffizienten $R_{\mathrm{QW}}$ ergeben sich gemäß

$$
R_{\mathrm{QW}}=\frac{Z_{\mathrm{Q}}-Z_{\mathrm{W}}}{Z_{\mathrm{Q}}+Z_{\mathrm{W}}}
$$

aus den akustischen Impedanzen $Z_{\mathrm{Q}, \mathrm{w}}=\left.\rho_{0} c_{0}\right|_{\mathrm{Q}, \mathrm{w}}$ der beteiligten Medien Quarz und Wasser [38]. Mit den in Tabelle 3.1 genannten Werten berechnet sich der akustische Reflexionskoeffizient $\mathrm{zu}$

$$
R_{\mathrm{QW}}=+0.796 \text {. }
$$

Aus dem positiven Vorzeichen geht hervor, daß die Reflexion an der Grenzfläche ohne Phasensprung erfolgt. Thermoelastische Zuganteile, die bei Strahlungsführung in eine flüssige Umgebung entstehen, sind daher ausschließlich auf die akustischen Beugungseffekte zurückzuführen, wie sie im Zusammenhang mit der Poisson'schen Formel (3.16) diskutiert wurden.

\begin{tabular}{|l|l|l|c|}
\hline & $\rho_{0}\left[\mathrm{~kg} / \mathrm{m}^{3}\right]$ & $c_{0}[\mathrm{~m} / \mathrm{s}]$ & $Z\left[\mathrm{~kg} /\left(\mathrm{m}^{2} \mathrm{~s}\right)\right]$ \\
\hline \hline syn. Quarz & 2202 & 5940 & $1.31 \cdot 10^{7}$ \\
\hline Wasser & 998 & 1480 & $1.48 \cdot 10^{6}$ \\
\hline
\end{tabular}

Tabelle 3.1: Akustische Impedanz $Z=\rho_{0} c_{0}$ von synthetischem Quarz und Wasser (Materialdaten aus $[34,75])$.

Zur Erzeugung des reflektierten Wellenanteils im Modell wird der Quellterm um einen fiktiven Anteil erweitert, der durch Spiegelung an der Grenzfläche entsteht [6, 66]. Für den separierten Raumanteil $f(\vec{x})$ der Bestrahlungsstärke $I(\vec{x}, t)$ führt dies auf die Darstellung

$$
f= \begin{cases}f(x, y, z) & : \quad z \geq 0 \\ R_{\mathrm{QW}} f(x, y,-z) & : \quad z<0\end{cases}
$$


Hierbei liegt die Grenzfläche bei $z=0$ und die Wasserprobe bei $z>0$, so daß der Ausdruck zu $z<0$ dem gespiegelten Anteil entspricht, in dem die reflexionsbedingten Änderungen der Amplitude durch den Vorfaktor $R_{\mathrm{QW}}$ berücksichtigt sind. Die $z$-Abhängigkeit von $f(\vec{x})$ ist durch das Absorptionsgesetz beschrieben, während die $(x, y)$-Abhängigkeit das Strahlprofil $H(x, y)$ des Lasers abbildet. Für einen parallelen Strahl erhält die Funktion $f(\vec{x})$ so die Darstellung

$$
f(x, y, z)=H(x, y) \cdot \begin{cases}\exp (-\mu z) & : \quad z \geq 0 \\ R_{\mathrm{QW}} \exp (+\mu z) & : \quad z<0\end{cases}
$$

Diese Darstellung kann in guter Näherung auch für divergente Strahlen eingesetzt werden, so lange die Divergenz gering über die Eindringtiefe der betrachteten Laserwellenlänge ausfällt.

\section{Übergang zu Kugelkoordinaten}

Aufgrund des sphärischen Integrationsgebietes in der Poisson'schen Formel ist eine Integration in Kugelkoordinaten zweckmäßig. Mit dem zugehörigen Oberflächenelement [29]

$$
\mathrm{d}^{2} r=r^{2} \sin (\vartheta) \mathrm{d} \varphi \mathrm{d} \vartheta
$$

ergibt sich aus Gl. (3.16) die Darstellung

$$
p_{\delta}(\vec{q}, t)=\frac{\mu \Gamma}{4 \pi} \frac{\partial}{\partial t}\left[\left.t \int_{0}^{\pi} \sin (\vartheta) \int_{0}^{2 \pi} f(r, \vartheta, \varphi)\right|_{r=c_{0} t} \mathrm{~d} \varphi \mathrm{d} \vartheta\right]
$$

Wie zu Gl. (3.14) erläutert wurde, sind die Kugelkoordinaten $(r, \vartheta, \varphi)$ einem inneren KS zugeordnet. Das innere KS wird jeweils so gewählt, daß der Koordinaten-Ursprung und der Ort $\vec{q}=\left(q_{x}, q_{y}, q_{z}\right)$ des Betrachters zusammenfallen. Das äußere KS zum Ortsvektor $\vec{x}=(x, y, z)$ ist fest und liegt wie in Abbildung 3.1 gezeigt. Es dient zur integrationsunabhängigen Darstellung der Anregung $f(\vec{x})$ gemäß Gl. (3.22). Auch der Ort des Beobachters $\vec{q}$ bezieht sich auf das äußere KS. Der Übergang ins innere KS erfolgt über die Beziehung

$$
\left.f(r, \vartheta, \varphi)\right|_{\vec{q}} \equiv f\left(x\left(r, \vartheta, \varphi, q_{x}\right), y\left(r, \vartheta, \varphi, q_{y}\right), z\left(r, \vartheta, q_{z}\right)\right)
$$




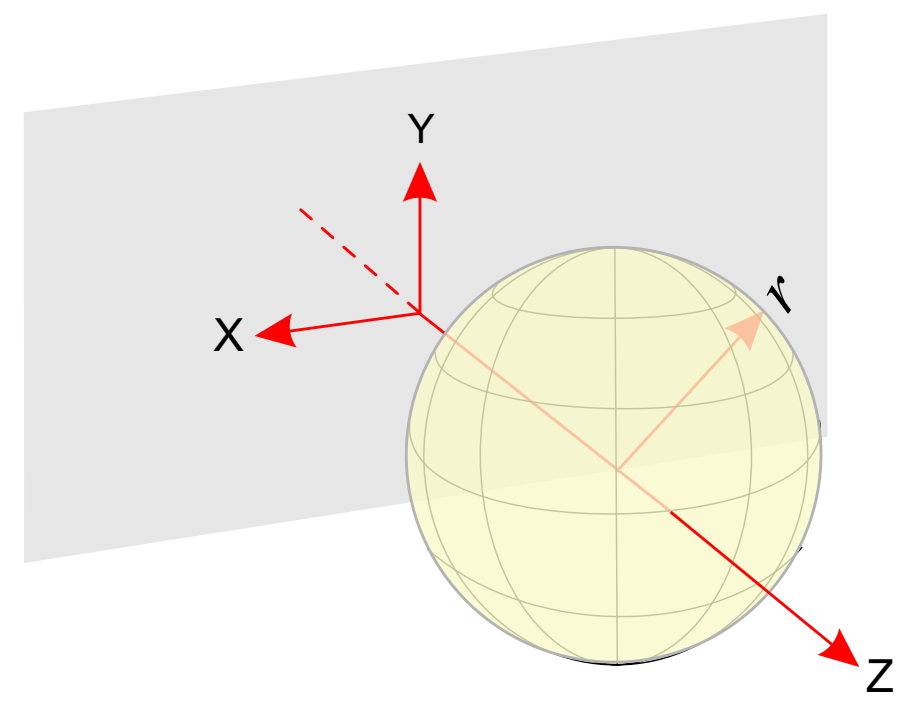

Abb. 3.1: Darstellung des äußeren KS zum Ortsvektor $\vec{x}=(x, y, z)$ sowie einer Integrationssphäre mit Radius $r=c_{0} t$. Die Ebene $z=0$ befindet sich an der Quarz-Wasser-Grenzfläche (grau unterlegt), die $z$-Achse entspricht der Strahlachse und weist in Richtung Wasser.

mit den Transformationsgleichungen [29]

$$
\begin{aligned}
x\left(r, \vartheta, \varphi, q_{x}\right) & =r \sin (\vartheta) \cos (\varphi)+q_{x}, \\
y\left(r, \vartheta, \varphi, q_{y}\right) & =r \sin (\vartheta) \sin (\varphi)+q_{y} \text { und } \\
z\left(r, \vartheta, q_{z}\right) & =r \cos (\vartheta)+q_{z} .
\end{aligned}
$$

Für die Winkel gilt $\varphi \in[0,2 \pi]$ und $\vartheta \in[0, \pi]$. Nach Anwendung der Koordinatentransformation auf Gl. (3.22) ergibt sich der Integrand zu

$$
\begin{aligned}
\left.f(r, \vartheta, \varphi)\right|_{\vec{q}}= & \left.H(r, \vartheta, \varphi)\right|_{q_{x}, q_{y}} \cdot \\
& \left\{\begin{array}{lll}
\exp \left(-\mu\left(r \cos (\vartheta)+q_{z}\right)\right) & : r \cos (\vartheta)+q_{z} \geq 0 \\
R_{\mathrm{QW}} \exp \left(+\mu\left(r \cos (\vartheta)+q_{z}\right)\right) & : & r \cos (\vartheta)+q_{z}<0
\end{array}\right.
\end{aligned}
$$

Aus dieser Darstellung geht hervor, daß der Übergang vom initialen Quellterm (obere Formelhälfte) zum gespiegelten Anteil (untere Formelhälfte) nun eine Funktion des Radius $r=c_{0} t$, des Winkels $\vartheta$ und des senkrechten Abstandes $q_{z}$ zur Grenzfläche ist. Für gegebene Größen $r$ und $q_{z}$ gibt es deshalb einen Grenzwinkel $\vartheta_{\mathrm{G}}$, der sich aus der Definitionsgleichung

$$
r \cos \left(\vartheta_{\mathrm{G}}\right)+q_{z} \equiv 0
$$


bestimmt und den Wechsel zwischen beiden Quelltermanteilen markiert. Voraussetzung für die Existenz einer Lösung von Gl. (3.27) ist die Bedingung $r>q_{z}$, denn anderenfalls erfolgt die Integration noch vollständig im initialen Quelltermanteil, wie in Abbildung 3.2 dargestellt ist. Insgesamt führt dies auf einen Grenzwinkel $\vartheta_{\mathrm{G}} \in[\pi / 2, \pi]$ von der Form

$$
\vartheta_{\mathrm{G}}=\left\{\begin{array}{ll}
\arccos \left(-q_{z} / r\right) & : \quad r>q_{z} \\
\pi & : \quad r \leq q_{z}
\end{array},\right.
$$

der für $r>q_{z}$ die Definitionsgleichung (3.27) löst und für $r \leq q_{z}$ der oberen Integrationsgrenze zum Winkel $\vartheta$ entspricht.
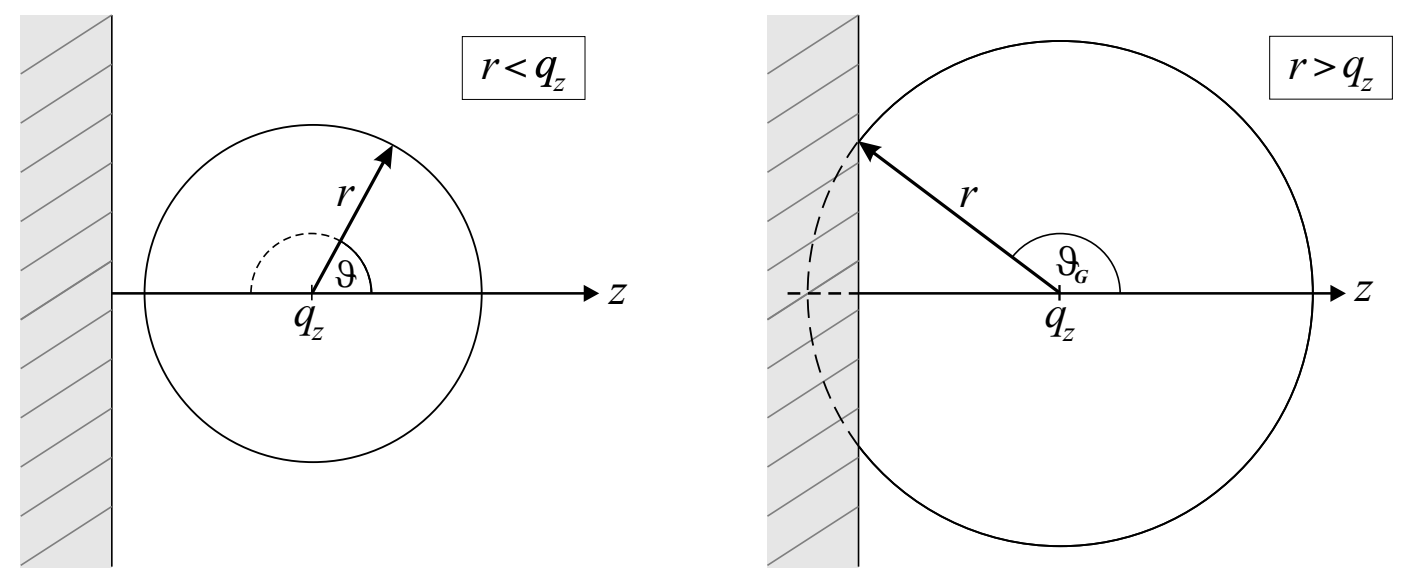

Abb. 3.2: Seitenansicht der Integrationssphäre mit Mittelpunkt $\vec{q}=\left(q_{x}, q_{y}, q_{z}\right)$. Der grau schraffierte Bereich markiert den eingetauchten Quarzkörper, die Grenzfläche zum Wasser befindet sich bei $z=0$. Links: Integration innerhalb des initialen Quellterms. Rechts: Übergang vom initialen zum gespiegelten Quelltermanteil am Grenzwinkel $\vartheta_{\mathrm{G}}$.

\section{Temperaturabhängigkeit von Absorption und Grüneisenkoeffizient}

Bei der Herleitung der Poisson'schen Formel aus der photoakustischen Wellengleichung wurde vorausgesetzt, daß es sich bei der Absorption und dem Grüneisenkoeffizienten um Konstanten handelt. Tatsächlich hat die Temperaturabhängigkeit beider Größen verschiedenen Einfluß auf das Druckmodell:

Wie in Unterabschnitt 2.2.1 erläutert, führt die Temperaturabhängigkeit der Absorption noch während der Laseranregung zu einer Änderung des Absorptionsprofils („Ausbleichen" der Wasserprobe). Räumliche und zeitliche Entwicklung der absorbierten Bestrahlung sind hierdurch nicht mehr voneinander getrennt, so daß der Separationsansatz 
$I(\vec{x}, t)=f(\vec{x}) g(t)$ streng genommen nicht gilt. Eine exakte Modellierung der Schallfeldentwicklung kann folglich nur auf Grundlage des vollständigen, 3-dimensionalen Lösungsintegrals (3.9) geschehen. Dies führt auf eine komplexe numerische Lösungsbehandlung, beginnend bei der Darstellung des zeitabhängigen Absorptionsprofils mittels iterativer Berechnungsverfahren [47].

Bevor die Schallfeldentwicklung mit letzter Genauigkeit, aber erheblichem numerischen Aufwand berechnet wird, sei das Absorptionsprofil im folgenden als näherungsweise konstant von der Form $\propto \exp (-\mu z)$ angenommen, so daß der Separationsansatz und damit die Poisson'sche Formel weiter zur Lösungsdarstellung verwendet werden können. Dieses Vorgehen, die Schallentwicklung so weit wie möglich analytisch darzustellen, erlaubt ein besseres Verständnis vom Lösungsverhalten der photoakustischen Wellengleichung. $\mathrm{Ob}$ die Näherungslösungen die Realität dabei hinreichend gut abbilden, wird im Vergleich des Modells mit dem Experiment beantwortet (Kapitel 5).

Um die Änderungen mit der Temperatur in einem gewissen Maße zu berücksichtigen, wird für den Absorptionskoeffizienten ein über die Temperatur gemittelter Wert

$$
\bar{\mu}=\frac{\int_{T_{0}}^{T_{\mathrm{E}}} \mu(T) \mathrm{d} T}{T_{\mathrm{E}}-T_{0}}
$$

eingesetzt. Hierbei ist $T_{0}$ die Raumtemperatur und $T_{\mathrm{E}}$ die zur gegebenen Bestrahlung $H$ zugehörige Endtemperatur. Für den Linearverlauf $\mu(T)=a+b T$ (Unterabschnitt 2.2.1) gilt dabei

$$
\bar{\mu}=\frac{\mu\left(T_{\mathrm{E}}\right)+\mu\left(T_{0}\right)}{2} .
$$

Die Temperaturabhängigkeit des Grüneisenkoeffizienten $\Gamma$ fließt über den Quellterm in die photoakustische Wellengleichung (3.2) ein. Dies geschieht, indem sich die Zeitableitung im Quellterm auf $\Gamma$ ausdehnt [30]. Die photoakustische Wellengleichung (3.2) lautet damit

$$
\nabla^{2} \Phi-\frac{1}{c_{0}^{2}} \frac{\partial^{2} \Phi}{\partial t^{2}}=\frac{\bar{\mu}}{\rho_{0} c_{0}^{2}} \frac{\partial}{\partial t}\left[\left.\Gamma(H)\right|_{\mu} H(\vec{x}, t)\right],
$$

wenn der Grüneisenkoeffizient als explizite Funktion der Bestrahlung dargestellt wird: $\left.\Gamma(H)\right|_{\mu} \equiv \Gamma\left(\left.T(H)\right|_{\mu}\right)$. Die innere Funktion $\left.T(H)\right|_{\mu}$ beschreibt dabei die Temperaturentwicklung zur Absorption $\mu$ der jeweiligen Laserwellenlänge. Für eine allgemein als Potenzreihe definierte Abhängigkeit

$$
\Gamma(H)=\Gamma_{0}+\Gamma_{1} H+\Gamma_{2} H^{2}+\ldots
$$

folgt die Darstellung 


$$
\nabla^{2} \Phi-\frac{1}{c_{0}^{2}} \frac{\partial^{2} \Phi}{\partial t^{2}}=\frac{\bar{\mu}}{\rho_{0} c_{0}^{2}}\left(\Gamma_{0} \frac{\partial H}{\partial t}+\Gamma_{1} \frac{\partial\left(H^{2}\right)}{\partial t}+\Gamma_{2} \frac{\partial\left(H^{3}\right)}{\partial t}+\ldots\right)
$$

Das erste Glied auf der rechten Seite entspricht der Ausgangssituation zu einem konstanten Grüneisenkoffizienten $\Gamma=\Gamma_{0}$ und einem Quellterm direkt proportional zur Bestrahlungsstärke $I=\partial H / \partial t$. Durch die höheren Terme ist diese Proportionalität aufgehoben, weshalb die Temperaturabhängigkeit des Grüneisenkoeffizienten auch als thermische Nichtlinearität bezeichnet wird [52]. Im Unterschied zu den akustischen Nichtlinearitäten verletzt die thermische zwar nicht die Linearität der Schallausbreitung, wirkt sich aber auf Amplitude und Verlauf der Schallentwicklung aus.

Durch Einsetzen eines Quellterms gemäß Gl. (3.33) in das vollständige Lösungsintegral (3.9) der photoakustischen Wellengleichung folgt für jedes Reihenglied ein eigenständiges Lösungsintegral, das mittels Poisson'scher Formel gelöst werden kann. Die Gesamtlösung ergibt sich durch Superposition aller gefundenen Teillösungen.

Ist die Bestrahlungsstärke $I(\vec{x}, t)=f(\vec{x}) g(t)$ separabel und damit auch die Bestrahlung $H(\vec{x}, t)=f(\vec{x}) \int_{0}^{t} g(\tau) \mathrm{d} \tau$, so sind es alle Glieder in der Reihenentwicklung (3.33) des Quellterms, denn aus den Ableitungen

$$
\frac{\partial\left(H^{n}\right)}{\partial t}=n H^{n-1} \frac{\partial H}{\partial t}=n(H(\vec{x}, t))^{n-1} I(\vec{x}, t)
$$

lassen sich für alle $n=1,2, \ldots$ eine Ortsfunktion

$$
f_{n}(\vec{x}) \equiv n f^{n}(\vec{x})
$$

und eine Zeitfunktion

$$
g_{n}(t) \equiv g(t)\left(\int_{-\infty}^{t} g(\tau) \mathrm{d} \tau\right)^{n-1}
$$

separieren. Jede Ortsfunktion $f_{n}(\vec{x})$ generiert dabei über die Poisson'sche Formel (3.16) eine eigene Impulsantwort $p_{\delta, n}(\vec{q}, t)$, die durch Faltung mit der zugehörigen Zeitfunktion $g_{n}(t)$ zum Lösungsverlauf $p_{n}(\vec{q}, t)$ des $n$-ten Reihengliedes führt.

Bei einer Reihenentwicklung bis zur Ordnung $n_{\max }$ ergibt sich die Gesamtlösung $p(\vec{q}, t)$ durch einfache Summation

$$
p(\vec{q}, t)=\sum_{n=1}^{n_{\max }} p_{n}(\vec{q}, t)
$$

über alle Einzellösungen. 


\subsubsection{Thermische Nichtlinearitäten bei der Tm- und Ho- Laseranregung}

Für die Anregung mit dem Tm- und Ho-Laser ist in Unterabschnitt 2.2.1 die Temperaturfunktion (2.10),

$$
\left.T(H)\right|_{\mu}=\frac{1}{b}\left[\mu\left(T_{0}\right) \exp \left(\frac{b H}{\rho C}\right)-a\right],
$$

zu den Absorptionsverläufen $\mu(T)=a+b T$ der jeweiligen Laserwellenlänge entwickelt worden. Aus dieser laserspezifischen Temperaturentwicklung $\left.T(H)\right|_{\mu}$ resultieren über die Verkettung $\left.\Gamma(H)\right|_{\mu}=\left.\Gamma(T) \circ T(H)\right|_{\mu}$ laserspezifische Koeffizienten $\Gamma_{1}, \Gamma_{2}, \Gamma_{3}$ in der Reihenentwicklung des photoakustischen Quellterms. Der Koeffizient $\Gamma_{0}$ ist hingegen konstant gleich dem Wert in der ungestörten Wasserprobe. Die Temperaturabhängigkeit $\Gamma(T)$ des Grüneisenkoeffizienten ist als Funktion zwischen $20^{\circ} \mathrm{C}$ und $300^{\circ} \mathrm{C}$ bei G. Paltauf et. al. [65] beschrieben (Abbildung 3.3). Durch Einsetzen von $\Gamma(T)$ in Gl. (2.10) und nu-

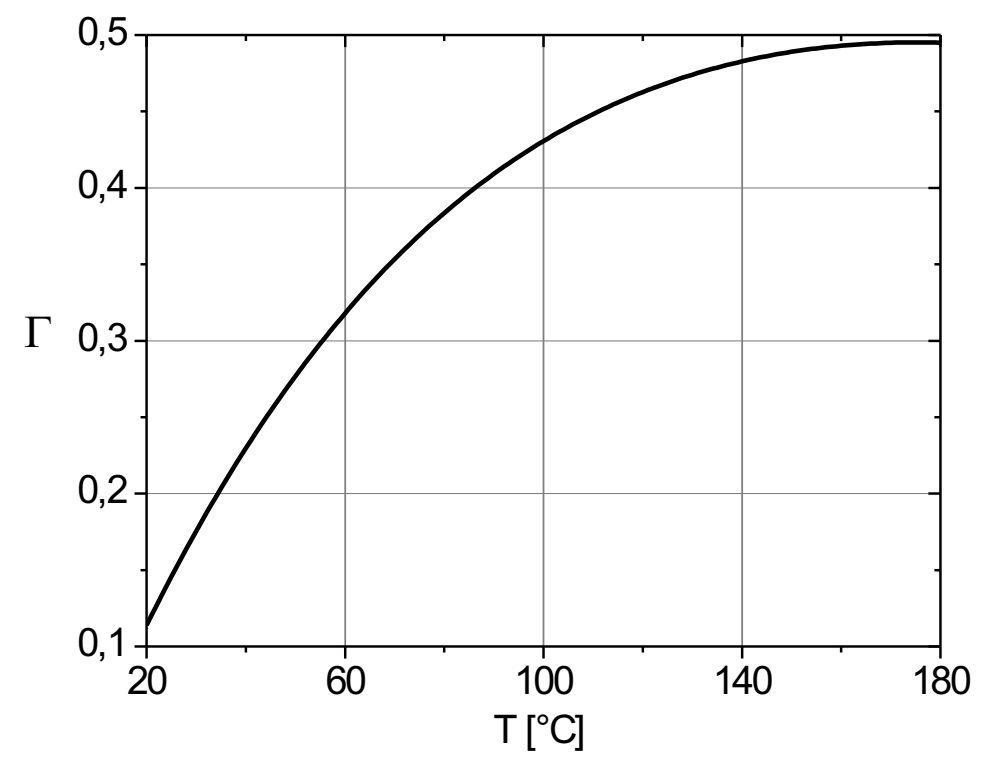

Abb. 3.3: Temperaturabhängigkeit des Grüneisenkoeffizienten von Wasser [65], mit $\Gamma\left(20^{\circ} \mathrm{C}\right)=$ 0.1138 .

merische Anpassung eines Regressionspolynoms an die Resultierende $\left.\Gamma(H)\right|_{\mu}$ folgen zu beiden Laserwellenlängen die in Tabelle 3.2 aufgelisteten Reihenkoeffizienten. Die Ausdehnung des Regressionspolynoms bis zur 7. Ordnung erwies sich als erforderlich, um Abweichungen zur Ausgangsfunktion von $(\Delta \Gamma / \Gamma)<0.5 \%$ sicherzustellen. Die Gültigkeit des Regressionspolynoms ist beschränkt auf Bestrahlungen bis $\simeq 170 \mathrm{~mJ} / \mathrm{mm}^{2}(\mathrm{Tm})$ bzw. $300 \mathrm{~mJ} / \mathrm{mm}^{2}(\mathrm{Ho})$, entsprechend der Gültigkeit der Funktionen $\left.T(H)\right|_{\mu}$. 


\begin{tabular}{|l|r|r|r|r|c|}
\hline & \multicolumn{2}{|c|}{ Thulium } & \multicolumn{2}{c|}{ Holmium } & \\
\hline Koeffizient & Wert & Fehler & Wert & Fehler & Einheit \\
\hline \hline$\Gamma_{0}$ & $1.138 \cdot 10^{-01}$ & $4 \cdot 10^{-05}$ & $1.138 \cdot 10^{-1}$ & $3 \cdot 10^{-07}$ & \\
\hline$\Gamma_{1}$ & $1.016 \cdot 10^{-02}$ & $5 \cdot 10^{-06}$ & $5.04 \cdot 10^{-3}$ & $5 \cdot 10^{-08}$ & $\mathrm{~mm}^{2} / \mathrm{mJ}$ \\
\hline$\Gamma_{2}$ & $-1.269 \cdot 10^{-04}$ & $2 \cdot 10^{-07}$ & $-3.0563 \cdot 10^{-5}$ & $2 \cdot 10^{-09}$ & $\mathrm{~mm}^{4} / \mathrm{mJ}^{2}$ \\
\hline$\Gamma_{3}$ & $9.96 \cdot 10^{-07}$ & $4 \cdot 10^{-09}$ & $1.2008 \cdot 10^{-7}$ & $4 \cdot 10^{-11}$ & $\mathrm{~mm}^{6} / \mathrm{mJ}^{4}$ \\
\hline$\Gamma_{4}$ & $-5.19 \cdot 10^{-09}$ & $4 \cdot 10^{-11}$ & $-3.428 \cdot 10^{-10}$ & $4 \cdot 10^{-13}$ & $\mathrm{~mm}^{8} / \mathrm{mJ}^{6}$ \\
\hline$\Gamma_{5}$ & $1.72 \cdot 10^{-11}$ & $2 \cdot 10^{-13}$ & $6.98 \cdot 10^{-13}$ & $2 \cdot 10^{-15}$ & $\mathrm{~mm}^{10} / \mathrm{mJ}^{6}$ \\
\hline$\Gamma_{6}$ & $-3.21 \cdot 10^{-14}$ & $5 \cdot 10^{-16}$ & $-9.13 \cdot 10^{-16}$ & $5 \cdot 10^{-18}$ & $\mathrm{~mm}^{12} / \mathrm{mJ}^{8}$ \\
\hline$\Gamma_{7}$ & $2.58 \cdot 10^{-17}$ & $5 \cdot 10^{-19}$ & $5.7 \cdot 10^{-19}$ & $1 \cdot 10^{-20}$ & $\mathrm{~mm}^{14} / \mathrm{mJ}^{10}$ \\
\hline
\end{tabular}

Tabelle 3.2: Reihenkoeffizienten zu $\left.\Gamma(H)\right|_{\mu}=\Gamma_{0}+\Gamma_{1} H+\Gamma_{2} H^{2}+\ldots+\Gamma_{7} H^{7}$, mit $\Gamma_{0}=\Gamma\left(20^{\circ} \mathrm{C}\right)$.

\section{Zeitfunktionen zum gütegeschalteten Tm- und Ho-Laserpuls}

Wie in Abschnitt 2.1 dargestellt, folgen die zeitlichen Profile der gütegeschalteten Tmund Ho-Laserpulse in guter Näherung einer Gaußkurve

$$
g(t)=g_{0} \exp \left(-\ln 2\left(\frac{2 t}{t_{\mathrm{p}}}\right)^{2}\right),
$$

zur jeweiligen Pulsdauer $t_{\mathrm{p}}(\mathrm{FWHM})$. Im weiteren soll $g(t)$ die Normierungsbedingung

$$
\int_{-\infty}^{\infty} g(\tau) \mathrm{d} \tau=1
$$

erfüllen, was durch Wahl von

$$
g_{0}=\frac{2}{t_{\mathrm{p}}} \sqrt{\frac{\ln 2}{\pi}}
$$

erreicht wird. Die Normierung stellt sicher, daß die Gesamtpulsenergie $E$ nicht zweifach in die Berechnungen des Schallfeldes eingeht, da sie nachfolgend in der Darstellung der Strahlprofile $H(x, y)$ bereits berücksichtigt ist. Die Integration von $g(t)$ führt auf eine Gaußsche Fehlerfunktion erf $(x)$ von der Form

$$
\int_{-\infty}^{t} g(\tau) \mathrm{d} \tau=\frac{1}{2}\left(1+\operatorname{erf}\left(\frac{2 t}{t_{\mathrm{p}}} \sqrt{\ln (2)}\right)\right) .
$$

Zuammen mit Gl. (3.38) lassen sich hieraus die Zeitfunktionen $g_{n}(t)$ (3.36) zu allen Quelltermgliedern entwickeln. 


\subsection{Berechnung zum Gaußschen Laserstrahl}

Das Strahlprofil des radialsymmetrischen Gaußschen Laserstrahls sei durch

$$
H(x, y)=H_{0} \exp \left(-2 \frac{x^{2}+y^{2}}{w^{2}}\right), \quad \text { mit } \quad H_{0}=\frac{2 E}{\pi w^{2}}
$$

dargestellt. Darin ist $E$ die Energie des Laserpulses und $w$ der Gaußsche Strahlradius zum $1 / \mathrm{e}^{2}$-Abfall. Mit dem gewählten $H_{0}$ ist

$$
\int_{-\infty}^{\infty} \int_{-\infty}^{\infty} H(x, y) \mathrm{d} x \mathrm{~d} y=E
$$

so daß die applizierte Pulsenergie nicht mehr im Zeitanteil $g(t)$ der Bestrahlung berücksichtigt werden muß (Gl. (3.40)). Im folgenden werden die Lösungen $p_{\delta, n}(\vec{q}, t)$ der Poisson'schen Formel zu allen Reihengliedern $\Gamma_{n-1}\left(\partial\left(H^{n}\right) / \partial t\right)$ des photoakustischen Quellterms gesucht. Stellvertretend für die Gesamtentwicklung des Schallfeldes werden die Lösungen auf der Strahlachse und in der Eintrittsebene $z=0$ des Laserstrahls betrachtet. Die Eintrittsebene ist gleichbedeutend mit der Grenzfläche zwischen strahlungsführendem Quarzkörper und der Wasserprobe (Abbildung 3.2). Die Lösungen der Poisson'schen Formel für Punkte auf der Strahlachse erlauben einen direkten Vergleich mit experimentell bestimmten Drucktransienten im akustischen Fernfeld (Unterabschnitt 5.1.2), während die Untersuchungen zur Schallentwicklung in der Eintrittsebene die Diskussion zum thermodynamischen Zustandsverlauf der bestrahlten Probe unterstützen (Kapitel 4).

\subsubsection{Schallentwicklung auf der Strahlachse}

Bei der Berechnung der Poisson'schen Formel (3.16) in einem Punkt $\vec{q}=\left(0,0, q_{z}\right)$ der Strahlachse kommt die Radialsymmetrie des Gaußschen Strahlprofils zum Tragen. Für $q_{x}=q_{y}=0$ ergibt sich nach Transformation in das innere KS der Ausdruck

$$
H(r, \vartheta)=H_{0} \exp \left(-2 \frac{r^{2} \sin ^{2}(\vartheta)}{w^{2}}\right)
$$

in dem der Winkel $\varphi$ nicht mehr auftritt. Die Integration von $f_{n}=f_{n}\left(r, \vartheta, q_{z}\right)$ über $\varphi$ liefert damit einen Faktor $2 \pi$ und die Poisson'sche Formel (3.24) vereinfacht sich zu

$$
p_{\delta, n}\left(q_{z}, t\right)=\frac{\bar{\mu} \Gamma_{n-1}}{2} \frac{\partial}{\partial t}\left[\left.t \int_{0}^{\pi} \sin (\vartheta) f_{n}\left(r, \vartheta, q_{z}\right)\right|_{r=c_{0} t} \mathrm{~d} \vartheta\right] \text {. }
$$


An die Stelle eines sphärischen Integrationsgebietes ist nun eine Integralkurve getreten, die einen Halbkreis mit Radius $r=c_{0} t$ beschreibt und durch $\vartheta$ parametrisiert ist. Wie in Abbildung 3.2 dargestellt, wechselt die Form des Integranden $f_{n}\left(r, \vartheta, q_{z}\right)$ am Grenzwinkel $\vartheta=\vartheta_{\mathrm{G}}$ zwischen dem initialen und dem gespiegelten Quelltermanteil; es ist

$$
f_{n}\left(r, \vartheta, q_{z}\right)=n H^{n}(r, \vartheta) \cdot \begin{cases}\exp \left(-\bar{\mu} n\left(r \cos (\vartheta)+q_{z}\right)\right) & : \vartheta \leq \vartheta_{\mathrm{g}} \\ \left(R_{\mathrm{QW}}\right)^{n} \exp \left(+\bar{\mu} n\left(r \cos (\vartheta)+q_{z}\right)\right) & : \vartheta>\vartheta_{\mathrm{g}}\end{cases}
$$

In der Poisson'schen Formel werden durch $\vartheta_{\mathrm{G}}$ zwei Teilintegrale definiert. Es sei

$$
\begin{aligned}
& J_{-}(r, n) \equiv t \int_{0}^{\vartheta_{\mathrm{G}}} \sin (\vartheta) \exp \left(-2 n \frac{r^{2} \sin ^{2}(\vartheta)}{w^{2}}-\bar{\mu} n r \cos (\vartheta)\right) \mathrm{d} \vartheta, \quad \text { und } \\
& J_{+}(r, n) \equiv t \int_{\vartheta_{\mathrm{G}}}^{\pi} \sin (\vartheta) \exp \left(-2 n \frac{r^{2} \sin ^{2}(\vartheta)}{w^{2}}+\bar{\mu} n r \cos (\vartheta)\right) \mathrm{d} \vartheta
\end{aligned}
$$

Die Poisson'sche Formel erhält damit die Gestalt

$$
p_{\delta, n}\left(q_{z}, t\right)=\frac{\bar{\mu} n \Gamma_{n-1}\left(H_{0}\right)^{n}}{2}\left[\mathrm{e}^{-\bar{\mu} n q_{z}} \frac{\partial J_{-}(r, n)}{\partial t}+\left(R_{\mathrm{QW}}\right)^{n} \mathrm{e}^{+\bar{\mu} n q_{z}} \frac{\partial J_{+}(r, n)}{\partial t}\right] .
$$

Das Integral $J_{+}(r, n)$ existiert hierbei erst für Radien $r>q_{z}$, bzw. Zeiten $t>q_{z} / c_{0}$. Anderenfalls ist $J_{+}(r, n)=0$, denn nach Gl. (3.28) nimmt der Grenzwinkel in diesem Fall den Wert $\vartheta_{\mathrm{G}}=\pi$ an.

Die Lösung der beiden Integrale $J_{ \pm}(r, n)$ stellt das Kernproblem der Berechnungen auf der Strahlachse dar: Werden die Integrale numerisch gelöst, so muß auch die nachfolgende Zeitableitung in der Poisson'schen Formel numerisch durchgeführt werden, was insgesamt einen erheblichen Rechenaufwand bedeutet. Findet sich dagegen ein analytischer Lösungsweg für die Integrale, dann ist auch die Zeitableitung analytisch durchführbar und es läßt sich explizit eine Lösungsformel für die Schallentwicklung auf der Strahlachse angeben. Da es in der Literatur keinen Hinweis auf einen analytischen Lösungsweg gibt, wurden die Integrale $J_{ \pm}(r, n)$ im Rahmen dieser Arbeit eingehend auf eine solche Lösungsmöglichkeit untersucht. Nach einer Reihe geeigneter Variablensubstitionen konnten die Integrale hierbei auf die sog. ,,imaginäre Fehlerfunktion“

$$
\operatorname{erfi}(\zeta(r))=\frac{2}{\sqrt{\pi}} \int_{0}^{\zeta(r)} \mathrm{e}^{\tau^{2}} \mathrm{~d} \tau
$$


zurückgeführt werden, worin $\zeta(r)$ für eine einfache, lineare Funktion des Radius steht. Die imaginäre Fehlerfunktion leitet sich gemäß erfi $(x)=\operatorname{erf}(i x) / i$ aus der reellen Fehlerfunktion $\operatorname{erf}(x)$ ab und ist ebenfalls reellwertig. Die zeitliche Ableitung von Gl. (3.50) läßt sich mit Hilfe der Ableitungsregeln für Parameterintegrale [29] bestimmen und führt auf einen Ausdruck $\propto \exp \left(\zeta^{2}(r)\right)$.

Die analytische Rückführung der Integrale $J_{ \pm}(r, n)$ auf die imaginäre Fehlerfunktion ist in Anhang A.1 dargestellt. Die anschließend durchgeführte Zeitableitung wurde mit Hilfe der symbolischen Berechnungsmöglichkeiten der Mathematiksoftware „Mathematica“ (Wolfram Research, Version 4.0) auf Vollständigkeit und Richtigkeit überprüft. Bei der Darstellung der Lösungsformel sind wieder die beiden Fälle $r \leq q_{z}$ und $r>q_{z}$ für den Radius der Integrationskurve zu unterscheiden, entsprechend den Bedingungen $t \leq q_{z} / c_{0}$ und $t>q_{z} / c_{0}$. Im ersten Fall lautet die Lösungsformel

$$
\begin{aligned}
\left.p_{\delta, n}\left(q_{z}, t\right)\right|_{t \leq q_{z} / c_{0}}= & \frac{\bar{\mu} n \Gamma_{n-1}\left(H_{0}\right)^{n}}{2}\left[\mathrm{e}^{-\bar{\mu} n q_{z}} N_{n}(r)\left\{\operatorname{erfi}\left(a_{n}(r)\right)-\operatorname{erfi}\left(b_{n}(r)\right)\right\}+\right. \\
& \left.\mathrm{e}^{-\bar{\mu} n\left(q_{z}+r\right)}+\mathrm{e}^{-\bar{\mu} n\left(q_{z}-r\right)}\right]
\end{aligned}
$$

im zweiten Fall ist die Lösung von der Form

$$
\begin{aligned}
\left.p_{\delta, n}\left(q_{z}, t\right)\right|_{t>q_{z} / c_{0}}= & \frac{\bar{\mu} n \Gamma_{n-1}\left(H_{0}\right)^{n}}{2}\left[N_{n}(r)\left\{L_{n}(r)-K_{n}\left(q_{z}\right)\right\}+\right. \\
& \left.\mathrm{e}^{-\bar{\mu} n\left(q_{z}+r\right)}+\left(R_{\mathrm{QW}}\right)^{n} \mathrm{e}^{-\bar{\mu} n\left(r-q_{z}\right)}\right] .
\end{aligned}
$$

Die Funktionen $L_{n}(r), N_{n}(r), a_{n}(r)$ und $b_{n}(r)$ sowie die Größe $K_{n}\left(q_{z}\right)$ sind dabei wie folgt definiert:

$$
\begin{aligned}
L_{n}(r) & =\operatorname{erfi}\left(a_{n}(r)\right)\left[\mathrm{e}^{-\bar{\mu} n q_{z}}+\left(R_{\mathrm{QW}}\right)^{n} \mathrm{e}^{+\bar{\mu} n q_{z}}\right], \\
N_{n}(r) & =\frac{\sqrt{2 \pi n} r}{w} \exp \left(-n\left(\frac{2 r^{2}}{w^{2}}+\frac{\bar{\mu}^{2} w^{2}}{8}\right)\right), \\
b_{n}(r) & =\sqrt{n}\left(\frac{\sqrt{2} r}{w}+\frac{\bar{\mu} w}{\sqrt{8}}\right), \\
a_{n}(r) & =\sqrt{n}\left(\frac{\bar{\mu} w}{\sqrt{8}}-\frac{\sqrt{2} r}{w}\right) \text { und } \\
K_{n}\left(q_{z}\right) & =\mathrm{e}^{-\bar{\mu} n q_{z}} \operatorname{erfi}\left(b_{n}\left(q_{z}\right)\right)+\left(R_{\mathrm{QW}}\right)^{n} \mathrm{e}^{+\bar{\mu} n q_{z}} \operatorname{erfi}\left(a_{n}\left(q_{z}\right)\right) .
\end{aligned}
$$


Einen wichtigen Sonderfall bildet die Schallentwicklung im Ursprung $\vec{o}=(0,0,0)$. An diesem Punkt ist die Bestrahlung maximal, so daß hier auch die höchsten Schallfeldamplituden zu erwarten sind. Um diese zu bestimmen, wird $q_{z}$ in Gl. (3.52) Null gesetzt, woraus sich der folgende Ausdruck ergibt:

$$
\begin{aligned}
p_{\delta, n}(\vec{o}, t)= & \frac{\bar{\mu} n \Gamma_{n-1}\left(H_{0}\right)^{n}}{2}\left(1+\left(R_{\mathrm{QW}}\right)^{n}\right) \cdot \\
& {\left[N_{n}(r)\left\{\operatorname{erfi}\left(a_{n}(r)\right)-\operatorname{erfi}\left(\bar{\mu} w \sqrt{\frac{n}{8}}\right)\right\}+\mathrm{e}^{-\bar{\mu} n r}\right] . }
\end{aligned}
$$

\section{Übergang zur ebenen Welle}

Im Grenzfall einer lateral unendlich ausgedehnten Anregung $(w \rightarrow \infty)$ nimmt das Schallfeld die Form einer eindimensionalen, ebenen Welle an. Aufgrund der Grenzwerte

$$
\lim _{w \rightarrow \infty}\left[b_{n}(r)-a_{n}(r)\right]_{w}=\lim _{w \rightarrow \infty}\left[N_{n}(r)\right]_{w}=0
$$

und folglich

$$
\lim _{w \rightarrow \infty}\left[N_{n}(r)\left\{\operatorname{erfi}\left(a_{n}(r)\right)-\operatorname{erfi}\left(b_{n}(r)\right)\right\}\right]_{w}=\lim _{w \rightarrow \infty}\left[N_{n}(r)\left\{L_{n}(r)-K_{n}\left(q_{z}\right)\right\}\right]_{w}=0
$$

vereinfachen sich die Lösungsfunktionen (3.51), (3.52) zu

$$
\begin{aligned}
\left.p_{\delta, n}\left(q_{z}, t\right)\right|_{w \rightarrow \infty}= & \frac{\bar{\mu} n \Gamma_{n-1}\left(H_{0}\right)^{n}}{2} . \\
& \begin{cases}\mathrm{e}^{-\bar{\mu} n\left(q_{z}+r\right)}+\mathrm{e}^{-\bar{\mu} n\left(q_{z}-r\right)} & : t<q_{z} / c_{0} \\
\mathrm{e}^{-\bar{\mu} n\left(q_{z}+r\right)}+\left(R_{\mathrm{QW}}\right)^{n} \mathrm{e}^{-\bar{\mu} n\left(r-q_{z}\right)} & : t \geq q_{z} / c_{0}\end{cases}
\end{aligned}
$$

Dies ist die bekannte Lösungsdarstellung für die Ausbreitung einer ebenen Welle mit longitudinalem Amplitudenprofil $\propto \exp (-\bar{\mu} z)$ [38]. Die Lösung ist zu allen Zeiten positiv, d.h. die ebene Welle enthält keine Zuganteile. Da im eindimensionalen Grenzfall keine Beugung auftritt, entfällt die Ursache für das Entstehen von Zuganteilen. Für einen Beobachter im Punkt $q_{z}$ treffen Wellenanteile aus $z>q_{z}$ mit negativer Laufrichtung und $z<q_{z}$ mit positiver Laufrichtung zusammen, was zu den jeweils zwei Exponentialtermen führt. $\mathrm{Ab}$ dem Zeitpunkt $t=q_{z} / c_{0}$ erreichen den Beobachter aus Richtung $z<q_{z}$ die an der Quarz-Wasser-Grenzfläche reflektierten und dabei um $R_{\mathrm{Qw}}$ (3.19) abgeschwächten Wellenanteile. Im Ursprung ist insbesondere

$$
\left.p_{\delta, n}(\vec{o}, t)\right|_{w \rightarrow \infty}=\frac{\bar{\mu} n \Gamma_{n-1}\left(H_{0}\right)^{n}}{2}\left(1+\left(R_{\mathrm{QW}}\right)\right)^{n} \mathrm{e}^{-\bar{\mu} n r} .
$$




\subsubsection{Schallentwicklung in der Eintrittsebene}

Anders als auf der Strahlachse läßt sich die Poisson'sche Formel für einen beliebigen Punkt $\vec{q}=\left(q_{x}, q_{y}, 0\right)$ der Eintrittsebene $z=0$ nicht analytisch lösen: Das Gauß-Profil ist im inneren KS der Integrationssphäre um den Betrag $|\vec{q}|=\sqrt{q_{x}^{2}+q_{y}^{2}}$ vom Sphärenmittelpunkt verschoben und damit für die Integration nicht mehr radialsymmetrisch. Zur Bestimmung des Schallfeldes muß die Poisson'sche Formel dennoch nicht für jeden Punkt der Eintrittsebene numerisch gelöst werden, denn unabhängig von der integrationsbezogenen Verschiebung erzeugt das radialsymmetrische Gauß-Profil ein radialsymmetrisches Schallfeld. Dieses ist vollständig bestimmt, wenn die Poisson'sche Formel entlang einer Koordinatenachse ausgewertet wird (Radialschnitt).

Das äußere KS läßt sich ohne Einschränkung der Allgemeinheit so drehen, daß der Abstand des Beobachters $\vec{q}$ zur Integrationssphäre allein durch $q_{x}$ beschrieben wird $\left(q_{y}=0\right)$. Mit dieser Wahl des KS verhält sich das Gauß-Profil

$$
\left.H(r, \vartheta, \varphi)\right|_{q_{x}}=H_{0} \exp \left(-\frac{2}{w^{2}}\left(r^{2} \sin ^{2}(\vartheta)+2 r q_{x} \sin (\vartheta) \cos (\varphi)+q_{x}^{2}\right)\right)
$$

im inneren KS der Integrationssphäre symmetrisch bezüglich $\varphi \in[0, \pi]$ und $\varphi \in[\pi, 2 \pi]$, was zu einer Reduktion der Integration über $\varphi$ gemäß

$$
\left.\int_{0}^{2 \pi} f_{n}(r, \vartheta, \varphi)\right|_{q_{x}} \mathrm{~d} \varphi=\left.2 \int_{0}^{\pi} f_{n}(r, \vartheta, \varphi)\right|_{q_{x}} \mathrm{~d} \varphi
$$

führt.

Eine spezielle Symmetrie der Eintrittsebene $\left(q_{z}=0\right)$ besteht darin, daß sich der initiale und der gespiegelte Quelltermanteil aus Gl. (3.26) nur im Reflexionskoeffizienten $\left(R_{\mathrm{QW}}\right)^{n}$ unterscheiden:

$$
\left.f_{n}(r, \vartheta, \varphi)\right|_{q_{x}}=\left.n H^{n}(r, \vartheta, \varphi)\right|_{q_{x}} \cdot \begin{cases}\exp (-\bar{\mu} n(r \cos (\vartheta)) & : \quad r \cos (\vartheta) \geq 0 \\ \left(R_{\mathrm{QW}}\right)^{n} \exp (+\bar{\mu} n(r \cos (\vartheta)) & : \quad r \cos (\vartheta)<0\end{cases}
$$

Die Integranden $f_{n}$ verhalten sich hierdurch insgesamt symmetrisch bezüglich $\vartheta \in[0, \pi / 2]$ und $\vartheta \in[\pi / 2, \pi]$, so daß sich die Integration über $\vartheta$ ebenfalls reduziert:

$$
\left.\int_{0}^{\pi} f_{n}(r, \vartheta, \varphi)\right|_{q_{x}} \mathrm{~d} \vartheta=\left.\left(1+\left(R_{\mathrm{QW}}\right)^{n}\right) \int_{0}^{\pi / 2} f_{n}(r, \vartheta, \varphi)\right|_{q_{x}} \mathrm{~d} \vartheta
$$


Ausgehend von Gl. (3.24) ist die numerisch zu lösende Poisson'sche Formel damit von der Form:

$$
\begin{aligned}
p_{\delta, n}\left(q_{x}, t\right)= & \frac{\bar{\mu} n \Gamma_{n-1}\left(H_{0}\right)^{n}}{2 \pi}\left(1+\left(R_{\mathrm{QW}}\right)^{n}\right) \exp \left(-2 n \frac{q_{x}^{2}}{w^{2}}\right) . \\
& \frac{\partial}{\partial t}\left[t \int_{0}^{\pi / 2} \sin (\vartheta) \exp (-\bar{\mu} n r \cos (\vartheta)) \cdot\right. \\
& \left.\int_{0}^{\pi} \exp \left(-\frac{2 n r}{w^{2}}\left(r \sin ^{2}(\vartheta)+2 q_{x} \sin (\vartheta) \cos (\varphi)\right)\right) \mathrm{d} \varphi \mathrm{d} \vartheta\right] .
\end{aligned}
$$

Zur Berechnung der Integrale wurden die Integrationsalgorithmen von „Mathematica“ (Wolfram Research, Version 4.0) verwendet. Die Ergebnisse wurden durch Betrachtung der Lösungskonvergenz bei sukzessiver Erhöhung der einstellbaren Integrationsgenauigkeit überprüft. Die nachfolgende Zeitableitung wurde durch einfache Differenzenbildung in Mathematica realisiert und auch hier die Lösungskonvergenz bei sukzessiver Verkleinerung der Zeitschritte betrachtet. Eine weitere Lösungskontrolle erfolgte am Ursprung $\vec{o}$ durch den exemplarischen Vergleich mit den analytischen Lösungen $p_{\delta, n}(\vec{o}, t)$ aus Gl. (3.58). Die Ergebnisse der Berechnungen werden in Kapitel 4 dargestellt und diskutiert.

\subsection{Berechnung zum fasergeführten Laserstrahl}

Zur Beschreibung der Bestrahlungsgeometrie und Schallentwicklung am eingetauchten Faserende sei das äußere KS wie in Abbildung 3.4 gewählt. Bestehende Arbeiten haben sich mit der radialen Schallfeldentwicklung im Fernfeld [25, 67] oder mit der Gesamtentwicklung des Schallfeldes in der Ebene $y=0$ befaßt [6]. In allen Arbeiten wurde das Schallfeld sowohl im Experiment als auch im Modell auf Grundlage der Poisson'schen Formel untersucht. Die Experimente erfolgten jeweils an wässrigen Farbstofflösungen, die mit gepulsten Lasersystemen im sichtbaren Wellenlängenbereich bestrahlt wurden. Mögliche thermische Nichtlinearitäten wurden bei den zugehörigen Modellierungen nicht berücksichtigt. Im Rahmen dieser Arbeit wird die Schallentwicklung ausgehend von der Eintrittsebene $z=0$ diskutiert. Wie sie zu berechnen ist, wird im folgenden Unterabschnitten beschrieben. 


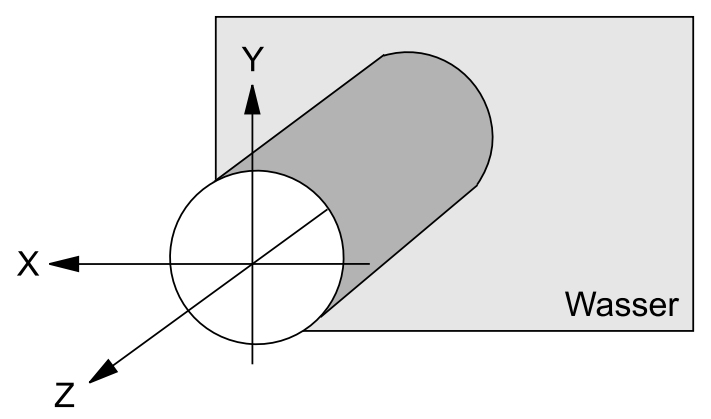

Abb. 3.4: Wahl des äußeren Koordinatensystems am eingetauchten Faserende.

\subsubsection{Schallentwicklung in der Eintrittsebene}

Das Strahlprofil des fasergeführten Laserstrahls sei über die Faserfläche mit Radius $a$ durch eine homogene Bestrahlung dargestellt, die zu den Seiten mit der Steigung $d$ gaußförmig abfällt:

$$
H(x, y)=H_{0} \cdot \begin{cases}1 & : \sqrt{x^{2}+y^{2}} \leq a \\ \exp \left(-\frac{\left(\sqrt{\left(x^{2}+y^{2}\right)}-a\right)^{2}}{d^{2}}\right): & \sqrt{x^{2}+y^{2}}>a\end{cases}
$$

Hierbei ist

$$
H_{0}=\frac{E}{\pi\left(a^{2}+d^{2}\right)},
$$

um für einen Laserpuls der Energie $E$ die Normierungsbedingung (3.43),

$$
\int_{-\infty}^{\infty} \int_{-\infty}^{\infty} H(x, y) \mathrm{d} x \mathrm{~d} y=E
$$

zu erfüllen. Eine Verspeckelung des Strahlprofils bleibt in der Darstellung (3.66) des Strahlprofils unberücksichtigt. In Abschnitt 4.3 wird gezeigt, daß diese Vernachlässigung in Bezug auf die Schallentwicklung gerechtfertigt ist.

Nach Übergang ins innere KS der Integrationssphäre ist das Faserstrahlprofil von der Form

$$
\begin{gathered}
\left.H(r, \vartheta, \varphi)\right|_{q_{x}}=H_{0} \cdot\left\{\begin{array}{ll}
1 & :\left.S(r, \vartheta, \varphi)\right|_{q_{x}} \leq a \\
\exp \left(-\frac{\left(S(r, \vartheta, \varphi) \mid q_{x}-a\right)^{2}}{d^{2}}\right) & :\left.S(r, \vartheta, \varphi)\right|_{q_{x}}>a
\end{array},\right. \text { mit } \\
\left.S(r, \vartheta, \varphi)\right|_{q_{x}}=\sqrt{r^{2} \sin ^{2}(\vartheta)+2 r q_{x} \sin (\vartheta) \cos (\varphi)+q_{x}^{2}}
\end{gathered}
$$

In dieser Darstellung ist die freie Drehbarkeit des äußeren KS berücksichtigt, wodurch der Abstand der Integrationssphäre zum Beobachter allein durch $q_{x}$ beschrieben werden kann 
$\left(q_{y}=0\right)$. Der vollständige Integrand ergibt sich durch Einsetzen des Faserstrahlprofils in Gl. (3.63),

$$
\left.f_{n}(r, \vartheta, \varphi)\right|_{q_{x}}=\left.n H^{n}(r, \vartheta, \varphi)\right|_{q_{x}} \cdot \begin{cases}\exp (-\bar{\mu} n(r \cos (\vartheta)) & : \quad r \cos (\vartheta) \geq 0 \\ \left(R_{\mathrm{QW}}\right)^{n} \exp (+\bar{\mu} n(r \cos (\vartheta)) & : \quad r \cos (\vartheta)<0\end{cases}
$$

Unter Ausnutzung der Symmetrien (3.61), (3.64) bzgl. $\varphi$ und $\vartheta$ führt er auf eine Poisson'sche Formel von der Gestalt

$$
\begin{aligned}
& p_{\delta, n}\left(q_{x}, t\right)=\frac{\left(1+\left(R_{\mathrm{QW}}\right)^{n}\right) \bar{\mu} n \Gamma_{n-1}\left(H_{0}\right)^{n}}{2 \pi} \frac{\partial}{\partial t}\left[t \int_{0}^{\pi / 2} \sin (\vartheta) \exp (-\bar{\mu} n r \cos (\vartheta)) .\right. \\
& \begin{cases}\int_{0}^{\pi} 1 \mathrm{~d} \varphi \mathrm{d} \vartheta & :\left.S(r, \vartheta, \varphi)\right|_{q_{x}} \leq a \\
\int_{0}^{\pi} \exp \left(-\frac{n}{d^{2}}\left(\left.S(r, \vartheta, \varphi)\right|_{q_{x}}-a\right)^{2}\right) \mathrm{d} \varphi \mathrm{d} \vartheta & :\left.S(r, \vartheta, \varphi)\right|_{q_{x}}>a\end{cases}
\end{aligned}
$$

Der obere Zweig der Poisson'schen Formel zum homogenen Strahlprofilanteil kann unmittelbar zu

$$
p_{\delta, n}\left(q_{x}, t\right)=\frac{\bar{\mu} n \Gamma_{n-1}\left(H_{0}\right)^{n}}{2 \pi}\left(1+\left(R_{\mathrm{QW}}\right)^{n}\right) \exp (-\bar{\mu} n r)
$$

aufgelöst werden. Die zugehörige Bedingung $S \leq a$ ist insbesondere für alle Radien $r \leq\left(a-q_{x}\right)$ erfüllt, sofern die Integration von einem Punkt innerhalb der Faserfläche ausgeht $\left(q_{x}<a\right)$.

Für den unteren Zweig der Poisson'schen Formel liegen die Gauß-Flanken des Faserstrahlprofils azentrisch zur Integrationssphäre. Analog zu den Überlegungen des vorigen Abschnittes muß die Schallentwicklung in diesem Fall numerisch bestimmt werden. Im Unterschied zum Gaußschen Laserstrahl gibt es hierbei auch im Ursprung $\vec{o}$ keine analytische Lösung, weil die Flanken dort ebenfalls verschoben liegen, und zwar gerade um den Faserradius $a$.

Für die Größe der Flankensteigung $d$ nennen G. Paltauf et al. [67] einen Verhältniswert $d / a \simeq 0.1$ zum Faserradius $a$. Dieser Wert basiert auf CCD-Aufnahmen einer optischen Zone, die sich über einen Faserradius $a=180 \mu \mathrm{m}$ und eine optische Eindringtiefe $\delta \simeq 12 \mu \mathrm{m}$ erstreckte. Da die Eindringtiefen zur Tm- und Ho-Laserwellenlänge in der Größenordnung 200-300 $\mu \mathrm{m}$ liegen (Tabelle 2.2), ist eine direkte Übertragung des Verhältnisses $d / a$ auf die Anregung mit diesen Lasern fraglich. Aus diesem Grund wurde mit 
Hilfe eines „ray tracing“-Programms (Solstis, Fa. Optis, Toulon/Frankreich) das Strahlprofil zur Tm- und Ho-Laserwellenlänge hinter Quarzfasern verschiedenen Durchmessers simuliert. Die verwendeten Simulationsparameter sind in Tabelle 3.3 aufgelistet.

Die Berechnungen bestätigten zunächst, daß die Flanken in guter Näherung durch eine Gaußkurve approximiert werden können (Abbildung 3.5). Weiter erwies sich die Flan-

\begin{tabular}{|c|c|c|c|c|}
\hline Anzahl Strahlen & Faserradien $[\mu \mathrm{m}]$ & Faser-NA & Wellenlängen $[\mathrm{nm}]$ & Auflösung $[\mu \mathrm{m}]$ \\
\hline $2 \cdot 10^{6}$ & 300,400 & 0.22 & 2010,2100 & 0.5 \\
\hline
\end{tabular}

Tabelle 3.3: Parameter zum ray tracing des Tm- und Ho-Laserstrahlprofils an einem Faserende.

kensteigung als unabhängig vom Faserradius, so daß eine Verhältnisangabe $a / d$ nicht reproduziert werden konnte. Statt dessen wurden als Mittelwerte über die jeweiligen Eindringtiefen Flankensteigungen von $25 \mu \mathrm{m}$ (Tm) und $45 \mu \mathrm{m}$ (Ho) ermittelt. Diese Werte wurden für die numerische Auswertung von Gl. (3.69) verwendet.

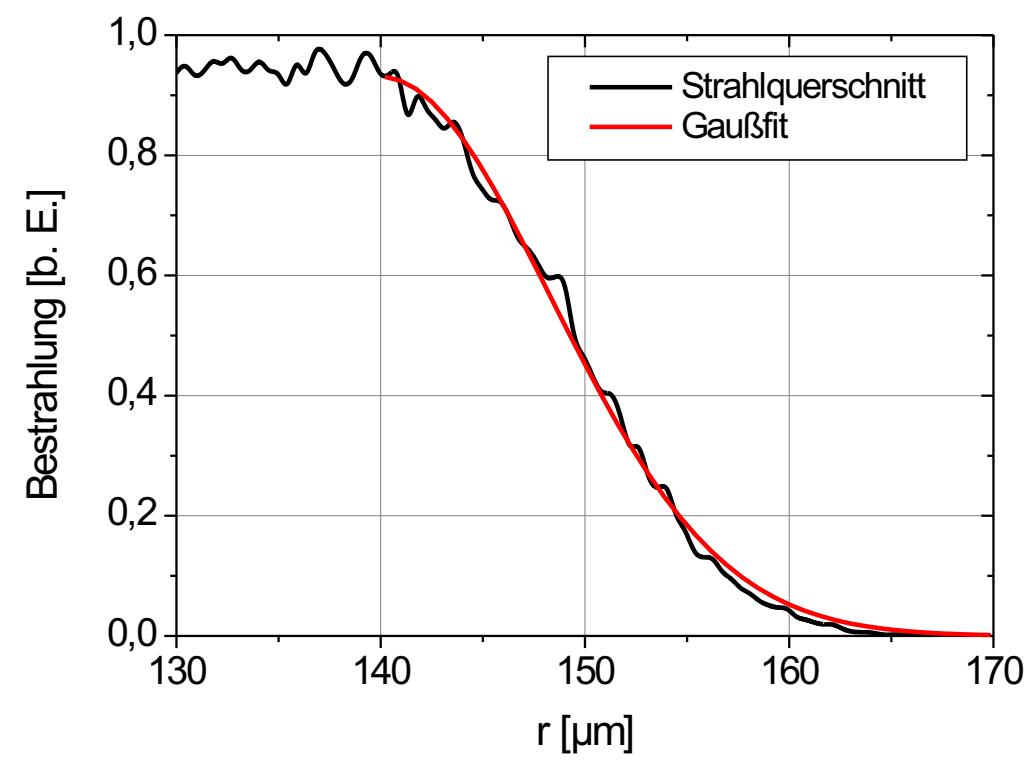

Abb. 3.5: Simulierter Strahlquerschnitt im Abstand $z=50 \mu \mathrm{m}$ hinter einer Faser mit Radius $a=$ $300 \mu \mathrm{m}$. Dazu angepaßte Gaußkurve mit Flankensteigung $d=18 \mu \mathrm{m}$. Das Rauschen im Strahlprofil resultiert aus der Berechnung einer endlichen Strahlanzahl (Tabelle 3.3) und stellt keine Verspeckelung dar. 


\section{Implikationen für den thermodynamischen Zustandsverlauf}

In den vorangegangenen Kapiteln wurden Modelle vorgestellt, aus denen sich die laserinduzierte Temperatur- und Schallentwicklung zu gegebenen Anregungsbedingungen ermitteln läßt. Die Anregungsbedingungen sind durch die Parameter Absorption, Strahlgeometrie (Gauß, Top-hat), Strahlradius, Pulsdauer und die applizierte Bestrahlung festgelegt. Je nach Wahl der Parameter nimmt der thermodynamische Zustand der angeregten Wasserprobe einen anderen Verlauf, was maßgeblichen Einfluß auf die nachfolgenden Ablationsvorgänge hat. Die Unterschiede im Zustandsverlauf sind im wesentlichen durch die Schallfeldentwicklung vermittelt, die in komplexer Weise von den Anregungsparametern abhängt, während die Termperatur stets eine monoton mit der Bestrahlung wachsende Größe ist.

Einleitend für die weiteren Betrachtungen wird in Abschnitt 4.1 eine Übersicht über die Schallfeldentwicklung zur Anregung mit dem Gaußschen Laserstrahl gegeben. Darauf aufbauend werden diejenigen Schallfeldgrößen herausgearbeitet, die charakteristisch für den Verlauf des thermodynamischen Zustandes der angeregten Wasserprobe sind. Im Unterabschnitt 4.1.2 wird die Verbindung zwischen Zustandsverlauf und Anregungsbedingungen hergestellt, indem die Abhängigkeit der charakteristischen Schallfeldgrößen von den o.g. Anregungsparametern untersucht wird. Das Kapitel schließt mit einer vergleichenden Betrachtung zur Schallentwicklung bei fasergeführter Laseranregung. 


\subsection{Schallentwicklung zum Gaußschen Laser- strahl}

Die Schallentwicklung zur Anregung mit dem Gaußschen Laserstrahl läßt sich beispielhaft am Verlauf in der Eintrittsebene $z=0$ darstellen. Dort werden die höchsten Bestrahlungen erreicht und damit auch die maximalen Temperaturen und Schallfeldamplituden. Die Maxima sind bestimmend für den möglichen Ablationsgrad und stehen daher im Fokus der weiteren Untersuchungen. Die wärmeleitungsbedingte und über die betrachteten Pulsdauern geringe axiale Verschiebung des Temperaturmaximums (Unterabschnitt 2.2.1) sei dabei vernachlässigt.

Zur Darstellung der Schallentwicklung in der Eintrittsebene sind in Abschnitt 3.3 die Impulsantworten $p_{\delta, n}\left(q_{x}, t\right)$ für die $n=1,2, \ldots, 8$ Reihenglieder der thermischen Nichtlinearität hergeleitet worden. Die Funktionen lösen die Poissonsche Formel entlang eines Radialschnittes (Variable $q_{x}$ ), aus dem das Schallfeld durch Rotation um die Strahlachse vervollständigt werden kann. Um eine erste Übersicht über den Lösungsverlauf zu erhalten, sei der Fall einer Anregung mit Bestrahlung $H \rightarrow 0$ angenommen, womit die Gesamtlösung allein durch die Impulsantwort $p_{\delta, 1}\left(q_{x}, t\right)$ zum linearen Quelltermanteil beschrieben wird.

Ein exemplarischer Verlauf dieser Lösung ist in Abbildung 4.1 dargestellt. Er wurde berechnet aus einer Anregungsgeometrie mit Radius $w=315 \mu \mathrm{m}$ und der Ruheabsorption $\mu\left(20^{\circ} \mathrm{C}\right)=6.48 / \mathrm{mm}$ der Tm-Laserwellenlänge. Er ist zum leichteren Vergleich für alle Graphen normiert aufgetragen. In den ersten Graphen ist zudem das Laserstrahlprofil eingezeichnet (rote Kurven). Ausgehend vom instantanen Energieeintrag bei $t=0 \mathrm{~ns}$ folgt das Schallfeld zunächst dem Profil der Anregung, beginnt aber bald, sich zu verbreitern und einen Ring auszubilden ( $t=85 \mathrm{~ns}$ ). Im Zentrum des Rings, das mit der Strahlmitte zusammenfällt, werden ab dem Zeitpunkt $t=95 \mathrm{~ns}$ die ersten Zuganteile des Schallfeldes beobachtet. Zudem bildet sich an dieser Stelle das Zugminimum des Schallfeldes aus ( $t=175 \mathrm{~ns}$ ). Im weiteren Verlauf entwickelt sich die initial gaußförmige Druckerhöhung zu einer bipolaren Welle, die sich radial in der Ebene ausbreitet. 

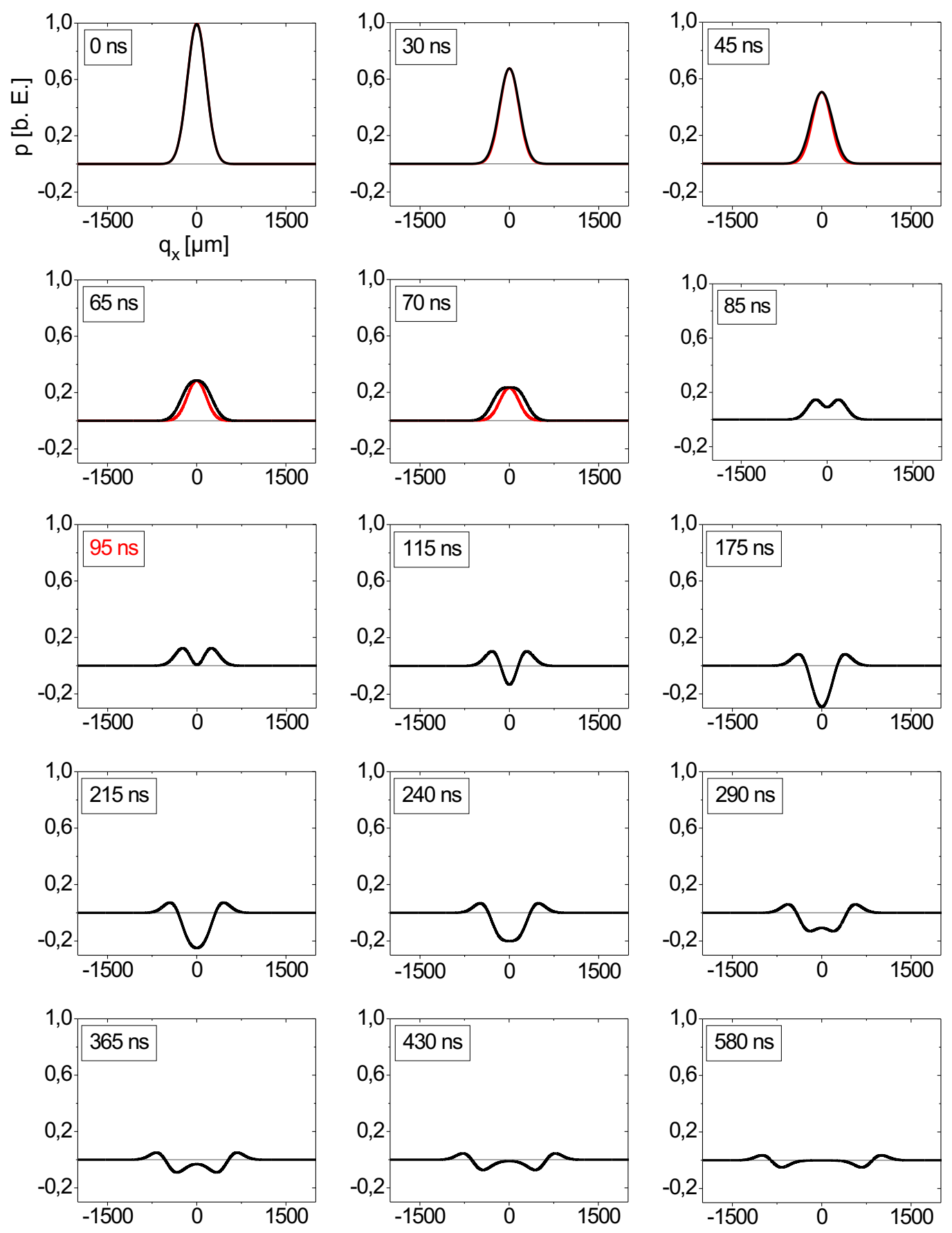

Abb. 4.1: Schallentwicklung in der Eintrittsebene (Radialschnitt) zu einer instantanen Anregung mit Radius $w=315 \mu \mathrm{m}$ und Absorption $\mu_{\mathrm{Tm}}\left(20^{\circ} \mathrm{C}\right)=6.48 / \mathrm{mm}$. Dazu in Rot das Gaußsche Strahlprofil der Laseranregung, jeweils auf das Maximum des Druckverlaufs skaliert. 

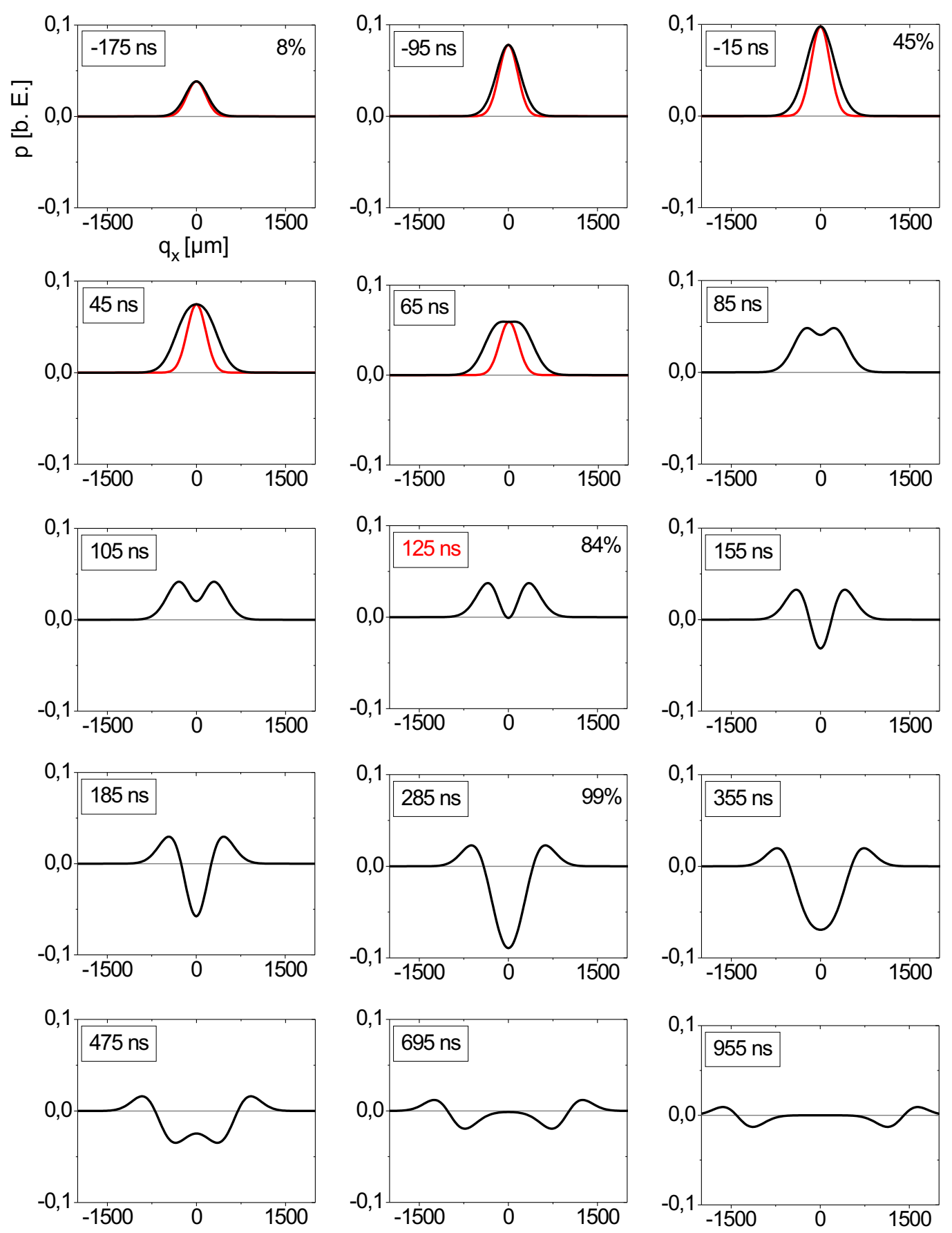

Abb. 4.2: Schallentwicklung in der Eintrittsebene (Radialschnitt) zu den Anregungsparametern $w=315 \mu \mathrm{m}, \mu=6.48 / \mathrm{mm}$ und $t_{\mathrm{p}}=300 \mathrm{~ns}$. Dazu in Rot das Gaußsche Strahlprofil der Laseranregung. Die in einigen Graphen genannten Prozentzahlen geben den bis zum Graphenzeitpunkt deponierten Energieanteil wieder. 
Ein ähnlicher Verlauf ergibt für die Schallentwicklung $p(\vec{q}, t) \equiv\left[p_{\delta, 1}(\vec{q}, \tau) \otimes g(\tau)\right](t) \mathrm{zu}$ einer zeitlich ausgedehnten Anregung $g(t)$ (3.38) (Abbildung 4.2): Bei der exemplarisch gewählten Pulsdauer $t_{\mathrm{p}}=300 \mathrm{~ns}$ ist aus dem Vergleich mit dem rot eingetragenen Laserstrahlprofil ist ersichtlich, daß sich das Schallfeld anfänglich mit der Anregung aufbaut, aber schon vor Erreichen des Druckmaximums bei $t=-15 \mathrm{~ns}$ davon abweicht. Aus der zunehmenden Verbreiterung entwickelt sich erneut ein Ring, in dessen Zentrum noch während der Anregung durch den Laserpuls die ersten Zuganteile beobachtet werden $(t=125 \mathrm{~ns})$. Ebenfalls im Zentrum findet sich das Zugminimum des Schallfeldes $t=285 \mathrm{~ns}$. Die weitere Entwicklung verläuft analog zur instantanen Energiedeponierung.

\section{Einfluß durch thermische Nichtlinearitäten}

Die dargestellten Verläufe haben gezeigt, daß sich für Bestrahlungen $H \rightarrow 0$ die maximalen Schallfeldamplituden sowie der Absolutbeginn der Zugphase stets in der Strahlmitte finden. $\mathrm{Ob}$ dies bei höheren Bestrahlungen $H$ weiterhin gilt, ist nicht unmittelbar einsichtig und bedarf daher der Überprüfung. Zu diesem Zweck wurden unter Hinzuziehung aller höheren Reihenglieder $p_{\delta, n}(\vec{q}, t)$ die Extrema und der Startzeitpunkt der Zugphase zum jeweiligen Radialabstand $q_{x}$ berechnet. Beispielhaft für die Anregungsparameter $w=315 \mu \mathrm{m}$ und $t_{\mathrm{p}}=300 \mathrm{~ns}$ aus Abbildung 4.2 ist in Abbildung 4.3 der Verlauf der Größen für eine Tm-Laseranregung mit Bestrahlung $H_{0}=164 \mathrm{~mJ} / \mathrm{mm}^{2}$ aufgetragen. Die Bestrahlung führt zu einer Endtemperatur in der Strahlmitte von $180^{\circ} \mathrm{C}$, der oberen Gültigkeitsgrenze der Funktion $\left.T(H)\right|_{\mu}$ aus Gl. (2.10). Die zugehörige mittlere Tm-Absorption berechnet sich mit Gl. (3.30) zu $\bar{\mu}=4.39 / \mathrm{mm}$. Aus den unteren Kurvenverläufen geht hervor, daß sich die Schallfeldextrema auch unter dem Einfluß thermischer Nichtlinearitäten im Zentrum ausbilden. Desgleichen zeigt der obere Kurvenverlauf, daß die ersten Zuganteile des Schallfeldes in der Strahlmitte zu finden sind.

Da die initiale Druckerhöhung direkt aus dem Temperaturanstieg folgt, dieser maximal in der Strahlmitte ist und der Grüneisenkoeffizient zudem im betrachteten Bestrahlungsbereich monoton mit der Temperatur wächst, ist die Ausbildung des Druckmaximums in der Strahlmitte nachvollziehbar. Für den beugungsbedingten Zuganteil gibt es keine vergleichbare Argumentation, Zugphasenbeginn und Zugmaximum mußten durch die numerische Auswertung der Impulsantwortfunktionen $p_{\delta, n}\left(q_{x}, t\right)$ lokalisiert werden. 


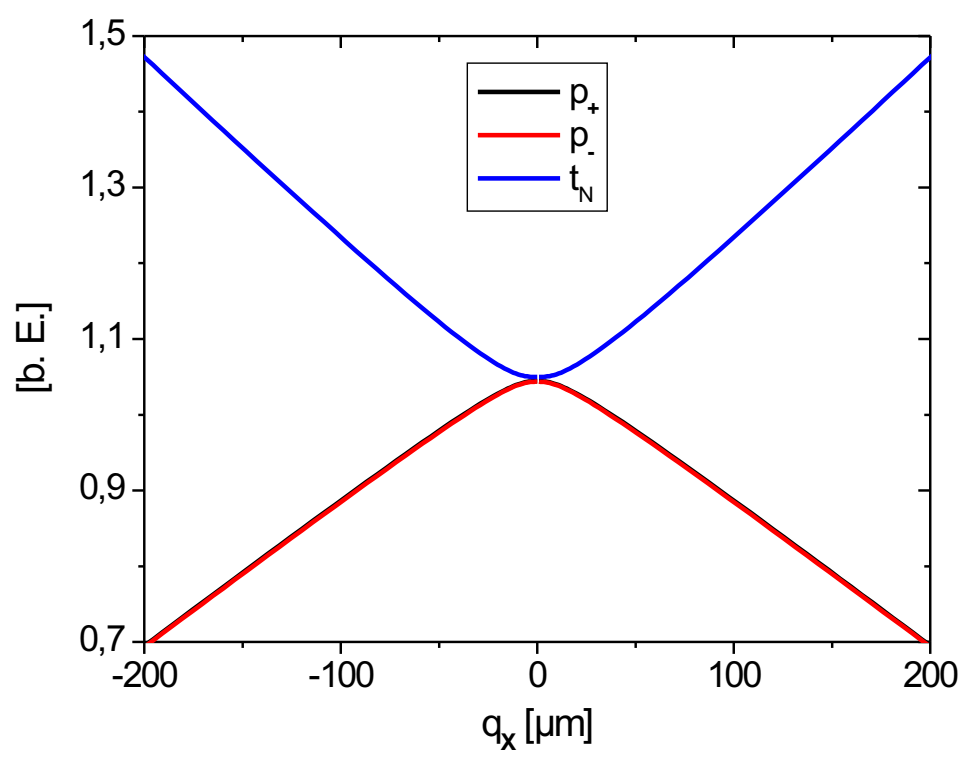

Abb. 4.3: Verlauf der Betragsmaxima von Druck $\left(p_{+}\right)$und Zug $\left(p_{-}\right)$sowie des Startzeitpunktes $t_{\mathrm{N}}$ der Zugphase zum jeweiligen Radialabstand $q_{x}$, bei einer Bestrahlung $H_{0}=164 \mathrm{~mJ} / \mathrm{mm}^{2}$ $\left(\bar{\mu}=4.39 / \mathrm{mm}, w=315 \mu \mathrm{m}\right.$ und $\left.t_{\mathrm{p}}=300 \mathrm{~ns}\right)$. Alle Größen sind auf den jeweiligen Wert bei $q_{x}=0 \mu \mathrm{m}$ normiert. Die Druck- und Zugkurven verlaufen praktisch gleich und sind deshalb nur schwer zu trennen.

\subsubsection{Phasendiagramm zur Anregung}

Das Zusammentreffen aller Extrema in der Strahlmitte ermöglicht es, die weiteren Betrachtungen auf diesen Punkt der Ebene $z=0$ zu konzentrieren. Er entspricht dem Koordinatenursprung $\vec{o}$ des in Abschnitt 3.2 definierten KS. Die Betrachtung der Schallentwicklung im Ursprung bietet den zusätzlichen Vorteil, daß zur Berechnung der Drucktransiente $p(\vec{o}, t)$ die analytischen Lösungsformeln $p_{\delta, n}(\vec{o}, t)$ gemäß Gl. (3.58) genutzt werden können. Zusammen mit Gl. (2.9),

$$
\left.T(t)\right|_{\mu}=\frac{1}{b}\left[\mu\left(t_{\mathrm{N}}\right) \exp \left(\frac{b}{\rho C} \int_{-\infty}^{t} I_{0}(\tau) \mathrm{d} \tau\right)-a\right]
$$

ist der zeitliche Verlauf von Druck und Temperatur über der Anregung bestimmbar. Für die Parameter der zu Abbildung 4.3 angenommenen Tm-Laseranregung folgt der in Abbildung 4.4 dargestellte Verlauf. 


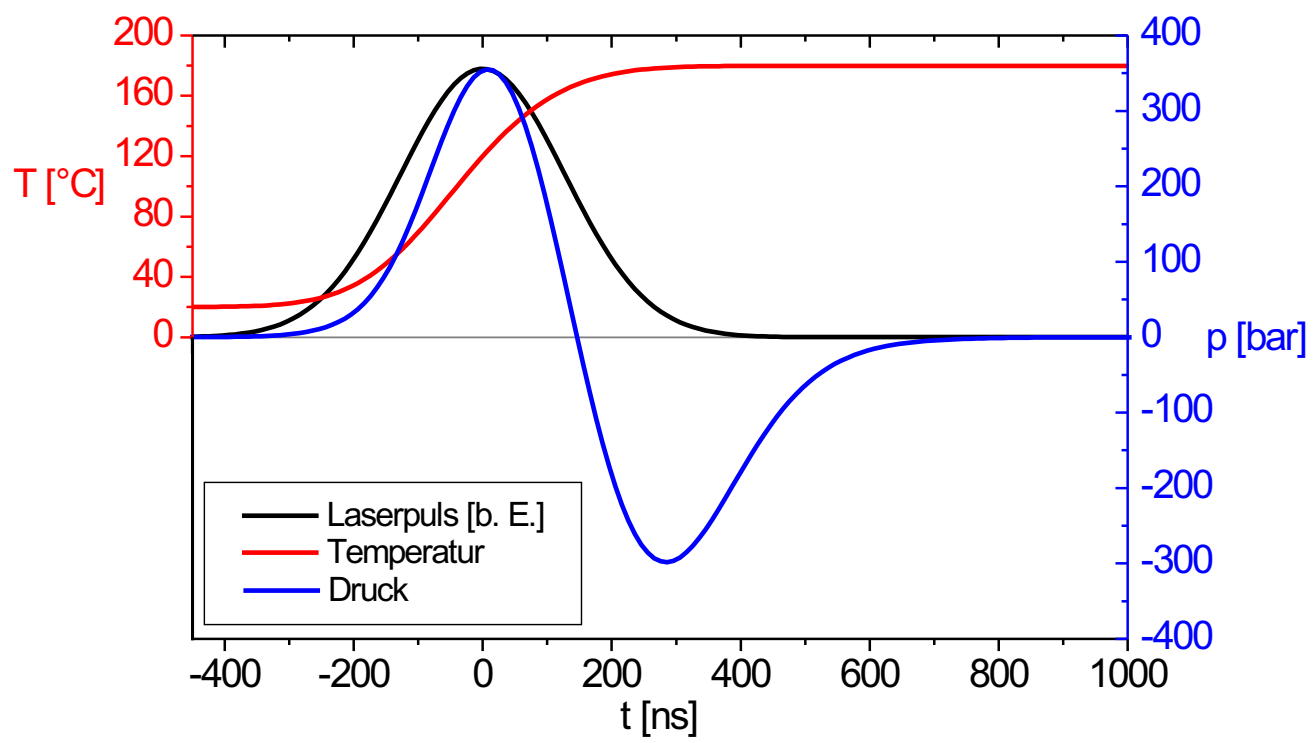

Abb. 4.4: Verlauf von Druck und Temperatur im Ursprung $\vec{o}$ für die Parameter einer TmLaseranregung mit $H_{0}=164 \mathrm{~mJ} / \mathrm{mm}^{2}, \bar{\mu}=4.39 / \mathrm{mm}, w=315 \mu \mathrm{m}$ und $t_{\mathrm{p}}=300 \mathrm{~ns}$.

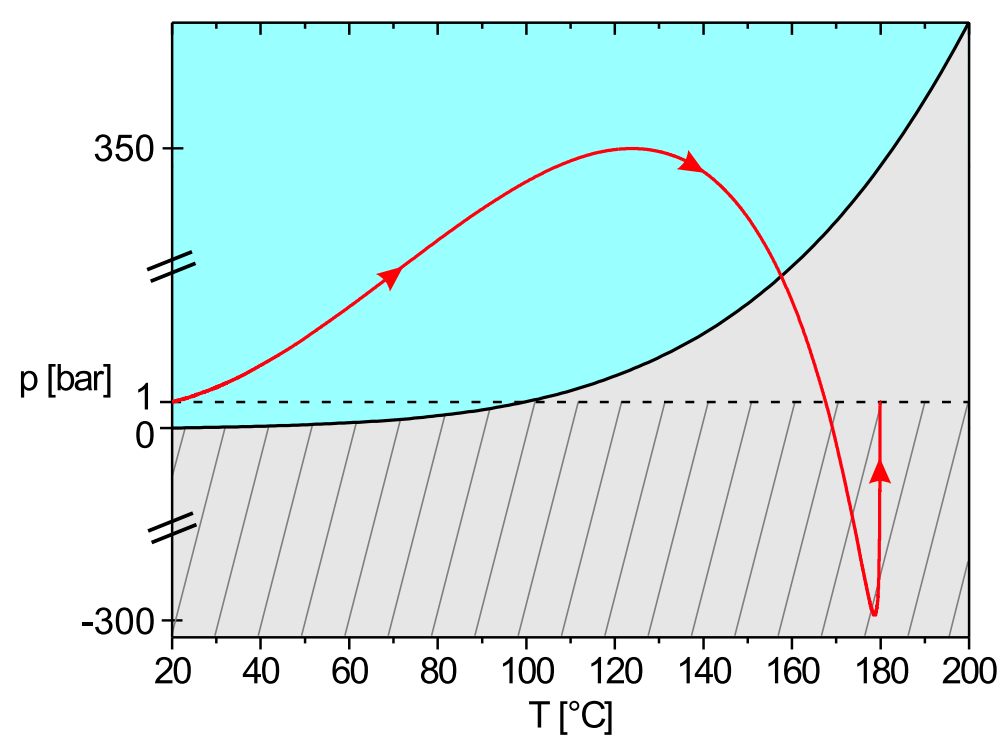

Abb. 4.5: Schematische Übertragung des Druck- und Temperaturverlaufs zur Tm-Laseranregung aus Abbildung 4.4 in einen thermodynamischen Zustandsverlauf (rote Kurve) im $p$ - $T$-Phasendiagramm. Der grau unterlegte Bereich markiert die Dampf-Phase, die durch die Dampfdruckkurve $p_{\mathrm{D}}(T)$ von der flüssigen Phase (blau unterlegt) getrennt ist. Die thermoelastische Zugphase ( $p<1$ bar) ist durch Schraffur hervorgehoben. 
Mit der gleichzeitigen Kenntnis von Druck und Temperatur läßt sich im $p-T$ Phasendiagramm nachzeichnen, welchen thermodynamischen Zustandsverlauf die angeregte Wasserprobe nimmt (Abbildung 4.5): Ausgehend vom Ruhezustand $p=1$ bar und $T=20^{\circ} \mathrm{C}$ steigen Temperatur und Druck zunächst an, wobei die thermoelastische Druckerhöhung so lange bestehen bleibt, daß der Übergang in die Dampf-Phase nicht beim normalen Siedepunkt von $100^{\circ} \mathrm{C}$ erfolgt, sondern bei einer höher liegenden Übergangstemperatur $T_{\breve{U}} \simeq 160^{\circ} \mathrm{C}$. Wenn beim Erreichen der Dampf-Phase unmittelbar die Verdampfung der Wasserprobe einsetzt, so endet der Zustandsverlauf an dieser Stelle. Anderenfalls geht die Probe entlang des markierten Pfades in einen metastabilen Überhitzungszustand über. Dies kann z.B. der Fall sein, wenn in der optischen Zone nur wenige, kleine Nukleationskeime vorliegen, deren erhöhte Oberflächenspannung dem Verdampfungsprozeß entgegenwirkt (Unterabschnitt 2.3.1). Mit fortschreitender Wirkung der Zugphase wird ein Aufrechterhalten des metastabilen Zustandes zunehmend unwahrscheinlicher. Sobald die Keime kavitationsbedingt aktiviert werden und im Vergrößern ihre Oberflächenspannung verringern, stehen geeignete Phasengrenzen für den Beginn der Verdampfung bereit.

Der Übergangstemperatur $T_{\ddot{U}}$ kommt aus Sicht der medizinischen Anwendung eine besondere Bedeutung zu. So definiert sie über den erreichten Überhitzungsgrad, ob die nachfolgende Verdampfung stetig oder explosiv verläuft. Dies legt wiederum fest, wieviel Gewebe pro Puls abgetragen wird. Zudem ist sie maßgebend für den Grad der Gewebeschädigung durch thermische Denaturierung. Der Betrag von $T_{\ddot{U}}$ hängt wesentlich davon ab, welchen Verlauf der Zustandspfad bis zum Schnittpunkt mit der Phasengrenze nimmt. Abhängigkeiten dieses Verlaufs von den Anregungsparametern $\left(w, \bar{\mu}, t_{\mathrm{p}}, H\right)$ werden durch die entsprechenden Abhängigkeiten des Schallfeldes vermittelt, weniger durch die monoton mit der Bestrahlung wachsende Temperatur. Als Beispiel hierfür sei eine Ho-Laseranregung zu den Anregungsparametern $H=296 \mathrm{~mJ} / \mathrm{mm}^{2}$ mit $\bar{\mu}=2.35 / \mathrm{mm}$, $w=600 \mu \mathrm{m}$ und $t_{\mathrm{p}}=300 \mathrm{~ns}$ betrachtet, die zu Verläufen für Druck, Temperatur und den Zustandspfad gemäß Abbildung 4.6 und 4.7 führt. 


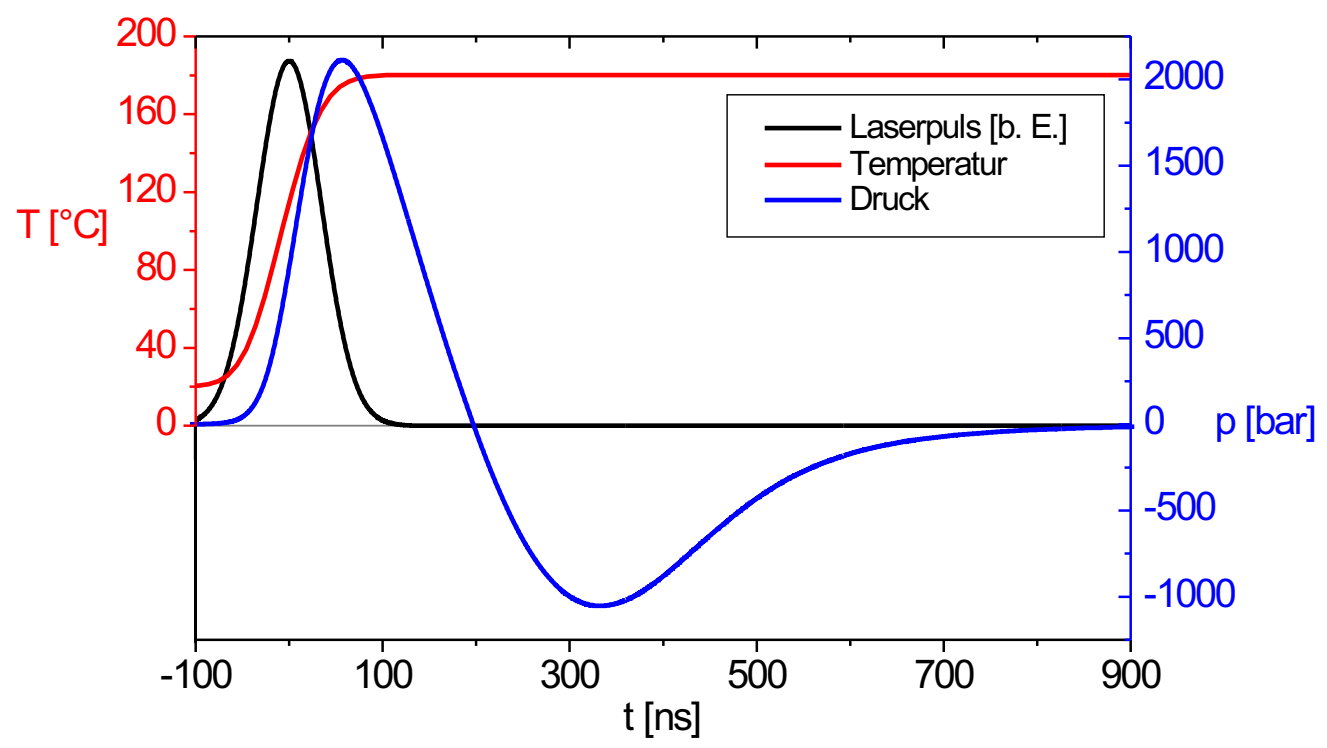

Abb. 4.6: Verlauf von Druck und Temperatur im Ursprung $\vec{o}$ für die Parameter einer HoLaseranregung mit $H_{0}=296 \mathrm{~mJ} / \mathrm{mm}^{2}, \bar{\mu}=2.35 / \mathrm{mm}, w=600 \mu \mathrm{m}$ und $t_{\mathrm{p}}=80 \mathrm{~ns}$.

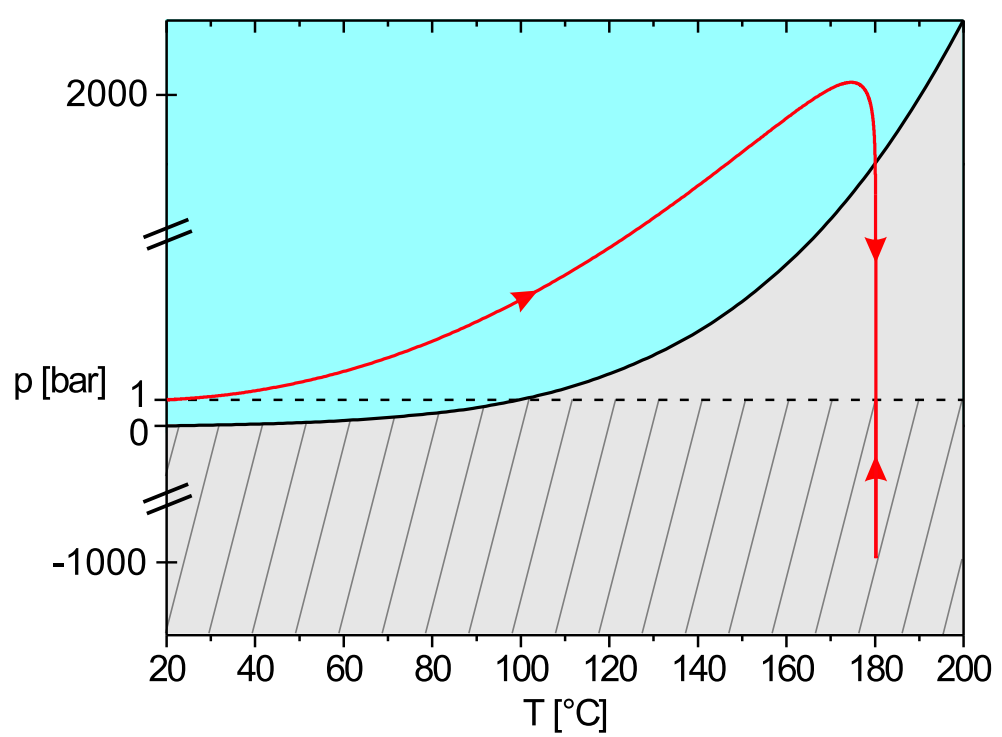

Abb. 4.7: Schematische Übertragung des Druck- und Temperaturverlaufs zur Ho-Laseranregung aus Abbildung 4.6 in den thermodynamischen Zustandsverlauf (rote Kurve) im $p$ - $T$ Phasendiagramm. Der grau unterlegte Bereich markiert die Dampf-Phase, die durch die Dampfdruckkurve $p_{\mathrm{D}}(T)$ von der flüssigen Phase (blau unterlegt) getrennt ist. Die thermoelastische Zugphase ( $p<1$ bar) ist durch Schraffur hervorgehoben. 
Die Bestrahlung ist so gewählt, daß dieselbe Endtemperatur wie zur vorherigen TmLaseranregung erreicht wird. Dadurch sind die schallfeldvermittelten Unterschiede im Zustandsverlauf unmittelbar ersichtlich. Die Ho-Laseranregung zeichnet sich durch eine kürzere Pulsdauer aus, bei zugleich größerer Eindringtiefe $1 / \bar{\mu}$ und einem größer gewählten Strahlradius. Das bedeutet sowohl verbesserte Bedingungen für den akustischen Einschluß als auch eine verminderte akustische Beugung, bedingt durch die größeren Abmessungen der optischen Zone. Zusammen ergibt sich hieraus ein ungestörter Druckaufbau über die gesamte Dauer der Anregung, wodurch der nachfolgende Druckabbau und der Übergang in die Zugphase zu einem Zeitpunkt stattfinden, bei dem bereits die Maximaltemperatur $T_{\max } \simeq 180^{\circ} \mathrm{C}$ vorherrscht. Im Zustandsverlauf führt dies zu einem Übergang in die Dampf-Phase, der ebenfalls bei der Maximaltemperatur erfolgt. Es ist $T_{\ddot{U}}=T_{\max }$ und damit der höchst mögliche Grad an Überhitzung erreicht. 


\subsubsection{Charakteristische Schallfeldgrößen}

Der erreichbare Überhitzungsgrad hängt in der Tendenz nur von wenigen charakteristischen Größen der Drucktransiente ab: So begünstigt zunächst eine hohe Druckamplitude

$$
p_{+} \equiv \max _{t}(p(\vec{o}, t))
$$

den Phasenübergang bei hohen Temperaturen und damit eine mögliche Überhitzung. Wie nah die Übergangstemperatur $T_{\ddot{U}}$ dabei an die Maximaltemperatur $T_{\max }$ heranreicht, ist durch diejenige Temperatur $T_{\mathrm{N}}$ festgelegt, die zum Zeitpunkt $t_{\mathrm{N}}$ des Nulldurchgangs zwischen Druck- und Zugphase erreicht ist. Im Zustandsverlauf korrespondiert der Nulldurchgang mit dem Rückgang auf 1 bar Umgebungsdruck, ab dem oberhalb von $100{ }^{\circ} \mathrm{C}$ der Übergang in die Dampf-Phase möglich ist. Es gilt also die Reihenfolge $T_{\ddot{U}} \leq T_{\mathrm{N}} \leq T_{\max }$, so daß eine maximale Überhitzung im Fall von $T_{\mathrm{N}}=T_{\max }$ erreicht wird. Diesen Fall zeigte das Beispiel zur Ho-Laseranregung (Abbildung 4.7).

Da der Abstand zwischen $T_{\mathrm{N}}$ und $T_{\max }$ von der Lage des Nulldurchgangs relativ zur Temperaturentwicklung $T(t)$ abhängt, ist es zweckmäßig, ihn durch eine relative Zeitgröße mit Bezug zum Temperaturverlauf zu charakterisieren. Der Quotient $t_{\mathrm{N}} / t_{\mathrm{p}}$ zur Pulsdauer $t_{\mathrm{p}}$ ist eine leicht zu bestimmende Größe, die einen Bezug zur applizierten Bestrahlung herstellt (Tabelle 4.1). Aufgrund des streng monotonen Wachstums der Temperatur mit der Bestrahlung ist damit implizit auch der gewünschte Bezug zum Temperaturverlauf hergestellt.

\begin{tabular}{c||c|c|c|c|c|c|}
$t_{\mathrm{N}} / t_{\mathrm{p}}$ & -1 & -0.5 & 0 & 0.5 & 1 & 1.5 \\
\hline$E_{\text {dep }}[\%]$ & 1 & 12 & 50 & 88 & 99 & 99.9
\end{tabular}

Tabelle 4.1: Deponierter Energieanteil $E_{\text {dep }}[\%]$ zum relativen Zeitpunkt $t_{\mathrm{N}} / t_{\mathrm{p}}$ in einem gaußförmigen Pulsprofil der Pulsbreite $t_{\mathrm{p}}$ (FWHM) - vgl. Abbildung 4.8.

Mit dem Eintritt in die Dampf-Phase bei der Übergangstemperatur $T_{\ddot{U}}$ nimmt zuletzt die Amplitude des thermoelastischen Zugphase Einfluß darauf, bei welcher Temperatur die überhitzte Probe tatsächlich verdampft. Im Fall hoher Zugamplituden ist eine Verdampfung nahe $T_{\ddot{U}}$ wahrscheinlich, anderenfalls kann sich die Verdampfung bis zum Erreichen der Maximaltemperatur verzögern oder findet überhaupt nicht statt. Charakteristisch für den Zustandsverlauf ist daher neben der Druckamplitude $p_{+}$und dem Nulldurchgangsquotienten $t_{\mathrm{N}} / t_{\mathrm{p}}$ auch die Zugamplitude

$$
p_{-} \equiv\left|\min _{t}(p(\vec{o}, t))\right|
$$




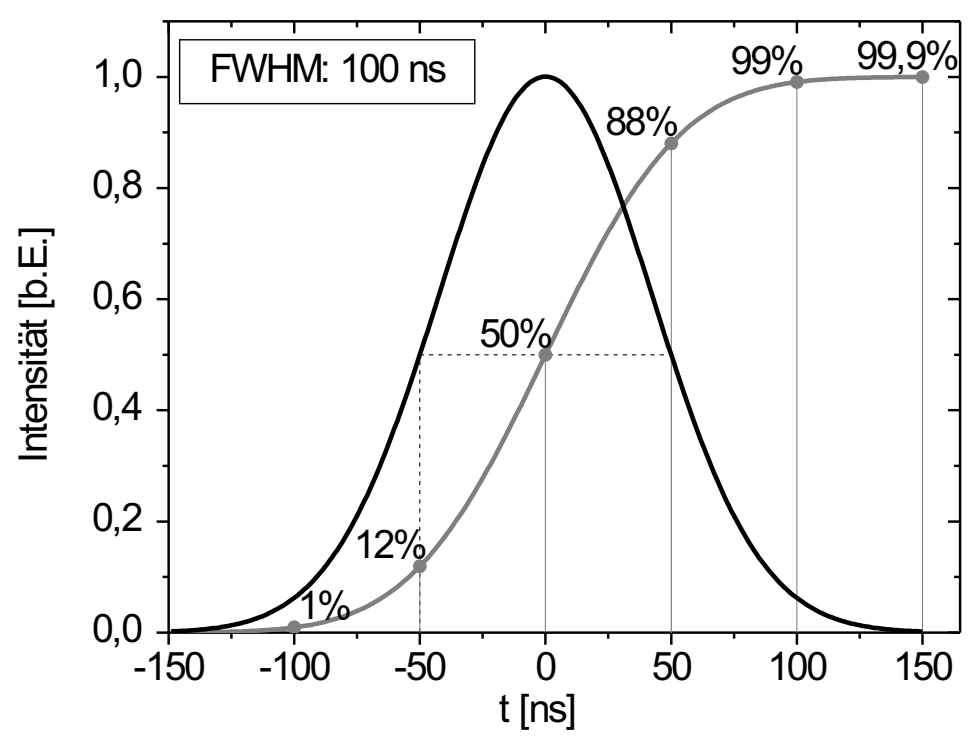

Abb. 4.8: Energieverteilung in einem gaußförmigen Laserpulsprofil, dargestellt am Beispiel eines Pulses der Dauer $t_{\mathrm{p}}=100 \mathrm{~ns}(\mathrm{FWHM})$.

\subsection{Abhängigkeit von den Anregungsparametern}

Im vorigen Abschnitt wurde demonstriert, wie Änderungen der thermoelastischen Schallentwicklung signifikante Änderungen im thermodynamischen Zustandsverlauf nach sich ziehen können. Gegenstand dieses Abschnittes ist es, die Abhängigkeit der charakteristischen Schallfeldgrößen von den Anregungsparametern zu untersuchen und damit eine Verbindung zwischen gewählter Anregung und resultierendem Zustandsverlauf herzustellen.

Die Bedingungen zu einer Gaußschen Laseranregung seien durch den Strahlradius $w$, die mittlere Absorption $\bar{\mu}$, die Pulsdauer $t_{\mathrm{p}}$ und die Pulsenergie $E$ festgelegt. In der Anwendung sind von diesen Parametern die Energie und der Strahlradius variabel, während die Pulsdauer durch das verwendete Lasersystem vorgegeben ist und die mittlere Absorption sich aus der applizierten Bestrahlung $H_{0}=2 E /\left(\pi w^{2}\right)$ errechnet. Im Grundsatz sind daher Abhängigkeiten vom Typ $\left.f(w, E)\right|_{\bar{\mu}, t_{\mathrm{p}}}$ zu untersuchen, in denen Absorption und Pulsdauer als diskrete Parameter auftreten. Die Größe $f$ steht stellvertretend für eine der charakteristischen Schallfeldgrößen $p_{+}, p_{-}$oder $t_{\mathrm{N}} / t_{\mathrm{p}}$.

Die Abhängigkeit vom Strahlradius wird nachfolgend für Werte zwischen $w=2 \mu \mathrm{m}$ und $w \leq 5 \mathrm{~mm}$ untersucht. Die Untergrenze entspricht der Größe der betrachteten 
Laserwellenlängen und beschreibt die minimale Periode der Speckelmodulationen am Austritt einer Multimodefaser. Mit der gewählten Obergrenze wird der Bereich typischer experimenteller Strahlradien abgedeckt. Als Pulsdauern werden die Werte $30 \mathrm{~ns}$ und $200 \mathrm{~ns}$ für den Ho-Laser bzw. $200 \mathrm{~ns}$ und $500 \mathrm{~ns}$ für den Tm-Laser betrachtet, entsprechend den in der Literatur genannten Unter- und Obergrenzen (Abschnitt 2.1). Dazu werden für die Pulsenergie Werte von $25 \mathrm{~mJ}, 50 \mathrm{~mJ}$ und $100 \mathrm{~mJ}$ eingesetzt. Die Ergebnisdarstellung vereinfacht sich so auf Kurvenscharen vom Typ $\left.f(w)\right|_{\bar{\mu}, t_{\mathrm{p}}, E}$.

Neben den Verläufen zu konstanter Pulsenergie werden die Verläufe $\left.f(w)\right|_{\bar{\mu}, t_{\mathrm{p}}, \Delta T}$ zu einer konstanten Erhitzung um den Betrag $\Delta T$ untersucht. Unter Annahme einer konstanten Erhitzung zeigen sich die Änderungen der charakteristischen Schallfeldgrößen bei gleichbleibenden Voraussetzungen für die Verdampfung und die thermische Denaturierung. Für eine gegebene Wellenlänge wird eine konstante Erhitzung durch eine konstante Bestrahlung erzielt. Bei Variation des Strahlradius erfordert dies eine entsprechende Anpassung der Pulsenergie. Nachfolgend werden die Verläufe zu den Erhitzungsgraden $\Delta T=40^{\circ} \mathrm{C}$, $80^{\circ} \mathrm{C}$ und $160^{\circ} \mathrm{C}$ untersucht. Ausgehend von einer Ruhetemperatur $T_{0}=20^{\circ} \mathrm{C}$ sind so insbesondere die Erhitzung auf den normalen Siedepunkt $T=100^{\circ} \mathrm{C}$ dargestellt sowie eine beispielhafte Überhitzung der Wasserprobe.

\subsubsection{Betrachtung bei konstanter Erhitzung}

\section{Druckamplitude}

Die Verläufe $\left.p_{+}(w)\right|_{\bar{\mu}, t_{\mathrm{p}}, \Delta T}$ der Druckamplitude sind in Abbildung 4.9 zusammengestellt. Die beiden oberen Graphen zeigen den Verlauf zur Tm-, die unteren zur HoLaseranregung. Der Unterschied zwischen beiden Anregungen besteht rechnerisch in der Wahl der zugehörigen mittleren Absorptionen. Aus dem Vergleich des Tm- und HoGraphen zu derselben Pulsdauer $t_{\mathrm{p}}=200 \mathrm{~ns}$ läßt sich daher eine Aussage über die Änderung der Druckamplitude mit der Absorption gewinnen. Die Änderungen mit der erzielten Temperaturerhöhung können durch direkten Kurvenvergleich innerhalb eines Graphen abgelesen werden und die Änderungen mit der Pulsdauer ergeben sich aus dem graphenübergreifenden Vergleich zu jeweils einer Laseranregung.

Die Kurven $p_{+}(w)$ verlaufen qualitativ für alle Parameterkombinationen $\left(\bar{\mu}, t_{\mathrm{p}}, \Delta T\right)$ gleich: Ausgehend vom kleinsten betrachteten Radius $w=2 \mu \mathrm{m}$ sind die Kurven streng monoton wachsend und laufen asymptotisch gegen einen oberen Grenzwert. Zu einer gegebenen Erhitzung werden die höchsten Amplituden bei kurzen Pulsdauern und niedrigen 

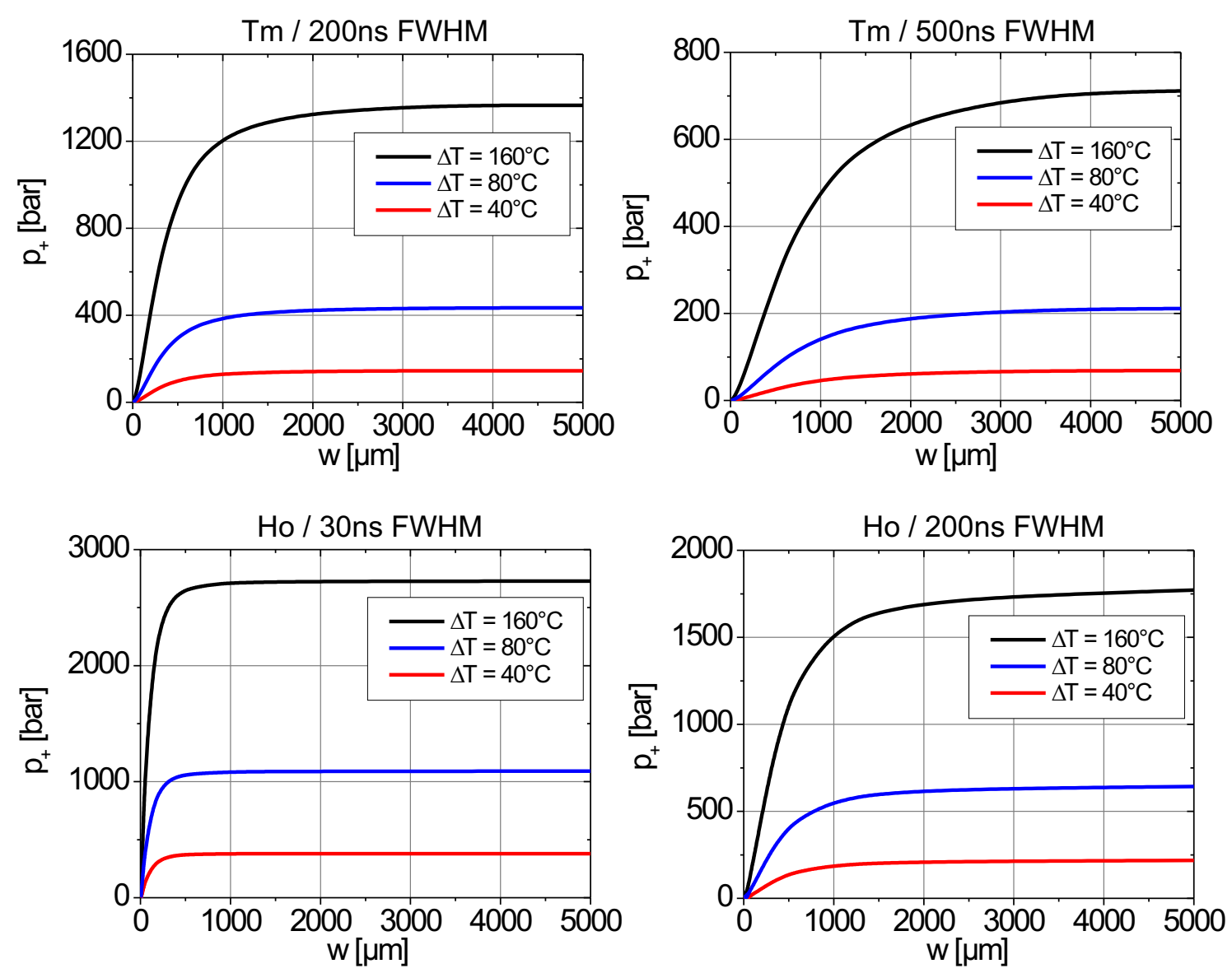

Abb. 4.9: Verlauf der Funktion $\left.p_{+}(w)\right|_{\bar{\mu}, t_{\mathrm{p}}, \Delta T}$ zur Tm- und Ho-Laseranregung mit den angegebenen Pulsdauern. Man beachte die unterschiedliche Skalierung der Ordinaten. Die mittleren Absorptionen berechnen sich aus den genannten Temperaturerhöhungen zu $\mu_{\mathrm{Tm}}=5.94 / \mathrm{mm}\left(40^{\circ} \mathrm{C}\right), 5.43 / \mathrm{mm}\left(80^{\circ} \mathrm{C}\right), 4.39 / \mathrm{mm}\left(160^{\circ} \mathrm{C}\right)$, sowie $\mu_{\text {но }}=2.97 / \mathrm{mm}$ $\left(40^{\circ} \mathrm{C}\right), 2.77 / \mathrm{mm}\left(80^{\circ} \mathrm{C}\right), 2.35 / \mathrm{mm}\left(160^{\circ} \mathrm{C}\right)$.

Absorptionen gefunden. Bei Betrachtung der Amplituden bei festem Strahlradius zeigt sich ein überproportionales Wachstum der Amplitude mit dem Erhitzungsgrad. Insgesamt weisen die Amplituden für alle Pulsdauern und Absorptionen Beträge auf, die ein hohes Überhitzungspotenzial erwarten lassen. So ist der zur Erhitzung $\Delta(T)=160^{\circ} \mathrm{C}$ zugehörige Dampfdruck $p_{\mathrm{D}} \simeq 10$ bar in allen Kurven spätestens ab einem Strahlradius von $50 \mu \mathrm{m}$ erreicht (Dampfdruckkurve $p_{\mathrm{D}}(T)$ in Abbildung 2.8). 


\section{Diskussion der Verlaufsmerkmale}

Die dargestellten Verläufe berechnen sich aus den zugehörigen Lösungsfunktionen (3.58),

$$
p_{\delta, n}(\vec{o}, t)=\frac{\left(1+\left(R_{\mathrm{QW}}\right)^{n}\right) \bar{\mu} n \Gamma_{n-1}\left(H_{0}\right)^{n}}{2} \cdot \hat{p}_{\delta, n}(\vec{o}, t),
$$

mit

$$
\hat{p}_{\delta, n}(\vec{o}, t) \equiv\left[N(r, n)\left\{\operatorname{erfi}(a(r, n))-\operatorname{erfi}\left(\bar{\mu} w \sqrt{\frac{n}{8}}\right)\right\}+\mathrm{e}^{-\bar{\mu} n r}\right]
$$

und $N(r, n)$ gemäß Gl. (3.54). Unter der getroffenen Annahme eines konstanten Erhitzungsgrades ist der Vorfaktor eine konstante Größe, da auch Bestrahlung und Absorption unverändert bleiben. Die gefundenen Verlaufsmerkmale sind damit allein vom Verhalten des normierten Lösungsanteils $\hat{p}_{\delta, n}(\vec{o}, t)$ bestimmt. Dieser spiegelt die Schallfeldabhängigkeiten von der Bestrahlungsgeometrie wider, d.h. insbesondere von den zugehörigen akustischen Einschlußbedingungen (2.14)

$$
M_{\mathrm{AE}}=\frac{1}{t_{\mathrm{p}}} \frac{D}{c_{0}} .
$$

Hierbei ist $D=\min (2 w, 1 / \bar{\mu})$ die charakteristische Länge der optischen Zone und $c_{0}$ die Schallgeschwindigkeit. Ausgehend von $w \simeq 0$ verbessert ein zunehmender Strahlradius den akustischen Einschluß so lange, bis die optischen Eindringtiefe $1 / \bar{\mu}$ bestimmend für die Einschlußbedingungen wird. Eine weitere Vergrößerung des Strahlradius führt zu keiner weiteren Zunahme der Druckamplitude, die Kurve nähert sich dem asymptotischen Grenzwert. Im Vergleich der Kurven zu gleicher Erhitzung, aber unterschiedlicher Pulsdauer und Absorption ist der höchste akustische Einschluß erreicht, wenn $t_{\mathrm{p}}$ und $\bar{\mu}$ minimal sind.

An dieser Stelle sei betont, daß die Größe $M_{\mathrm{AE}}$ lediglich als Indikator für die geometrischen Randbedingungen der Schallentwicklung dient und keine Rückschlüsse auf den genauen Kurvenverlauf $\left.p_{+}(w)\right|_{\bar{\mu}, t_{\mathrm{p}}, \Delta T}$ zuläßt. Insbesondere liefert der Wechselpunkt $w=(1 / 2 \bar{\mu})$ der charakteristischen Länge $D$ keinen Anhaltspunkt zum asymptotischen Übergang der Kurvenverläufe.

\section{Zugamplitude}

Die Verläufe $\left.p_{-}(w)\right|_{\bar{\mu}, t_{\mathrm{p}}, \Delta T}$ zum Betrag der Zugamplitude sind in Abbildung $4.10 \mathrm{zu}-$ sammengefaßt. Die Kurven zeigen einen im Grundsatz einheitlichen Verlauf, der vom Ursprung $(0,0)$ ausgeht, in ein lokales Maximum übergeht und für große Strahlradien 
wieder gegen Null strebt. Die höchsten Zugamplituden werden analog zum Druck bei kurzen Pulsdauern, niedrigen Absorptionen und hohen Erhitzungsgraden gefunden, wobei die Zunahme mit der Erhitzung überproportional ist. Die Beträge der Zugamplituden sind bei weitem ausreichend, um Kavitation zu induzieren.
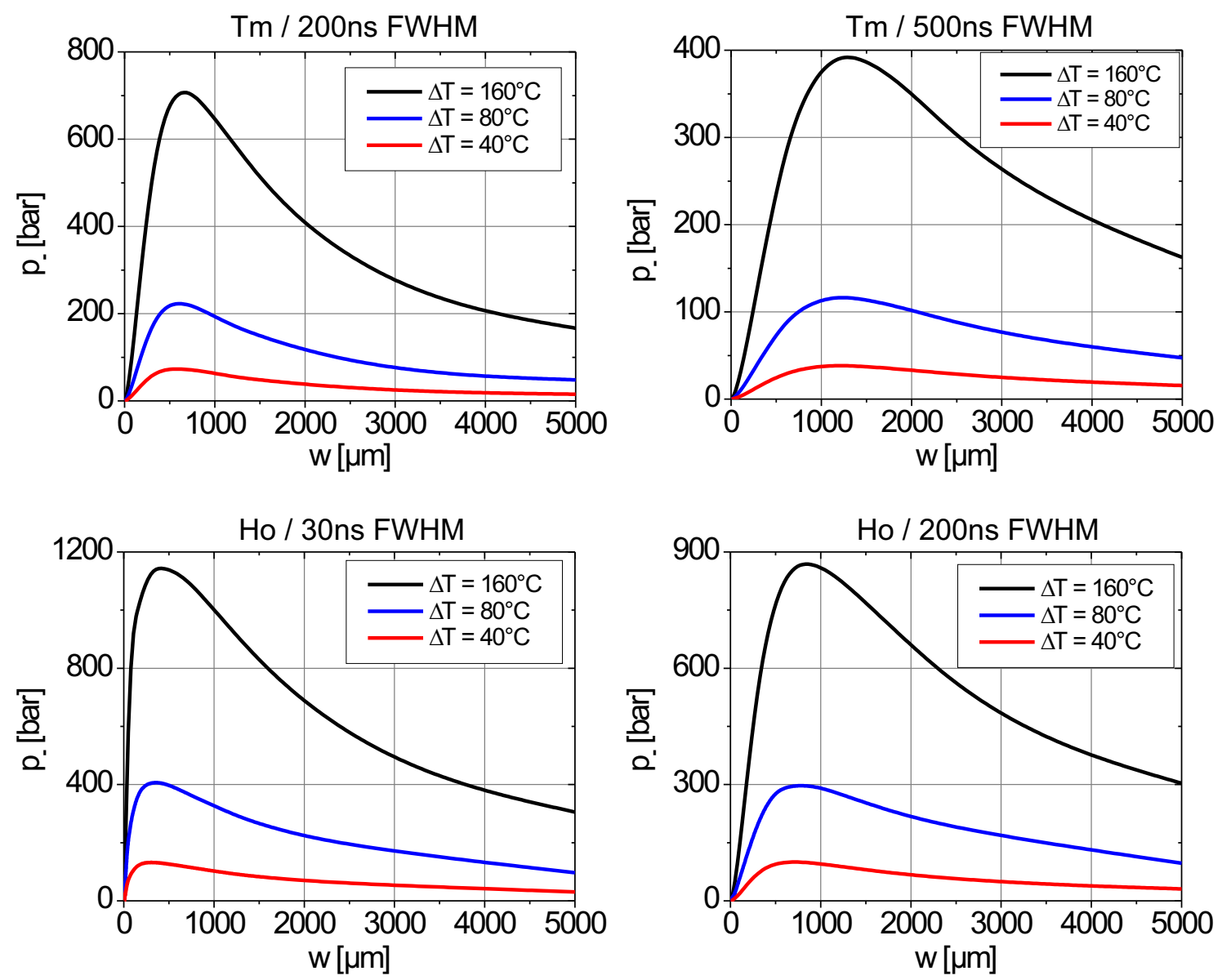

Abb. 4.10: Verlauf der Funktion $\left.p_{-}(w)\right|_{\bar{\mu}, t_{\mathrm{p}}, \Delta T}$ zur Tm- und Ho-Laseranregung mit den angegebenen Pulsdauern. Man beachte die unterschiedliche Skalierung der Ordinaten. Den Berechnungen liegen analog Abbildung 4.9 die Absorptionen $\mu_{\mathrm{Tm}}=5.94 / \mathrm{mm}\left(40^{\circ} \mathrm{C}\right)$, $5.43 / \mathrm{mm}\left(80^{\circ} \mathrm{C}\right), 4.39 / \mathrm{mm}\left(160^{\circ} \mathrm{C}\right)$, sowie $\mu_{\mathrm{Ho}}=2.97 / \mathrm{mm}\left(40^{\circ} \mathrm{C}\right), 2.77 / \mathrm{mm}$ $\left(80^{\circ} \mathrm{C}\right), 2.35 / \mathrm{mm}\left(160^{\circ} \mathrm{C}\right)$ zugrunde. 


\section{Diskussion der Verlaufsmerkmale}

Da der Zuganteil durch akustische Beugung aus dem initialen Druckprofil entsteht, hängt seine Höhe von der Höhe der Druckamplitude, aber auch von der jeweiligen Bestrahlungsgeometrie ab. Den Grenzfall stellt die eindimensionale, ebene Welle dar, in der gar keine Zuganteile auftreten (Paragrah 3.3.1). Ausgehend von $w \simeq 0$ wächst die Zugamplitude in den Verläufen $\left.p_{-}(w)\right|_{\bar{\mu}, t_{\mathrm{p}}, \Delta T}$ zunächst monoton mit der Druckamplitude an. Zu größeren Strahlradien setzt sich die geometrische Annäherung an die ebene Welle gegenüber dem asymptotisch begrenzten Druckwachstum durch und führt zu einer Abnahme der Zugamplitude. Im Wechselpunkt dieser gegenläufigen Effekte befindet sich das lokale Maximum.

\section{Nulldurchgang}

Die Verläufe zum Quotienten $\left.\left(t_{\mathrm{N}} / t_{\mathrm{p}}\right)(w)\right|_{\bar{\mu}, t_{\mathrm{p}}, \Delta T}$ sind in Abbildung 4.11 zusammengefaßt. Zur Tm-Anregung der Dauer $t_{\mathrm{p}}=500 \mathrm{~ns}$ weist der Verlauf am Kurvenbeginn $(w \approx 80 \mu \mathrm{m})$ ein lokales Minimum auf. Dieses Minimum rückt zu kürzeren Pulsdauern so nah an den Ursprung, daß es in den übrigen Verläufen zwar vorhanden, aber kaum zu erkennen ist. Mit zunehmendem Strahlradius wächst der Quotient in allen Fällen streng monoton und unbeschränkt an. Die höchsten Beträge werden bei kurzen Pulsdauern und niedrigen Absorptionen gefunden. Eine geringere Betragszunahme ist mit Zunahme des Erhitzungsgrades zu beobachten.

Ein zentrales Merkmal aller Verläufe ist deren Beschränkung auf den positiven Wertebereich. Gemäß Tabelle 4.1 gilt für positive Quotientenwerte, daß zum Zeitpunkt des Nulldurchgangs bereits mehr als 50\% der Pulsenergie in der Wasserprobe deponiert sind. $\mathrm{Ab}$ Quotientenwerten größer Eins ist praktisch die gesamte Energie deponiert (Anteil $>99 \%$ ). Da die Probenanregung bis zum Erreichen des Nulldurchgangs unter erhöhtem Druck stattfindet, begünstigen die höheren Quotientenwerte das verdampfungsfreie Erreichen der Maximaltemperatur zur jeweiligen Pulsenergie. 

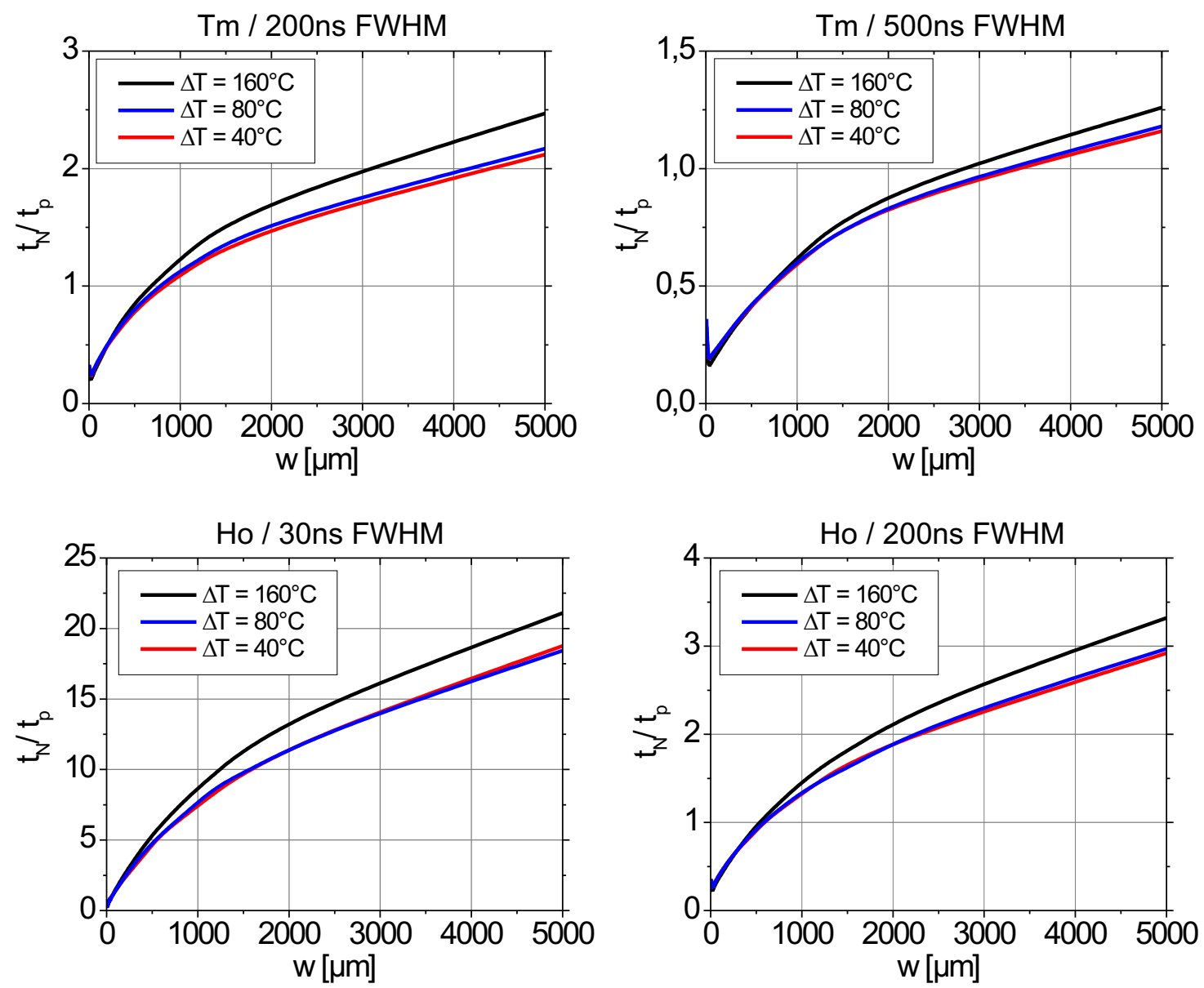

Abb. 4.11: Verlauf des Quotienten $\left.\left(t_{\mathrm{N}} / t_{\mathrm{p}}\right)(w)\right|_{\mu_{,}, t_{\mathrm{p}}, \Delta T}$ zur Tm- und Ho-Laseranregung mit den angegebenen Pulsdauern. Man beachte die unterschiedliche Skalierung der Ordinaten. Den Berechnungen liegen analog Abbildung 4.9 die Absorptionen $\mu_{\mathrm{Tm}}=5.94 / \mathrm{mm}\left(40^{\circ} \mathrm{C}\right)$, $5.43 / \mathrm{mm}\left(80^{\circ} \mathrm{C}\right), 4.39 / \mathrm{mm}\left(160^{\circ} \mathrm{C}\right)$, sowie $\mu_{\text {но }}=2.97 / \mathrm{mm}\left(40^{\circ} \mathrm{C}\right), 2.77 / \mathrm{mm}$ $\left(80^{\circ} \mathrm{C}\right), 2.35 / \mathrm{mm}\left(160^{\circ} \mathrm{C}\right)$ zugrunde. 


\section{Diskussion der Verlaufsmerkmale}

Im Unterschied zu den vorangegangenen Fällen lassen sich die Verlaufsmerkmale des Quotienten $\left(t_{\mathrm{N}} / t_{\mathrm{p}}\right)$ nicht aus den akustischen Einschlußbedingungen herleiten. Der Nulldurchgang resultiert vielmehr aus dem speziellen Verhalten der zugrunde liegenden Lösungsformeln $p_{\delta, n}(\vec{o}, t)$ (3.58) und ist daher nur durch nähere Betrachtung dieser Formeln zu deuten. Für die Beschränkung auf den positiven Wertebereich führt dies zu folgenden Überlegungen: Positive Werte des Quotienten besagen, daß der Nulldurchgang frühestens zum Zeitpunkt $t=0$ einsetzt. Das Faltungsintegral zur $n$-ten Lösungsformel hat bei $t=0$ die Darstellung

$$
\left[p_{\delta, n}(\vec{o}, \tau) \otimes g(\tau)\right](0)=\int_{0}^{\infty} p_{\delta, n}(\vec{o}, \tau) g(\tau) \mathrm{d} \tau .
$$

Um festzustellen, welches Vorzeichen dieses Integral annehmen kann, wird es am Nulldurchgang $t_{0, \delta}$ der Impulsantwort in die beiden Teilintegrale

$$
\int_{0}^{t_{0, \delta}} p_{\delta, n}(\vec{o}, \tau) g(\tau) \mathrm{d} \tau>0 \quad \text { und } \quad \int_{t_{0, \delta}}^{\infty} p_{\delta, n}(\vec{o}, \tau) g(\tau) \mathrm{d} \tau<0
$$

aufgeteilt. Weiter wird genutzt, daß $g(\tau)$ ab $\tau>0$ monoton fallend ist. Hierdurch können die beiden Integrale über den Funktionswert $g\left(t_{0, \delta}\right)$ nach unten abgeschätzt werden:

$$
\begin{gathered}
\int_{0}^{t_{0, \delta}} p_{\delta, n}(\vec{o}, \tau) g(\tau) \mathrm{d} \tau>g\left(t_{0, \delta}\right) \int_{0}^{t_{0, \delta}} p_{\delta, n}(\vec{o}, \tau) \mathrm{d} \tau \quad \text { und } \\
\int_{t_{0, \delta}}^{\infty} p_{\delta, n}(\vec{o}, \tau) g(\tau) \mathrm{d} \tau>g\left(t_{0, \delta}\right) \int_{t_{0, \delta}}^{\infty} p_{\delta, n}(\vec{o}, \tau) \mathrm{d} \tau .
\end{gathered}
$$

Damit ist gleichzeitig für das Ausgangsintegral (als Summe der beiden Teilintegrale) eine untere Abschätzung gefunden worden:

$$
\int_{0}^{\infty} p_{\delta, n}(\vec{o}, \tau) g(\tau) \mathrm{d} \tau>g\left(t_{0, \delta}\right) \int_{0}^{\infty} p_{\delta, n}(\vec{o}, \tau) \mathrm{d} \tau .
$$

Der Nutzen dieser Abschätzung liegt im Wissen um den Betrag des Integrals über $p_{\delta, n}(\vec{o}, t)$, denn gemäß Gl. (3.18) ist

$$
\int_{0}^{\infty} p_{\delta, n}(\vec{o}, \tau) \mathrm{d} \tau=0
$$


Der Funktionswert zur $p_{n}(\vec{o}, 0) n$-ten Einzellösung ist folglich immer positiv und so auch der Funktionswert

$$
p(\vec{o}, 0)=\sum_{n=1}^{n_{\max }} p_{n}(\vec{o}, 0)
$$

der Gesamtlösung (3.37). Damit ist gezeigt, daß sich die bipolare Lösungsfunktion zum Zeitpunkt $t=0$ noch in der Druckphase befindet und der Nulldurchgang nur zu einem späteren, positiven Zeitpunkt stattfinden kann.

Das unbeschränkte Wachstum von $\left(t_{\mathrm{N}} / t_{\mathrm{p}}\right)(w)$ geht auf ein vergleichbares Verhalten der Impulsantworten $p_{\delta, n}(\vec{o}, t)$ und ihres Nulldurchgangs $t_{0, \delta}$ zurück. Nach Gl. (3.16) bestimmt sich die Impulsantwort

$$
p_{\delta, n}(\vec{o}, t) \propto \frac{\partial}{\partial t}\left[\left.\frac{1}{t} \int f_{n}(\vec{r})\right|_{r=c_{0} t} \mathrm{~d}^{2} r\right]
$$

aus der sphärischen Integration über die initiale Druckerhöhung $f_{n}(\vec{r})$. Darin treten die Zuganteile auf, wenn der Betrag des Integrals abnehmend mit $t$ ist und so die zeitliche Ableitung negativ wird. Da die Integrationssphäre über den Radius $r=c_{0} t$ an die Zeit gekoppelt ist, findet diese Abnahme umso später statt, je weiter sich die initiale Druckerhöhung im Raum ausdehnt. Die Vergrößerung des Strahlradius bewirkt hierdurch eine entsprechende Verschiebung des Nulldurchgangs zu späteren Zeiten. Dies begründet auch die Zunahme von $\left(t_{\mathrm{N}} / t_{\mathrm{p}}\right)(w)$ mit abnehmender Absorption. Die Zunahme mit abnehmender Pulsdauer resultiert hingegen aus der Division durch $t_{\mathrm{p}}$.

\section{Einfluß der thermischen Nichtlinearitäten}

In den dargestellten Verlaufsmerkmalen der Druck- und Zugamplitude wurde ein mit der Erhitzung überproportionaler Amplitudenanstieg festgestellt, der auf den Einfluß thermischer Nichlinearitäten hinweist. Wie in Abschnitt 3.2 ausgeführt, kommt in der thermischen Nichtlinearität die Temperaturabhängigkeit des Grüneisenkoeffizienten zum Ausdruck. Im thermisch linearen Fall zu kleinen Bestrahlungen und nahezu konstantem Grüneisenkoeffizienten $\Gamma \simeq \Gamma_{0}$ entwickeln sich die Amplituden aus der Lösungsfunktion (3.58),

$$
\begin{aligned}
\left.p_{\delta, n}(\vec{o}, t)\right|_{n=1} & =\left[\frac{\left(1+\left(R_{\mathrm{QW}}\right)^{n}\right) \bar{\mu} n \Gamma_{n-1}\left(H_{0}\right)^{n}}{2} \cdot \hat{p}_{\delta, n}(\vec{o}, t)\right]_{n=1} \\
& =\frac{\left(1+R_{\mathrm{QW}}\right) \bar{\mu} \Gamma_{0} H_{0}}{2} \cdot \hat{p}_{\delta, 1}(\vec{o}, t)
\end{aligned}
$$


zum ersten photoakustischen Quelltermglied. Aufgrund der verbleibenden Bestrahlungsabhängigkeit der Absorption wächst die Amplitude nicht direkt proportional mit der Bestrahlung. Der monotone Abfall der Funktion $\bar{\mu}(H)$ wirkt sich dabei verschieden auf die Lösung aus: Im Vorfaktor führt er zu einem verringerten Amplitudenzuwachs mit der Bestrahlung, im normierten Lösungsanteil $\hat{p}_{\delta, 1}(\vec{o}, t)$ verstärkt er den Amplitudenzuwachs, weil geringere Absorptionen den akustischen Einschluß erhöhen. Die resultierende Entwicklung der Amplitudenfunktionen $p_{ \pm}(H, \bar{\mu}(H))$ ist in Abbildung 4.12 dargestellt und zeigt - beispielhaft für die Parameter der Tm-Laseranregung - einen quasi-linearen Verlauf. Vergleichend dazu ist die Entwicklung aufgetragen, wenn die thermische Nichtlinearität durch Hinzunahme aller Lösungsterme $p_{\delta, n}(\vec{o}, t)$ berücksichtigt wird. Das zugehörige Wachstum ist deutlich steiler und führt zu Betragsunterschieden von bis zu einem Faktor vier gegenüber dem thermisch linearen Fall. Die gewählte Bestrahlungsobergrenze entspricht der oberen Gültigkeitsgrenze der in den Rechnungen verwendeten Temperaturfunktion $\left.T(H)\right|_{\mu}$.
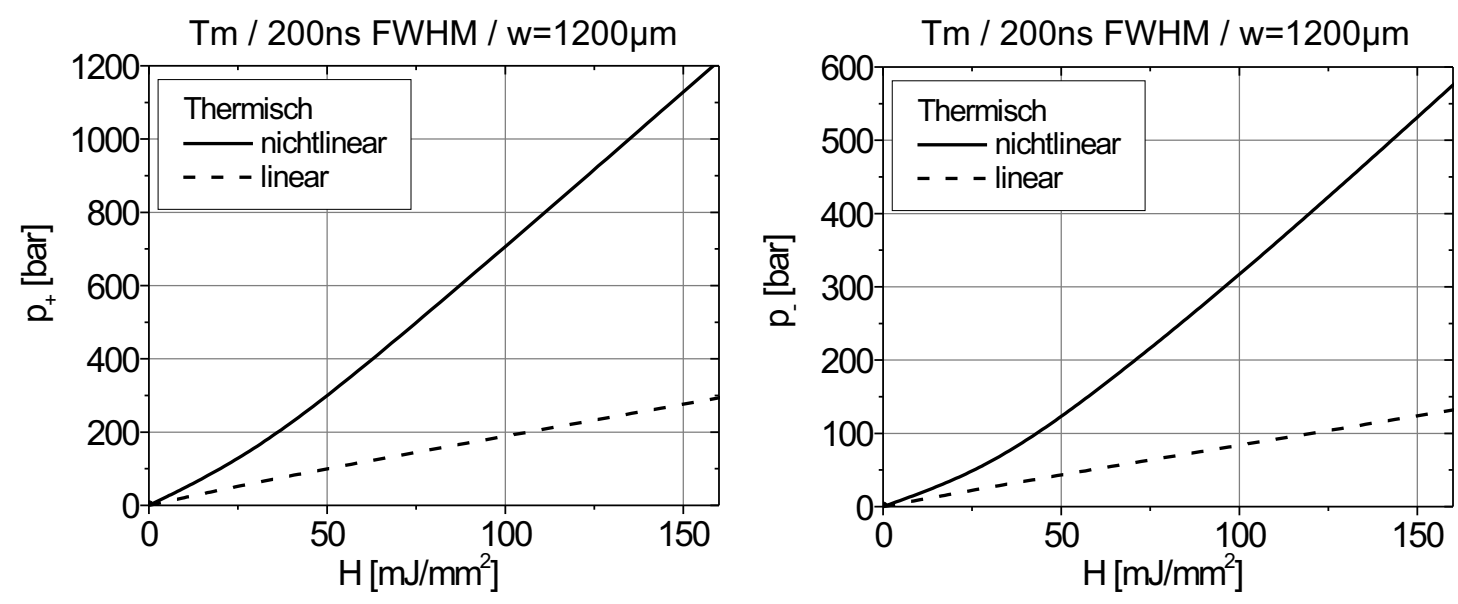

Abb. 4.12: Verlauf der Funktionen $\left.p_{ \pm}(H, \bar{\mu}(H))\right|_{w, t_{\mathrm{p}}}$ zu einer exemplarischen Tm-Laseranregung, im thermisch linearen (gestrichelte Kurve) und nichtlinearen Fall (durchgezogene Kurve). Die Temperaturerhöhungen $\Delta T=40,80,160^{\circ} \mathrm{C}$ zu den Verlaufsgraphen 4.9 und 4.10 werden bei den Bestrahlungen $H_{0}=28,62,164 \mathrm{~mJ} / \mathrm{mm}^{2}$ erreicht.

Anders als für die Amplituden wurden für den Nulldurchgangsquotienten $t_{\mathrm{N}} / t_{\mathrm{p}}$ nur geringe Änderungen mit der Bestrahlung festgestellt (Abbildung 4.11). Die zeitliche Lage des Nulldurchgangs $t_{\mathrm{N}}$ bestimmt sich aus den Verlaufsformen der normierten Lösungsanteile $\hat{p}_{\delta, n}(\vec{o}, t)$. Darin ändern sich mit der Bestrahlung allein die Absorptionswerte. In die Gesamtlösung fließt die Bestrahlung noch als Gewichtungsfaktor $\propto H_{0}^{n}$ für die superponierten Einzellösungen ein. Bei Betrachtung des vollständigen Lösungsverlaufs $p(\vec{o}, t) \mathrm{zu}$ 
verschiedenen Bestrahlungen zeigt sich, daß sich die Verlaufsform insgesamt nur wenig ändert (Abbildung 4.13). Der Einfluß der thermischen Nichtlinearität ist demnach bedeutender für die Höhe der erreichten Amplituden.

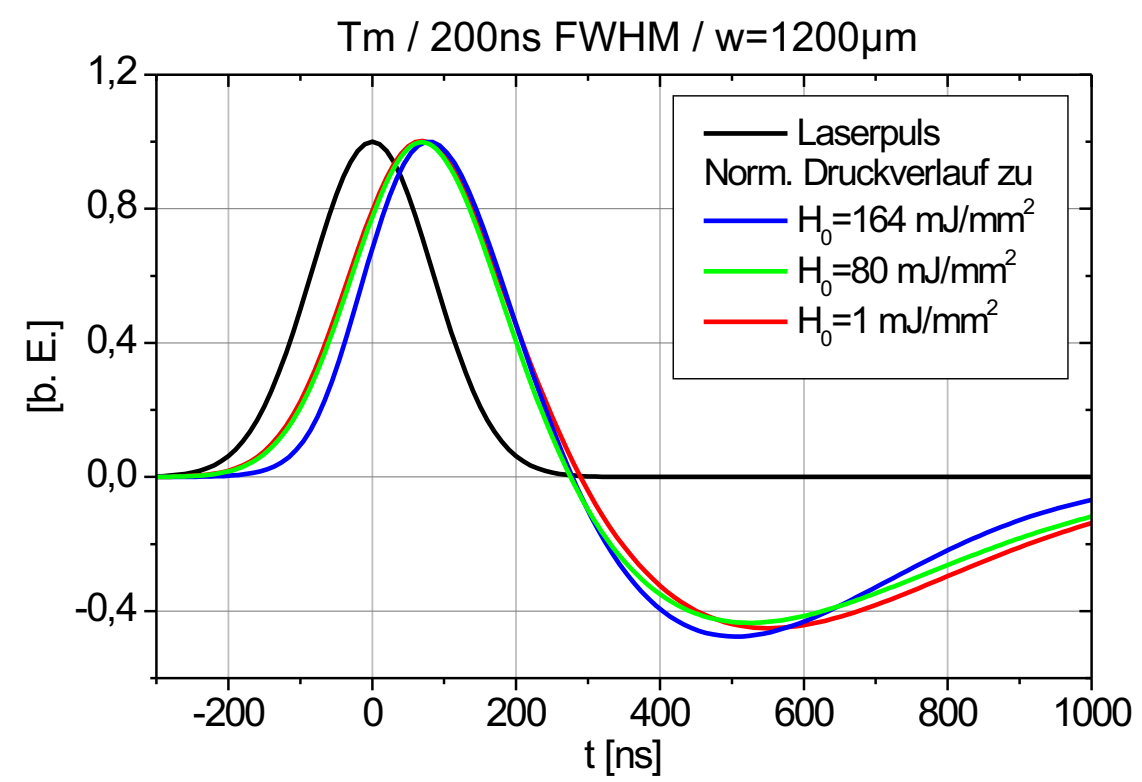

Abb. 4.13: Normierte Druckverläufe $\left.\hat{p}(\vec{o}, t)\right|_{\bar{\mu}\left(H_{0}\right)}$ zu den Parametern einer exemplarischen TmLaseranregung mit verschiedenen Bestrahlungen $H_{0}$. Bezugspunkt der Normierung ist das Maximum der Kurven.

\subsubsection{Betrachtung bei konstanter Pulsenergie}

\section{Druck- und Zugamplitude}

Für konstante Pulsenergie berechnen sich die Druck- und Zugamplitude zu jedem Strahlradius $w$ aus einer individuellen Bestrahlung $H_{0}=2 E /\left(\pi w^{2}\right)$ und Absorption $\bar{\mu}(H)$. Die Verläufe $\left.p_{ \pm}(w)\right|_{t_{\mathrm{p}}, E}$ sind in den Abbildungen 4.14 und 4.15 zusammengestellt. Die Verläufe sind einheitlich durch einen streng monotonen Abfall gekennzeichnet. Aufgrund der Schnelligkeit des Abfalls sind die Kurven nur bis zu einem Strahlradius von $3000 \mu \mathrm{m}$ berechnet, gegenüber $5000 \mu \mathrm{m}$ in den Abbildungen 4.9 bis 4.11 . $\mathrm{Zu}$ kleinen Strahlradien sind die Verläufe durch den Gültigkeitsbereich der zugrunde gelegten Temperaturfunktionen $\left.T(H)\right|_{\mu}$ begrenzt. Die zugehörigen Bestrahlungsgrenzen liegen bei $H_{0}=170 \mathrm{~mJ} / \mathrm{mm}^{2}$ (Tm-Laser) und $H_{0}=300 \mathrm{~mJ} / \mathrm{mm}^{2}$ (Ho-Laser). Die höchsten Amplituden werden bei hohen Pulsenergien und kurzen Pulsdauern erreicht. 

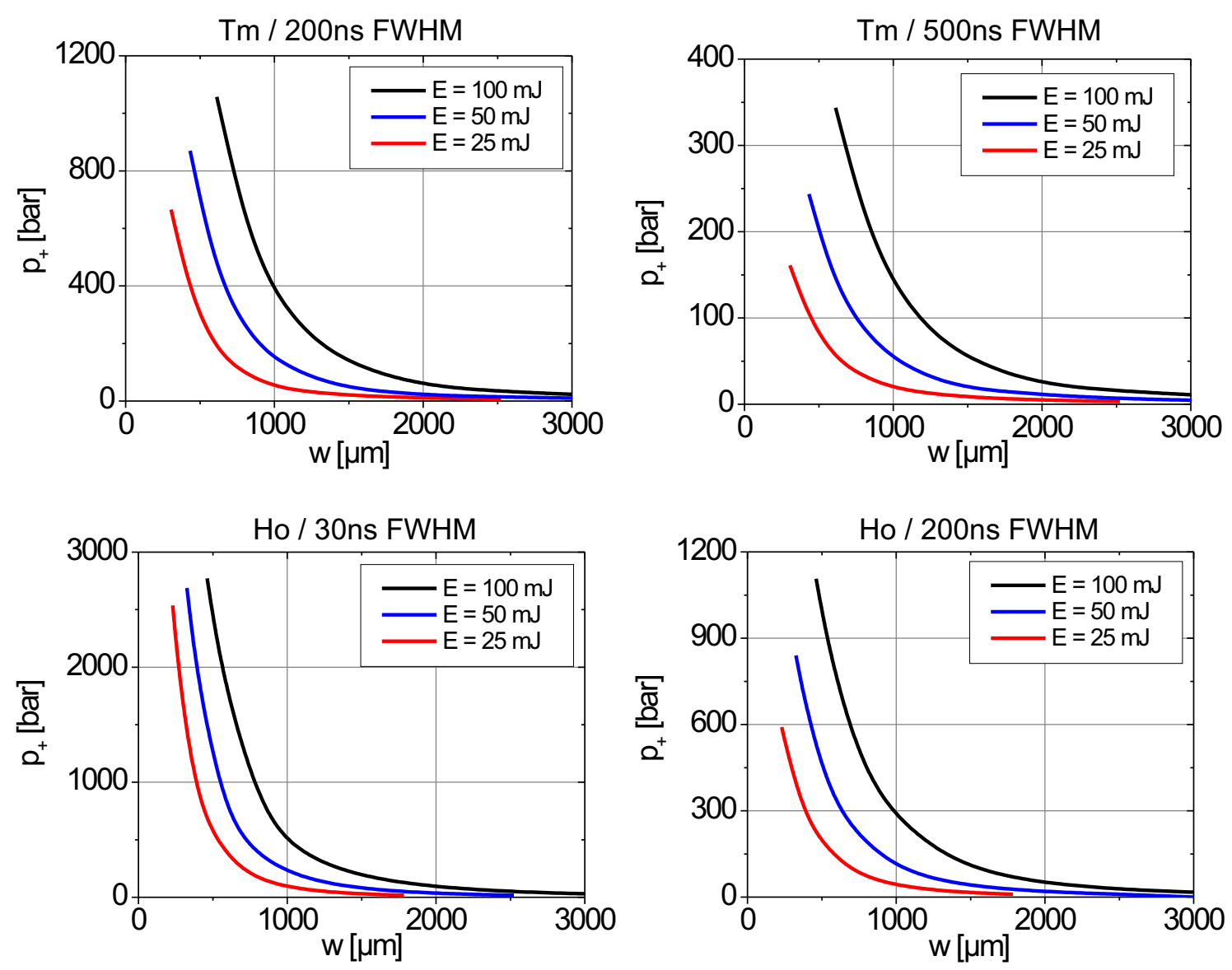

Abb. 4.14: Verlauf der Funktion $\left.p_{+}(w)\right|_{t_{\mathrm{p}}, E}$ zur Tm- und Ho-Laseranregung mit den angegebenen Pulsdauern. Man beachte die unterschiedliche Skalierung der Ordinaten.

\section{Diskussion}

Der einheitlich reziproke Verlaufscharakter der Funktionen $\left.p_{ \pm}(w)\right|_{t_{\mathrm{p}}, E}$ legt nahe, daß die Änderungen der Bestrahlung $\propto 1 / w^{2}$ die Verlaufsformen dominieren. Die Änderungen der Bestrahlung gehen zur Potenz $n$ in die Lösungsfunktionen

$$
p_{\delta, n}(\vec{o}, t)=\frac{\left(1+\left(R_{\mathrm{QW}}\right)^{n}\right) \bar{\mu} n \Gamma_{n-1}\left(H_{0}\right)^{n}}{2} \cdot \hat{p}_{\delta, n}(\vec{o}, t)
$$

ein und überlagern sich dort mit den Änderungen von Absorption und normiertem Lösungsanteil $\hat{p}_{\delta, n}(\vec{o}, t)$. Die Amplitudenentwicklung aus dem normierten Lösungsanteil ist durch die Verläufe zur Annahme eines konstanten Erhitzungsgrades beschrieben. Für große Strahlradien wirkt die Funktion $\hat{p}_{\delta, n}(\vec{o}, t)$ der Bestrahlungsabnahme $\propto 1 / w^{2 n}$ nicht entgegen: Der zugehörige Verlauf $\left.p_{+}(w)\right|_{\bar{\mu}, t_{\mathrm{p}}, \Delta T}$ läuft für alle $\bar{\mu}$ gegen einen asymptotischen Grenzwert (Abbildung 4.9) und die Funktion $\left.p_{-}(w)\right|_{\bar{\mu}, t_{\mathrm{p}}, \Delta T}$ geht selbst gegen Null 

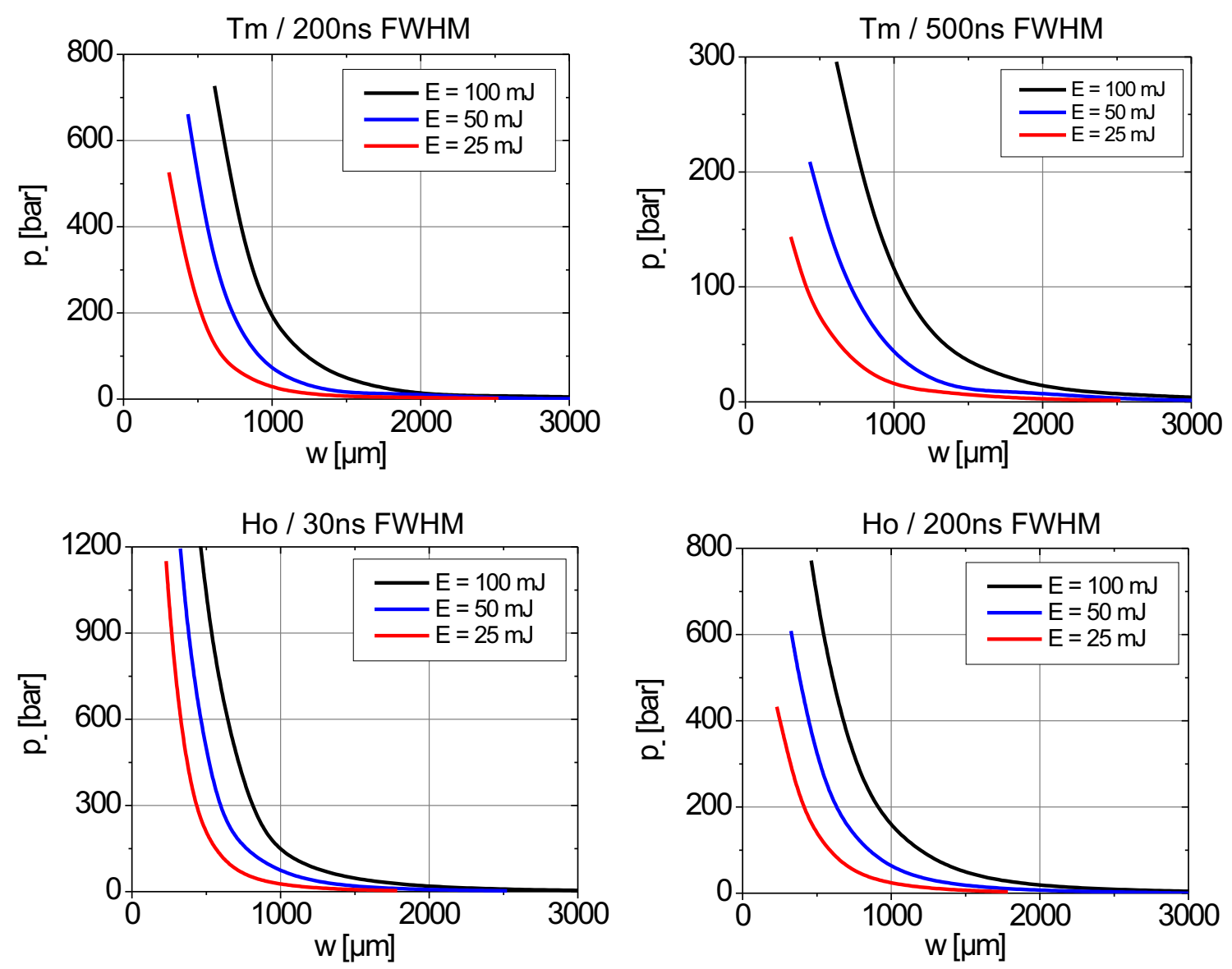

Abb. 4.15: Verlauf der Funktion $\left.p_{-}(w)\right|_{t_{\mathrm{p}}, E}$ zur Tm- und Ho-Laseranregung mit den angegebenen Pulsdauern. Man beachte die unterschiedliche Skalierung der Ordinaten.

(Abbildung 4.10). Umgekehrt ist der Bestrahlungszuwachs in Richtung kleiner Strahlradien so ausgeprägt, daß der allmähliche Amplitudenrückgang in $\hat{p}_{\delta, n}(\vec{o}, t)$ das Amplitudenwachstum nicht kompensieren kann (im Bereich der betrachteten Strahlradien). Im Fall der Funktion $\left.p_{-}(w)\right|_{\bar{\mu}, t_{\mathrm{p}}, \Delta T}$ muß zudem erst das lokale Maximum überschritten sein, bis die Amplitude in Richtung kleiner Strahlradien abnimmt. Die Auswirkungen der Absorptionsänderungen wurden im vorigen Unterabschnitt beschrieben: Im Vorfaktor führt der monotone Abfall der Funktion $\bar{\mu}(H)$ zu einer direkten Amplitudenabnahme, innerhalb von $\hat{p}_{\delta, n}(\vec{o}, t)$ bewirkt er eine Zunahme, bedingt durch die Verbesserung der akustischen Einschlußbedingungen. 


\section{Nulldurchgang}

Die Verläufe zum Nulldurchgangsquotienten $\left.\left(t_{\mathrm{N}} / t_{\mathrm{p}}\right)(w)\right|_{E}$ sind in Abbildung 4.16 zusammengefaßt. Die Berechnungen erfolgten über dieselben Strahlradienintervalle wie zur Druck- und Zugamplitude. Alle Quotienten sind positiv und wachsen mit zunehmendem Strahlradius monoton an. Die höchsten Beträge werden zu kurzen Pulsdauern und niedrigen Absorptionen gefunden. Zwischen den Verläufen bei unterschiedlicher Pulsenergie sind nur geringe Unterschiede zu beobachten.
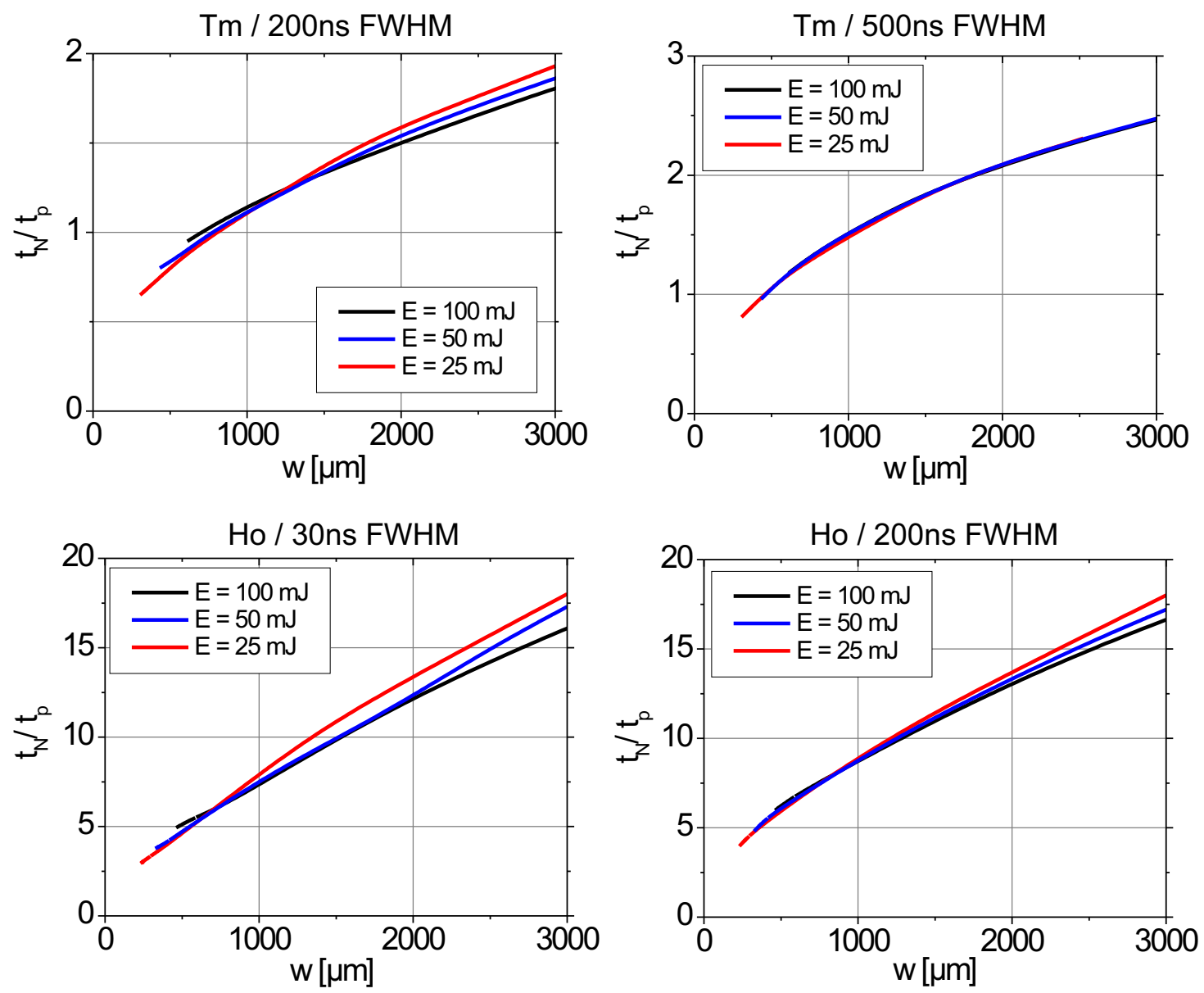

Abb. 4.16: Verlauf des Quotienten $\left.\left(t_{\mathrm{N}} / t_{\mathrm{p}}\right)(w)\right|_{E}$ zur Tm- und Ho-Laseranregung mit den angegebenen Pulsdauern. Man beachte die unterschiedliche Skalierung der Ordinaten. 


\section{Diskussion}

Wie im vorigen Unterabschnitt dargestellt, ändert sich die zeitliche Lage des Nulldurchgangs nur wenig mit der Bestrahlung. Den Haupteinfluß üben die Änderungen der Bestrahlungsgeometrie mit dem Strahlradius aus. Die Kurven zu unterschiedlichen Pulsenergien liegen daher dicht beieinander und ähneln in Betrag und Verlauf den Kurven zur Annahme eines konstanten Erhitzungsgrades.

\subsection{Fasergeführte Laseranregung}

In Abschnitt 2.1 wurde erörtert, daß das Strahlprofil fasergeführter Laser statistisch moduliert ist. Die Bestrahlungsmodulationen bilden sich im Erhitzungsprofil der Wasserprobe direkt ab [24, 32], da der wärmeleitungsbedingte Temperaturausgleich über Mikrosekunden und damit langsam in bezug auf die betrachteten Anregungsdauern stattfindet. Da die Höhe der Modulationen je nach experimentellen Bedingungen stark variieren kann (Kohärenzlänge des Lasers, Fasergeometrie usw.), ist eine Vorhersage des tatsächlichen Erhitzungsgrades in der Wasserprobe sehr schwierig. Als Interferenzerscheinung liegen die Abmessungen der Modulationen im Bereich der Laserwellenlänge. Für die einzelne Überhöhung ist der akustische Einschluß daher nur gering, so daß sich die Modulationen nur abgeschwächt im Schallfeld abbilden. Die Amplitudenverhältnisse zwischen der makroskopischen Schallentwicklung aus der Fasergeometrie und den lokalen Bestrahlungsüberhöhungen seien an folgendem Zahlenbeispiel illustriert: Am Austritt einer Multimode-Faser vom Radius $a=315 \mu \mathrm{m}$, die gütegeschaltete Ho-Laserpulse der Dauer $t_{\mathrm{p}}=30 \mathrm{~ns}$ führt, entstehen zu einer Bestrahlung $H=75 \mathrm{~mJ} / \mathrm{mm}^{2}$ Druck- und Zugamplituden von max. +430 und -790 bar (im Faserfall übersteigen die Zugamplituden die initialen Druckamplituden, siehe unten). Die lokale Modulation sei als gaußförmig mit Radius $w=2 \mu \mathrm{m}$ angenommen und zeichne sich durch eine 4-fach höhere Bestrahlung gegenüber dem Mittel aus [24]. Aus der resultierenden Bestrahlung von $300 \mathrm{~mJ} / \mathrm{mm}^{2}$ entwickeln sich Druck- und Zugamplituden von max. +8 und -6 bar. Dies sind weniger als 2\% der Amplitudenbeträge des Gesamtfeldes.

Die Gesamtentwicklung des Schallfeldes läßt sich beispielhaft am Verlauf in der Eintrittsebene $z=0$ darstellen. Vergleichend zur Gaußschen Laseranregung (Abschnitt 4.1) sei der Verlauf zu einem Faserradius $a=315 \mu \mathrm{m}$ und der Ruheabsorption $\mu\left(20^{\circ} \mathrm{C}\right)=$ 6.48/mm der Tm-Laserwellenlänge betrachet. Desgleichen sei für eine erste Übersicht 
der Fall einer Anregung mit Bestrahlung $H \rightarrow 0$ angenommen, so daß sich das Schallfeld allein aus der Impulsantwort $p_{\delta, 1}\left(q_{x}, t\right)$ (3.69) zum linearen Quelltermanteil entwickelt. Der Verlauf der Impulsantwort ist in Abbildung 4.17 zu sehen. Darin ist in den ersten vier Graphen das Top-Hat-Strahlprofil des anregenden Lasers (rote Kurven) aufgetragen. Unmittelbar nach dem instantanen Energieeintrag lösen sich an den Flanken der Druckerhöhung Störungen ab, die sich in Richtung Fasermitte bewegen und den Ausgangspunkt für einen ringförmigen Zuganteil bilden $(t=90 \mathrm{~ns})$. Der Zuganteil läuft weiter zusammen und superponiert in der Fasermitte zu einem lokalen Minimum $(t=230 \mathrm{~ns})$, dessen Amplitude höher ist als die Amplitude der initialen Druckerhöhung. Bei räumlicher Betrachtung des Schallfeldes zeigt sich, daß sich das zentrale Zugminimum axial in die Tiefe fortsetzt ([6]). Frenz et al. haben an dieser Stelle die bevorzugte Bildung von Kavitationsblasen nachgewiesen [25]. Nach der Fokussierung auf die Fasermitte läuft der Zuganteil wieder ringförming auseinander und breitet sich mit den verbleibenden Druckanteilen als bipolare Welle aus.

Die Schallentwicklung verändert sich signifikant, wenn die Anregung zeitlich ausgedehnt ist. Für eine angenommene Pulsdauer $t_{\mathrm{p}}=300 \mathrm{~ns}$ ergibt sich der in Abbildung 4.18 gezeigte Verlauf: Aus dem Vergleich mit dem rot eingetragenen Laserstrahlprofil ist ersichtlich, daß sich das Schallfeld schon früh vom Top-Hat-Anregungsprofil und insbesondere von dessen steilen Flanken löst. Nach Erreichen des Druckmaximums bei $t=15 \mathrm{~ns}$ heben sich an den Flanken des Schallfeldes Störungen ab, die sich jedoch nicht zum Ausgangspunkt der Zugwellenphase entwickeln. Vielmehr fällt das Duckprofil von der Mitte her zusammen und bildet die ersten Zuganteile entsprechend in der Fasermitte aus $(t=165 \mathrm{~ns})$. An dieser Stelle ist auch das Zugminimium zu beobachten $(t=305 \mathrm{~ns})$. Ab dem Auftreten der ersten Zuganteile ist die weitere Schallentwicklung kaum von der zu einer Gaußschen Laseranregung zu unterscheiden (Abbildung 4.2). 

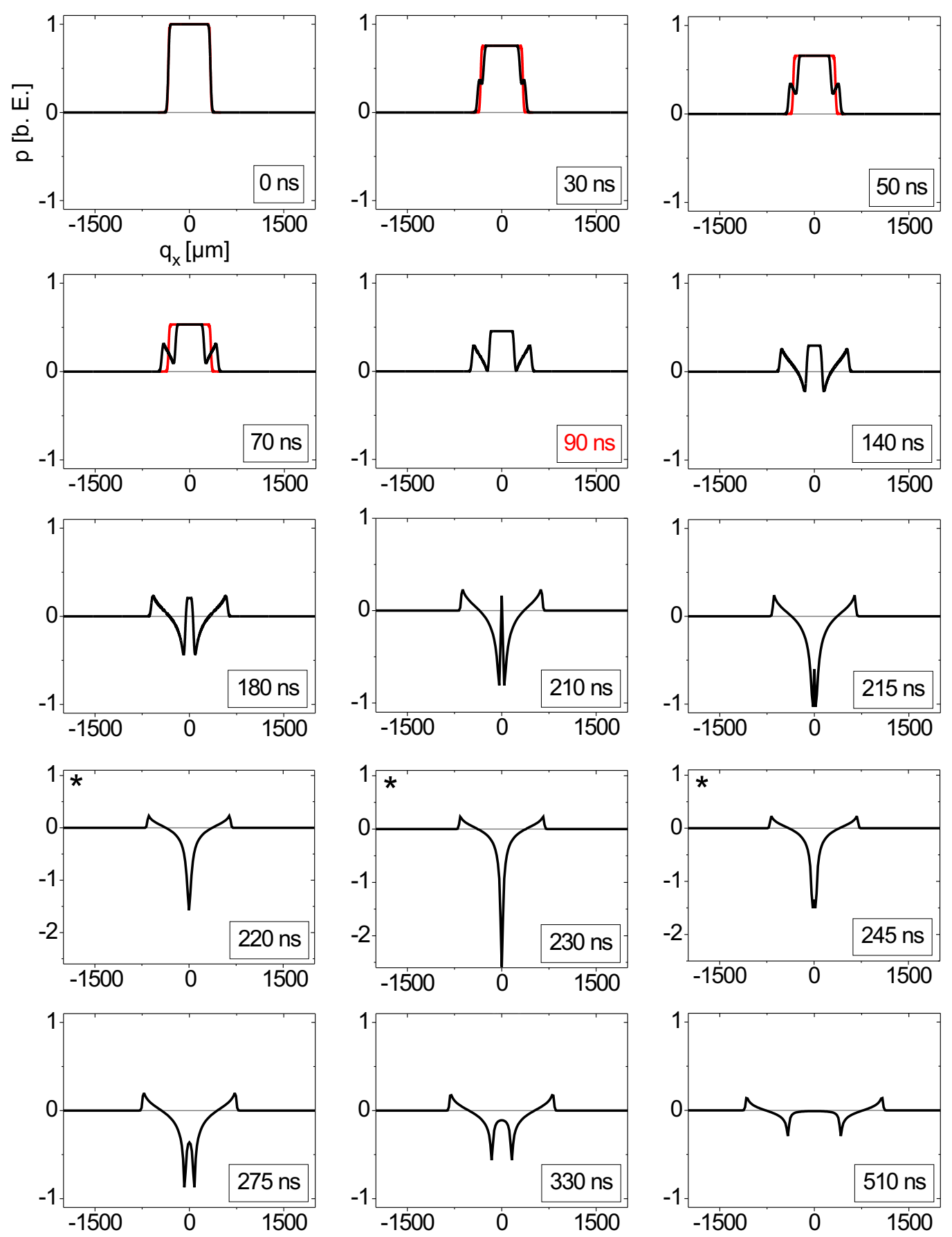

Abb. 4.17: Schallentwicklung in der Eintrittsebene (Radialschnitt) zu einer instantanen Anregung mit Faserradius $a=315 \mu \mathrm{m}$ und Absorption $\mu=6.48 / \mathrm{mm}$. Dazu in Rot das initiale Top-Hat-Anregungsprofil. Man beachte die veränderte Druck-Skalierung für die mit einem * markierten Graphen. 

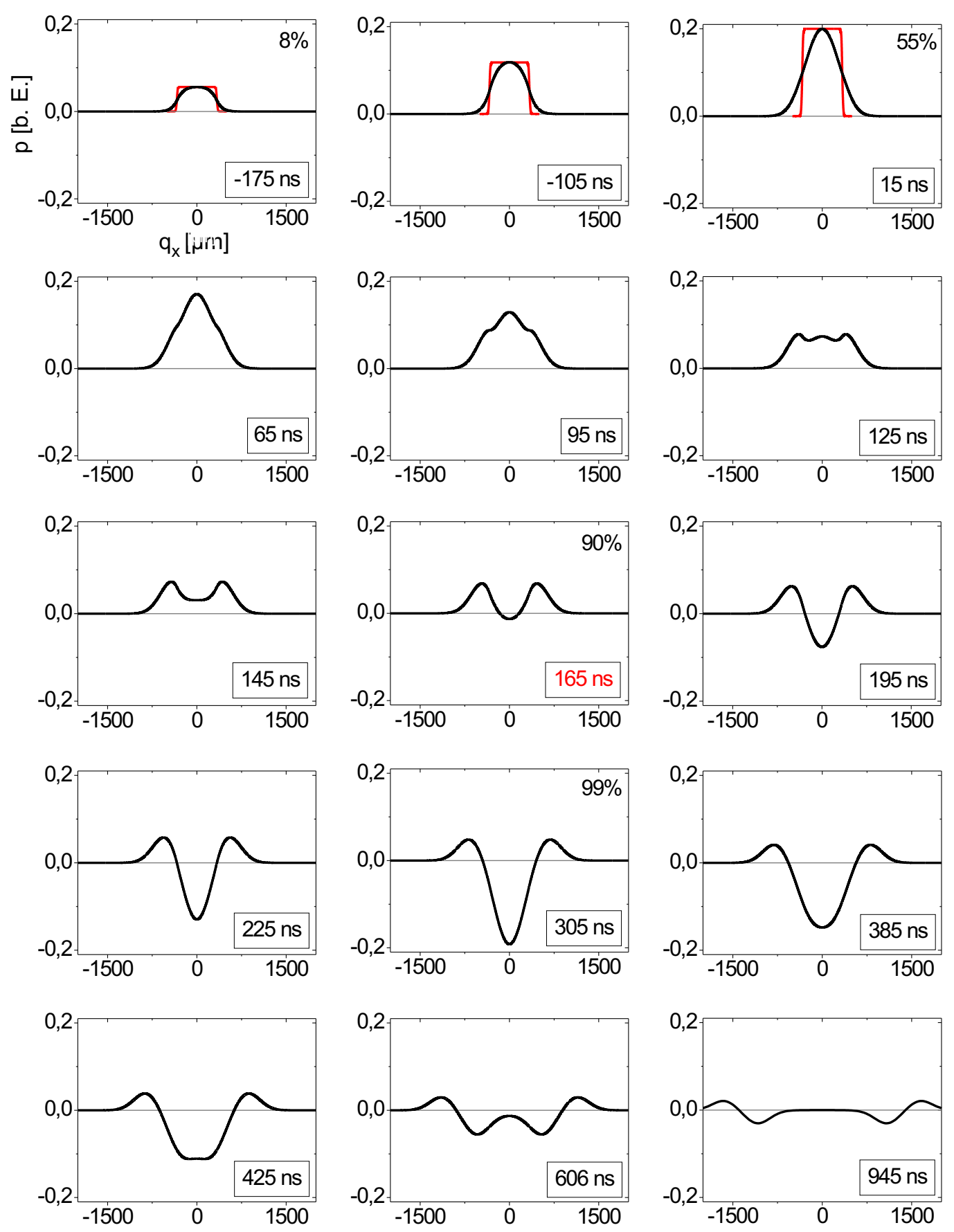

Abb. 4.18: Schallentwicklung in der Eintrittsebene (Radialschnitt) zu den Anregungsparametern $a=315 \mu \mathrm{m}, \mu=6.48 / \mathrm{mm}$ und $t_{\mathrm{p}}=300 \mathrm{~ns}$. Dazu in Rot das Top-Hat-Strahlprofil der Laseranregung. Die in einzelnen Graphen genannten Prozentzahlen entsprechen dem bis zum Graphenzeitpunkt deponierten Energieanteil. 


\section{Diskussion}

Die Impulsantwort $p_{\delta, 1}(\vec{o}, t)$ zu den dargestellten Verläufen bestimmt sich aus der sphärischen Integration (3.16)

$$
p_{\delta, 1}(\vec{o}, t) \propto \frac{\partial}{\partial t}\left[\left.\frac{1}{t} \int f_{1}(\vec{r})\right|_{r=c_{0} t} \mathrm{~d}^{2} r\right],
$$

über das initiale Top-hat-Strahlprofil $f_{1}(\vec{r})$. Mit der zeitlichen Ableitung vor dem Integral treten Zuganteile von umso größerer Amplitude auf, je schneller der Betrag des Integrals mit dem Radius $r=c_{0} t$ abnimmt. Im Fall des Top-hat-Strahlprofils geschieht dies, wenn die Integrationssphäre die steilen Flanken des Top-hat-Profils überstreicht. Die Flanken bilden hierdurch den Ausgangspunkt für die Zuganteile in der Drucktransiente [6]. Eine leichte Abschwächung erfährt dieser Effekt dadurch, daß die Betragsänderungen der wachsenden Integrationssphäre nicht allein von der radialen Strahlgeometrie, sondern auch vom Absorptionsprofil über die Tiefe $z$ bestimmt werden.

Bei der zeitlich ausgedehnten Anregung generiert jeder differentielle Bestrahlungsanteil $\mathrm{d} H=I \mathrm{~d} t$ des Laserpulses einen Schallimpuls $p_{\delta, 1}(\vec{o}, t, \mathrm{~d} H(t))$, der sich unmittelbar nach seiner Entstehung ausbreitet. Weil Schallentstehung und Schallausbreitung so über die Dauer des Laserpulses zusammenfallen, wird das Top-hat-Anregungsprofil „,abgerundet“ im Schallfeldprofil wiedergegeben. Insbesondere beginnt die Zugphase nun im Zentrum der Anregung und nicht mehr im Bereich der Flanken. Der Beginn der Zugamplitude korrespondiert hierbei mit dem Zeitpunkt $t_{\mathrm{N}}$ des Nulldurchgangs, wie er bei den charakteristischen Schallfeldgrößen beschrieben ist.

Für Anregungsdauern zwischen Null und den betrachteten 300 ns variiert die räumliche Lage des Nulldurchgangs zwischen den Flanken und der Strahlmitte (vgl. Unterabschnitt 5.1). Hierin besteht ein wesentlicher Unterschied zur Anregung mit dem Gaußschen Laserstrahl: Für die Gaußsche Bestrahlungsgeometrie findet sich, neben den Betragsmaxima von Druck und Zug, der Absolutbeginn der Zugamplitude zu jeder Pulsdauer immer in der Strahlmitte. Nur dadurch ist es möglich, Aussagen zum Zustandsverlauf der gesamten Wasserprobe durch exemplarische Betrachtungen in der Strahlmitte zu gewinnen. Im Fall der fasergeführten Anregung sind Lage und Zeitpunkt des Nulldurchgangs für jede Pulsdauer individuell zu berechnen und damit auch der Verlauf des Zustandspfades. Erschwert werden die Vorhersagen zum Zustandsverlauf durch die speckelbedingten Irregularitäten im Temperaturprofil. 


\section{Modell und Experiment im Vergleich}

Die im vorigen Kapitel errechneten Beträge für die Druckamplitude und den relativen Nulldurchgang $t_{\mathrm{N}} / t_{\mathrm{p}}$ implizieren, daß die Schallentwicklung zu typischen Tm- und HoLaseranregungen den Ablationsbeginn über weite Teile der Energiedeponierung unterdrücken kann. In der Literatur wird der Einfluß laserinduzierter Drucktransienten erstmalig von D. Albagli et al. [1] und A. Oraevsky et al. [62] in das Zustandsdiagramm der angeregten Wasser- bzw. Gewebeprobe übertragen. Motiviert sind die Überlegungen durch experimentell ermittelte Ablationsschwellen in Gewebe und wässrigen Farbstofflösungen bei Anregung durch gepulste Excimer- bzw. Nd:YAG-Laser. Da die Autoren die Druckeinwirkung dabei nur zu Temperaturen unterhalb von $100^{\circ} \mathrm{C}$ beschreiben, wird die Möglichkeit einer transienten Überhitzung nicht in Betracht gezogen. Zudem ist in beiden Arbeiten der Zustandsverlauf nur qualitativ beschrieben und nicht aus der zeitlichen Temperatur- und Druckentwicklung zusammengefügt.

R. Brinkmann et al. [10] und M. Frenz et al. [24] haben unabhängig voneinander untersucht, welche Bestrahlungsschwellen zur Wasserablation mit fasergeführten Tm- bzw. Ho-Laserpulsen erforderlich sind. Die Schwellen wurden durch Integration über das zeitliche Laserpulsprofil bis zum festgestellten Ablationsbeginn ermittelt. Die Autoren nennen hierzu auch Startzeitpunkte der Ablation, die sich auf den Beginn des Laserpulses beziehen. Da der Beginn des Laserpulses jedoch nicht näher definiert ist, sind die Ablationszeiten mit einer Ungenauigkeit von ungefähr der halben Pulsdauer (FWHM) behaftet. Für die von Frenz et. al. genannten Daten bedeutet dies eine Ungenauigkeit von $\simeq 20 \mathrm{~ns}$, bei Brinkmann et al. sind es $\simeq 200$ ns. Letztgenannte Ungenauigkeit ist zu groß, um eine weitere Auswertung der Daten vorzunehmen.

In Folgearbeiten vom Verfasser dieser Arbeit gemeinsam mit R. Brinkmann [7, 9] ist der Ablationszeitpunkt zu verschiedenen Gaußschen Tm- und Ho-Laseranregungen gezielt 
untersucht worden. Der Startzeitpunkt der Ablation war in diesen Arbeiten durch Bezug auf das Maximum des zeitlichen Laserpulsprofils eindeutig festgelegt. Die Ergebnisse dieser Experimente sowie der Experimente von M. Frenz et al. werden nachfolgend zum Vergleich mit den Modellvorhersagen dieser Arbeit herangezogen.

\subsection{Startzeitpunkte der Ablation}

Die Untersuchung des laserinduzierten Ablationsbeginns erfolgte bei Frenz et al. [24] in Proben aus destilliertem Wasser, bei Brendel und Brinkmann [9] in Proben aus entgastem Reinstwasser, um reproduzierbare Ablationsbedingungen zu gewährleisten. Der Gaußsche Laserstrahl wurde durch ein Quarzprisma hindurch in die Wasserproben geleitet, so daß die Ablation analog zur fasergeführten Laseranwendung an einer eingetauchten Quarzoberfläche stattfand (vgl. Abschnitt 2.1).

Brendel und Brinkmann untersuchten den Ablationsbeginn exemplarisch für die Anregungen mit einem gütegeschalteten Tm- und Ho-Laser $\left(t_{\mathrm{p}}=215 \mathrm{~ns}, w=328 \pm 12 \mu \mathrm{m}\right.$ bzw. $\left.t_{\mathrm{p}}=80 \mathrm{~ns}, w=205 \pm 8 \mu \mathrm{m}\right)$, sowie mit dem ersten Spike eines freilaufenden TmLasers $\left(t_{\mathrm{p}}=1.0 \mu \mathrm{s}, w=56 \pm 2 \mu \mathrm{m}\right)$. Aufgrund der längeren Pulsdauer und des niedrig gewählten Strahlradius repräsentiert der Tm-Laserspike eine Anregungsform mit geringem akustischem Einschluß. Frenz et al. verwendeten freilaufende und gütegeschaltete Ho-Laserpulse ( $t_{\mathrm{p}}=80 \mathrm{~ns}$ ), die durch eine Multimode-Quarzfaser (Radius $a=150 \mu \mathrm{m}$ ) in die Wasserproben geleitet wurden. Die Ablationsvorgänge am eingetauchten Faserende wurden von der Seite und in Aufsicht durch zwei Einzelbild-CCD-Kameras festgehalten. In analoger Weise machten Brendel und Brinkmann Einzelbildaufnahmen von den Ablationsvorgängen an der eingetauchten Prismenoberfläche. Dazu wurde mit Hilfe eines Probelasers der Reflexionsgrad an der eingetauchten Prismenoberfläche gemessen. Der Reflexionsgrad steigt sprunghaft an, sobald Dampf- oder Kaviationsblasen an der Oberfläche den Ablationsbeginn signalisieren [7]. Diese Oberflächen-Reflektometrie zeichnet sich durch eine hohe Empfindlichkeit aus und erlaubt gegenüber der Einzelbildbeobachtung eine Online-Detektion des Ablationsbeginns.

An der Schwelle zur Ablation fanden Brendel und Brinkmann eine sigmoide Verteilung der Ablationshäufigkeit über der Bestrahlung ( $N=120$ Messungen) [7, 9]. Die Verteilung charakterisieren sie über die Lage des Mittelpunktes $E D_{50}\left[\mathrm{~mJ} / \mathrm{mm}^{2}\right]$ zu einer $50 \%$-igen Ablationshäufigkeit. Anschließend erhöhten sie die Bestrahlung um definierte Vielfache des $E D_{50}$-Wertes und beobachteten die zeitlichen Änderungen des Ablationsbeginns. Als 
Startzeitpunkte definierten sie die jeweiligen Mittelwerte aus $N=25$ Messungen zu fester Bestrahlung.

Für die Anregung mit dem gütegschalteten Tm- und Ho-Laser ergaben sich die in den Abbildungen 5.1 und 5.2 aufgetragenen Zeitpunkte. Ihnen ist gemein, für alle Bestrahlungen nah am Ende der Laserpulse zu liegen. Auch bei mehr als dem dreifachen $E D_{50}$-Wert beginnt die Ablation erst, nachdem über 90\% der Pulsenergie deponiert sind. Die erreichten Endtemperaturen wachsen entsprechend an.

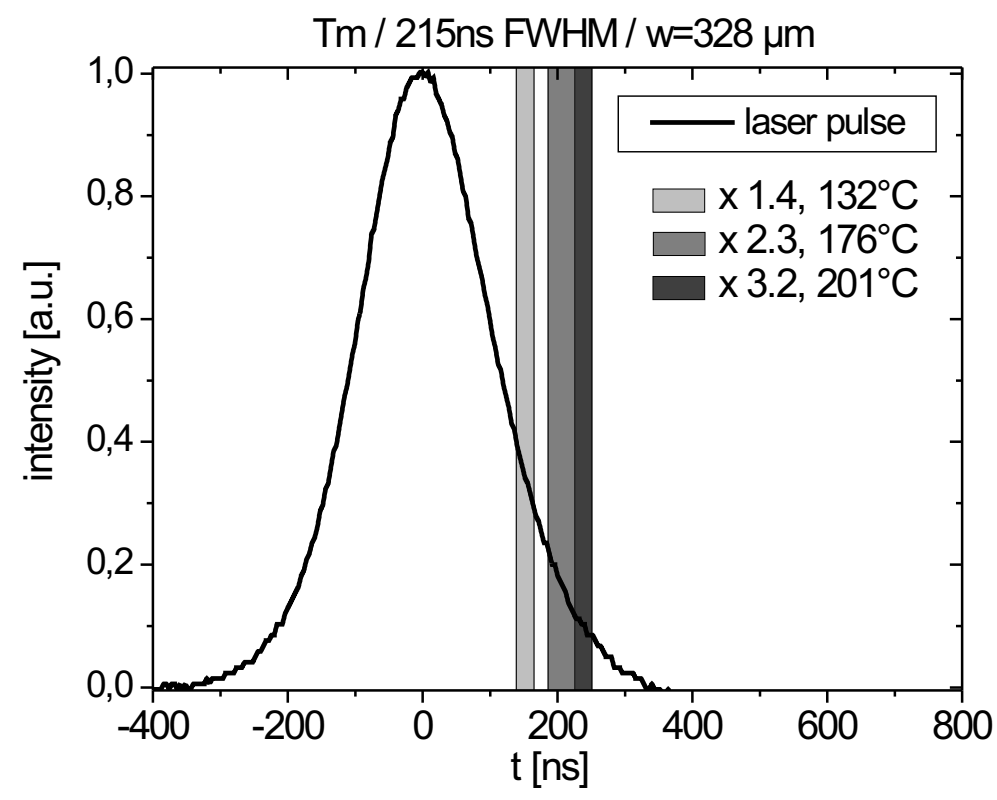

Abb. 5.1: Zeitliches Pulsprofil des gütegeschalteten Tm-Lasers und Startzeitpunkte der Ablation [9]. Die Balken markieren den mittleren Ablationsbeginn ( \pm Standardabweichung) zum angegebenen Vielfachen der Bestrahlungsschwelle $E D_{50}=68 \mathrm{~mJ} / \mathrm{mm}^{2}$. Dazu sind die Endtemperaturen angegeben, die bis zum jeweiligen Ablationsbeginn erreicht wurden. An der Schwelle ist $T\left(E D_{50}\right)=106^{\circ} \mathrm{C}$.

Bei der Anregung mit dem ersten Spike des freilaufenden Tm-Lasers (Abbildung 5.3) verschiebt sich der Ablationsbeginn merklich: zum dreifachen des $E D_{50}$-Wertes bis zur Mitte des Laserpulses und bei nochmaliger Verdopplung bis in die vordere Pulshälfte. Bedingt durch den asymptotischen Verlauf der Temperaturkurven $T(H)$ liegen die berechneten Endtemperaturen eng beineinander. Da die verwendeten Bestrahlungen den validierten Gültigkeitsbereich der Temperaturkurven überschreiten (Unterabschnitt 2.2.1), können die Temperaturangaben fehlerbehaftet sein. Wahrscheinlich ist, daß die wahren Temperaturen die gerechneten übersteigen [8]. 


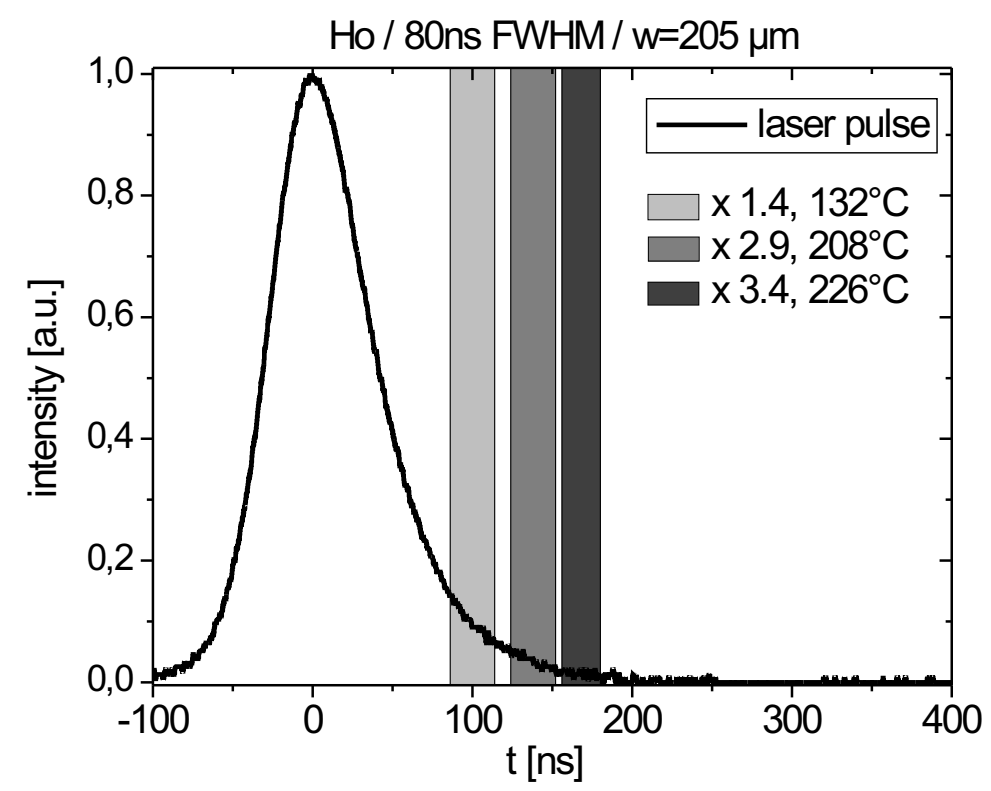

Abb. 5.2: Zeitliches Pulsprofil des gütegeschalteten Ho-Lasers und Startzeitpunkte der Ablation [9]. Die Balken markieren den mittleren Ablationsbeginn ( \pm Standardabweichung) zum angegebenen Vielfachen der Bestrahlungsschwelle $E D_{50}=131 \mathrm{~mJ} / \mathrm{mm}^{2}$. Dazu sind die Endtemperaturen angegeben, die bis zum jeweiligen Ablationsbeginn erreicht wurden. An der Schwelle ist, wie zur Tm-Laseranregung, $T\left(E D_{50}\right)=106^{\circ} \mathrm{C}$.

Frenz et al. beschreiben den Ablationsbeginn nicht über eine Häufigkeitsverteilung, sondern durch eine Reihe von Einzelmessungen mit Bestrahlungen zwischen $25 \mathrm{~mJ} / \mathrm{mm}^{2}$ und $35 \mathrm{~mJ} / \mathrm{mm}^{2}$. Die Startzeitpunkte der Ablation bewegten sich in einem Zeitfenster zwischen $0.1 \mu$ s und $0.5 \mu \mathrm{s}$ (Abbbildung 5.4). Aufgrund der zugehörigen Endtemperaturen von $38^{\circ} \mathrm{C}$ bis $45^{\circ} \mathrm{C}$ kann in diesem Fall von einem reinen Kaviationsprozeß ausgegangen werden. 


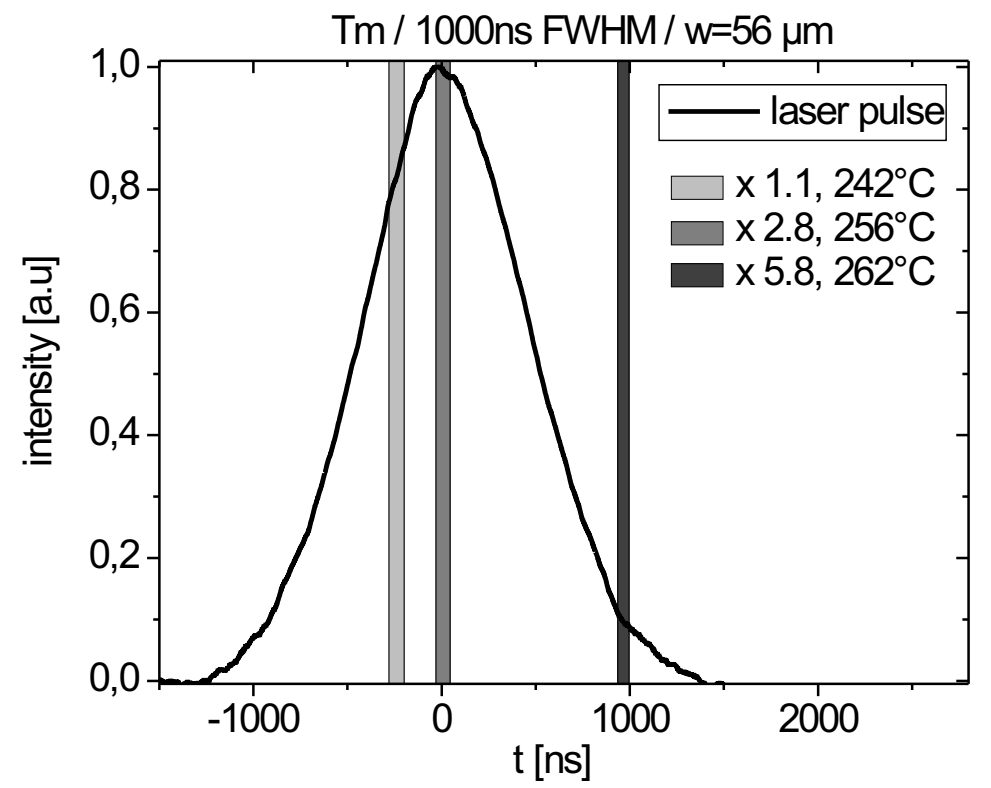

Abb. 5.3: Zeitliches Pulsprofil des freilaufenden Tm-Laserspikes und Startzeitpunkte der Ablation [9]. Die Balken markieren den mittleren Ablationsbeginn ( \pm Standardabweichung) zum angegebenen Vielfachen der Bestrahlungsschwelle $E D_{50}=338 \mathrm{~mJ} / \mathrm{mm}^{2}$. Dazu sind die Endtemperaturen angegeben, die bis zum jeweiligen Ablationsbeginng erreicht wurden. An der Schwelle ist $T\left(E D_{50}\right)=239^{\circ} \mathrm{C}$.

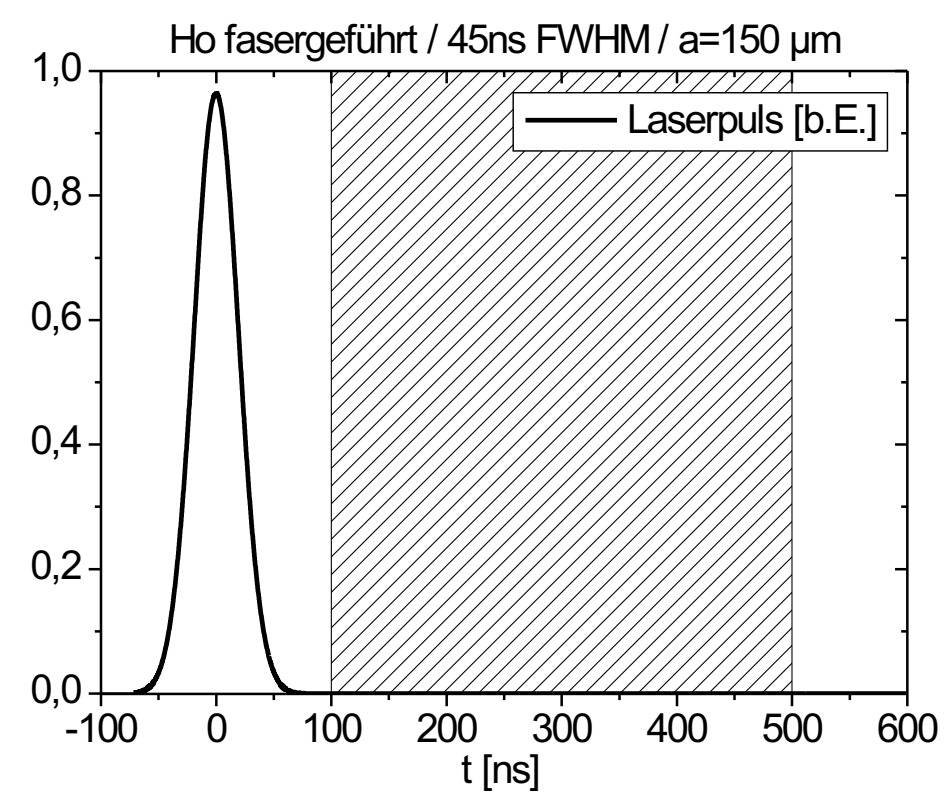

Abb. 5.4: Startzeitpunkte der Ablation (schraffierter Bereich) zur Anregung mit einem fasergeführten, gütegeschalteten Ho-Laser [24]. Bestrahlungen zwischen $25 \mathrm{~mJ} / \mathrm{mm}^{2}$ und $35 \mathrm{~mJ} / \mathrm{mm}^{2}$, mit zugehörigen Endtemperaturen von $38 \ldots 45^{\circ} \mathrm{C}$. 


\subsubsection{Ablationsbeginn und Schallentwicklung}

Für die Anregungen mit dem Gaußschen Laserstrahl sei die Schallentwicklung wieder durch die Drucktransiente im Ursprung $\vec{o}$ dargestellt, dem Ort, an dem die Maximalwerte von Druck und Temperatur sowie der zeitliche Absolutbeginn der Zugphase zu finden sind (Abschnitt 4.1). In den Abbildungen 5.5 bis 5.7 sind die experimentellen Ergebnisse um die zugehörigen Druckverläufe ergänzt. Dargestellt sind die Transienten zur jeweiligen $E D_{50}$-Bestrahlung, für höhere Bestrahlungen ändern sich im wesentlichen die Amplituden, aber weniger der Verlauf der Kurven (vgl. Abbildung 4.13).

Bei der Anregung mit den gütegeschalteten Lasern erreichen die Druckamplituden Beträge, die nahezu über die gesamte Dauer der Druckphase eine Verdampfung der Wasserprobe verhindern. Mit Dampfdrücken von 16 bar bzw. 26 bar bei den erreichten Endtemperaturen $T=201{ }^{\circ} \mathrm{C}(\mathrm{Tm})$ bzw. $T=226^{\circ} \mathrm{C}$ (Ho) ist eine Verdampfung frühestens zu den Zeitpunkten $t=90 \mathrm{~ns}$ (Ho) bzw. $t=128 \mathrm{~ns}$ (Tm) möglich. Die frühesten Ablationszeitpunkte sind mit $t=86 \mathrm{~ns}$ (Ho) bzw. $t=140 \mathrm{~ns}$ (Tm) angegeben, d.h. Modell und Experiment stimmen gut überein. Die Lage aller Ablationsstartzeitpunkte in der Zugphase des Schallfeldes weist auf eine aktive Unterstützung des Ablationsbeginns durch Kavitation hin. Haben sich in der überhitzten Flüssigkeit erste Kavitationsblasen gebildet, so bieten sie die erforderlichen Phasengrenzen zum Auslösen des Verdampfungsvorganges (Abschnitt 4.1).

Bei der Anregung mit dem freilaufenden Tm-Laserspike sind die Druckamplituden zu dieser Anregung so weit reduziert, daß die Druckphase des Schallfeldes einem Verdampfungsbeginn nicht entgegenwirkt. Hierdurch beginnt die Ablation nicht erst zum Ende des Laserpulses, sondern sobald die Probe hinreichend erhitzt ist. In den entgasten Reinstwasserproben ist hierzu eine Temperatur von ca. $250^{\circ} \mathrm{C}$ erforderlich, wie die gefundenen Endtemperaturen zeigen. Der zugehörige Dampfdruck beträgt 41 bar. Eine Unterstützung des Verdampfungsprozesses durch Kavitation kann hierbei für die beiden frühen Ablationszeitpunkte sicher ausgeschlossen werden, da sie vollständig in die Druckphase des Schallfeldes fallen. 


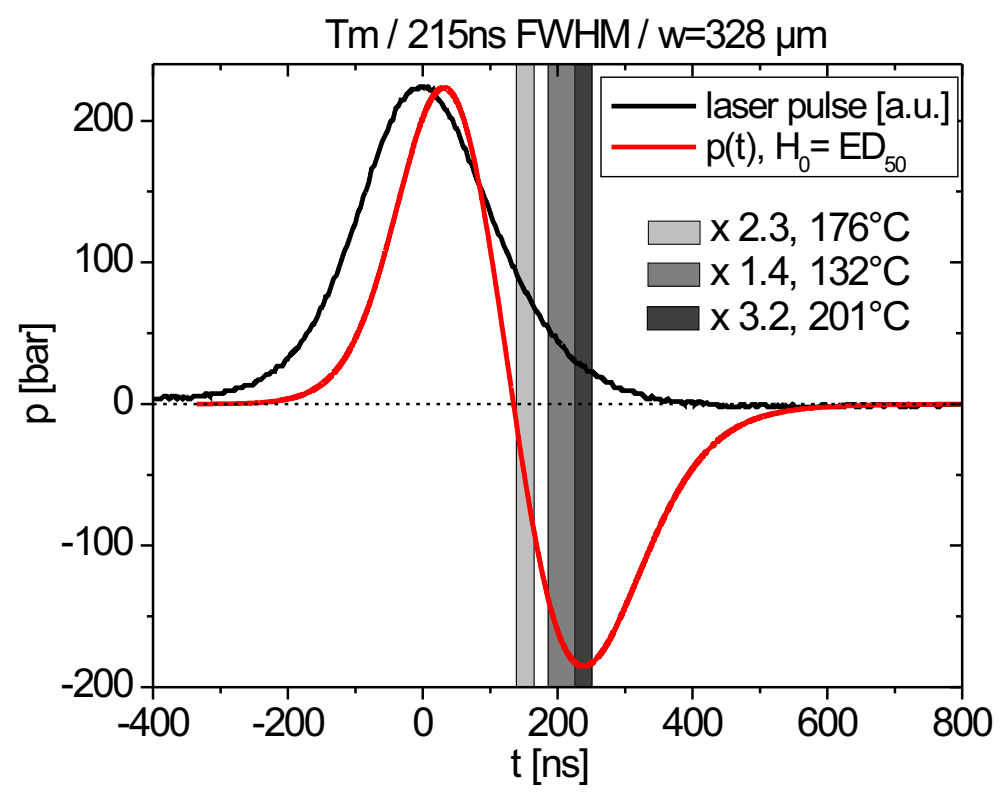

Abb. 5.5: Blasenbildung bei Anregung mit dem gütegeschalteten Thuliumlaser (ad Abbildung 5.1) und Verlauf der Drucktransiente $p(\vec{o}, t)$ zur Bestrahlung $H_{0}=E D_{50}$. Zur besseren Ansicht ist das Laserpulsprofil auf das Maximum der Transiente skaliert.

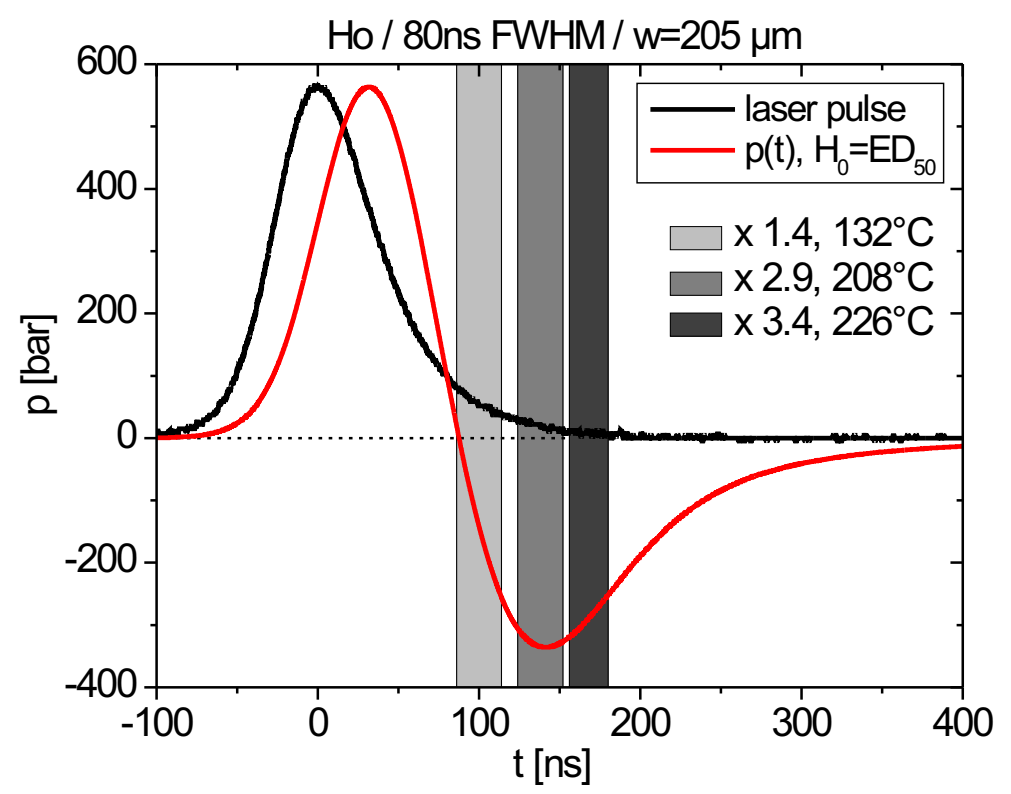

Abb. 5.6: Blasenbildung bei Anregung mit dem gütegeschalteten Holmiumlaser (ad Abbildung 5.2) und Verlauf der Drucktransiente $p(\vec{o}, t)$ zur Bestrahlung $H_{0}=E D_{50}$. Zur besseren Ansicht ist das Laserpulsprofil auf das Maximum der Transiente skaliert. 


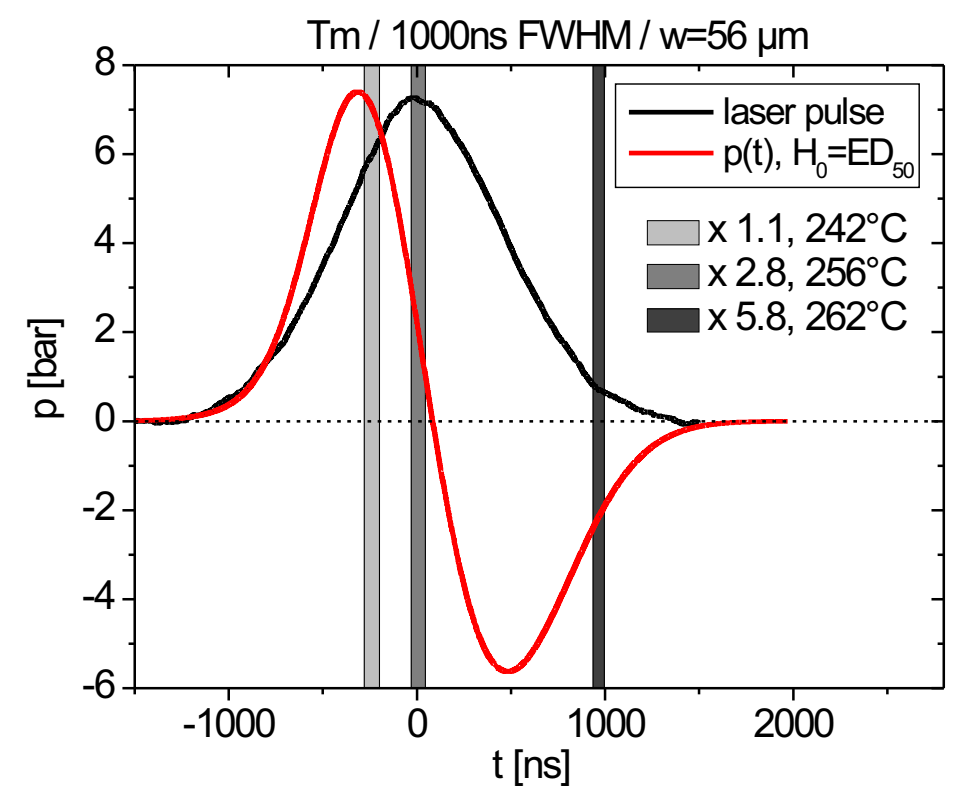

Abb. 5.7: Blasenbildung bei Anregung mit dem ersten Spike des freilaufenden Thuliumlasers (ad Abbildung 5.3) und Verlauf der Drucktransiente $p(\vec{o}, t)$ zur Bestrahlung $H_{0}=E D_{50}$. Zur besseren Ansicht ist das Laserpulsprofil auf das Maximum der Transiente skaliert.

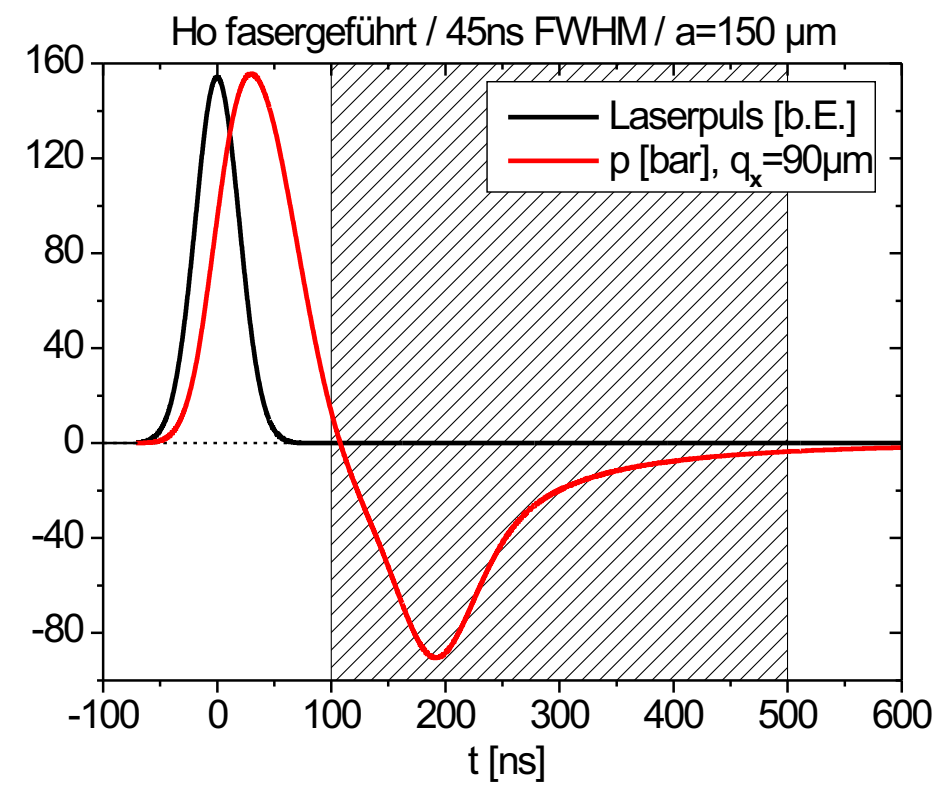

Abb. 5.8: Blasenbildung zur Anregung mit einem fasergeführten, gütegeschalteten Ho-Laser (ad Abbildung 5.4) und Verlauf der Drucktransiente $p\left(q_{x}, t\right)$ zum Radialabstand $q_{x}=90 \mu \mathrm{m}$ und der Bestrahlung $H=30 \mathrm{~mJ} / \mathrm{mm}^{2}$. 
Die von Frenz et al. beobachteten Ablationsvorgänge zur fasergeführten, gütegeschalteten Ho-Laseranregung sind aufgrund der niedrigen Endtemperaturen bereits als Kavitationsprozesse identifiziert. Um festzustellen, wo und wann die Zugphase ihren Anfang nimmt, ist zunächst die zugehörige Schallentwicklung in der Eintrittsebene berechnet worden (Abbildung 5.9). Aus dieser läßt sich ablesen, daß die ersten Zuganteile nach $t=110 \mathrm{~ns}$ in einem Radialabstand $q_{x}=90 \mu \mathrm{m}$ zur Fasermitte auftreten. Der früheste von Frenz et al. genannte Ablationsbeginn bei $t=100 \pm 20 \mathrm{~ns}$ wird hierdurch gut bestätigt. Der gesamte Verlauf der Drucktransiente $p\left(q_{x}, t\right)$ ist in Abbildung 5.8 dargestellt, zusammen mit dem Zeitfenster der gemessenen Ablationszeitpunkte. 

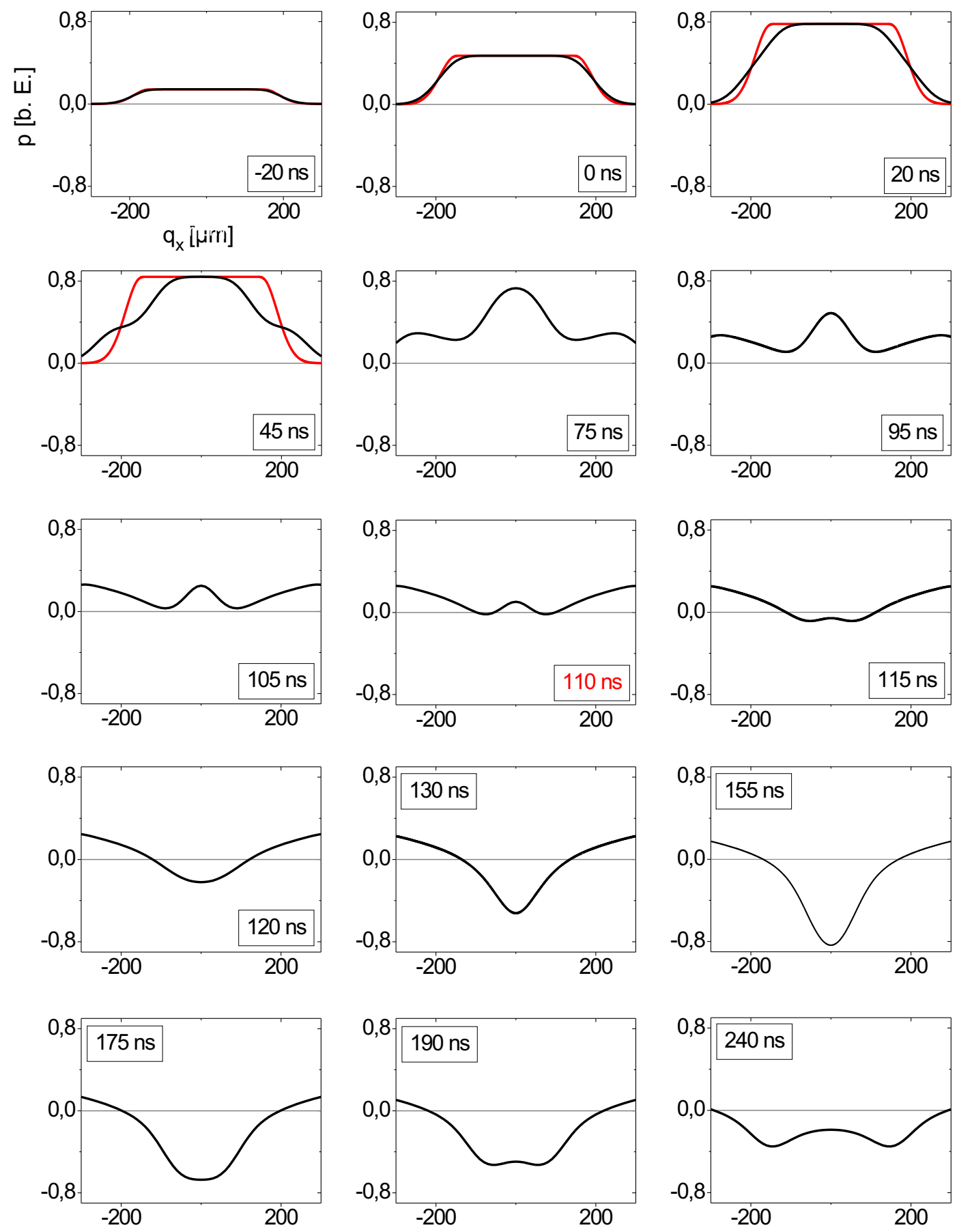

Abb. 5.9: Schallentwicklung in der Eintrittsebene zur Anregung mit dem fasergeführten, gütegeschalteten Holmiumlaser $\left(t_{\mathrm{p}}=45 \mathrm{~ns}, a=150 \mu \mathrm{m}\right)$. Dazu in Rot das Top-Hat-Strahlprofil der Laseranregung. 


\subsubsection{Schallmessungen}

Neben der optischen Detektion sind auch Messungen am Schallfeld geeignet, um den Beginn der Ablation zu erfassen. Im Schallfeld zeichnet sich der Ablationsbeginn über Druckpulse $a b$, die durch die Expansion der sich bildenden Blasen erzeugt werden $[62,63]$. Die Druckpulse überlagern die thermoelastische Schallentwicklung und führen zu sichtbaren Veränderungen gegenüber der ungestörten Schallentwicklung. Brendel und Brinkmann haben diesen Effekt für die Gaußschen Laseranregungen mit Hilfe eines PVDF-Sondenhydrophons (Durchmesser: $1 \mathrm{~mm}$ ) untersucht [9]. Das Hydrophon war in einem Mindestabstand von $4 \mathrm{~mm}$ zur Eintrittsebene des Laserstrahls ausgerichtet, um eine hinreichende Ausdehnung der Wellenfront gegenüber den Abmessungen der Hydrophonmembran zu gewährleisten (vgl. Anhang A.2). Zugleich war die Hydrophonmembran in diesem Abstand vor der Laserstrahlung und den sich bildenen Blasen geschützt. Für die gewählten Anregungsparameter befand sich das Hydrophon in der akustischen Fernfeldzone.

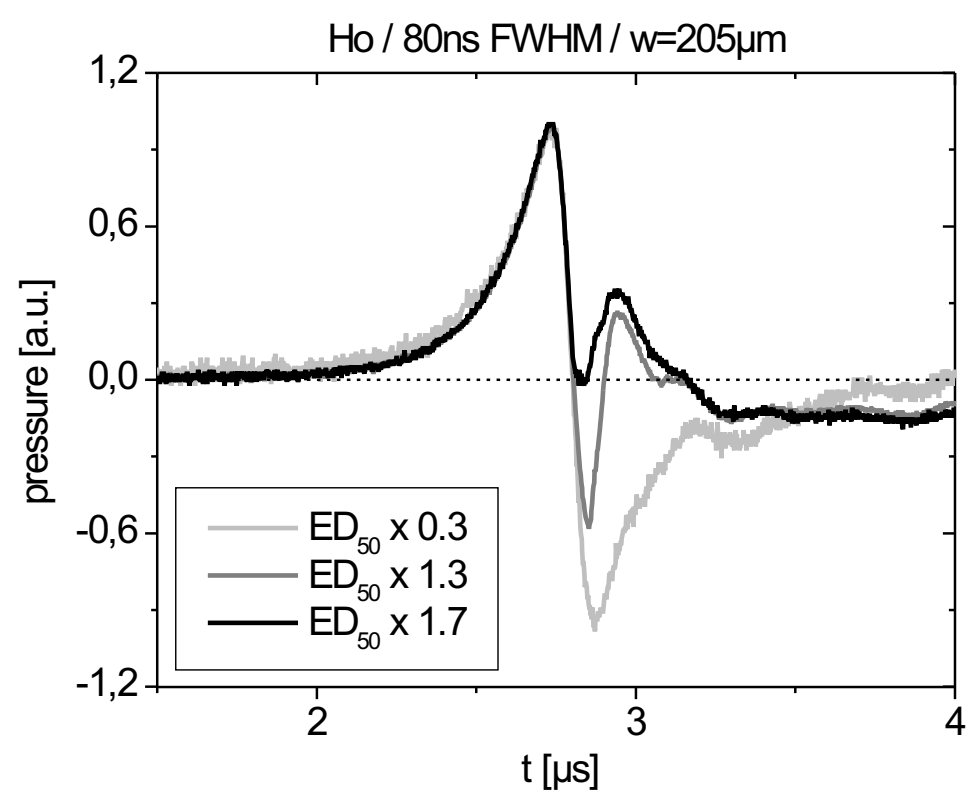

Abb. 5.10: Skalierte Fernfeldtransienten (Abstand $q_{z}=4.1 \mathrm{~mm}$ ) zur Anregung mit dem gütegeschalteten Ho-Laserpuls, gemessenen für verschiedene Bestrahlungen oberhalb und unterhalb des $E D_{50}$-Wertes [9]. Zum leichteren Vergleich der Verlaufsunterschiede sind die Kurven auf ein einheitliches Maximum skaliert.

Die Beobachtungen des Schallfeldes erfolgten zu verschiedenen Bestrahlungen unter- und oberhalb des $E D_{50}$-Wertes. Die gefundenen Veränderungen im Schallfeld (Abbildungen 


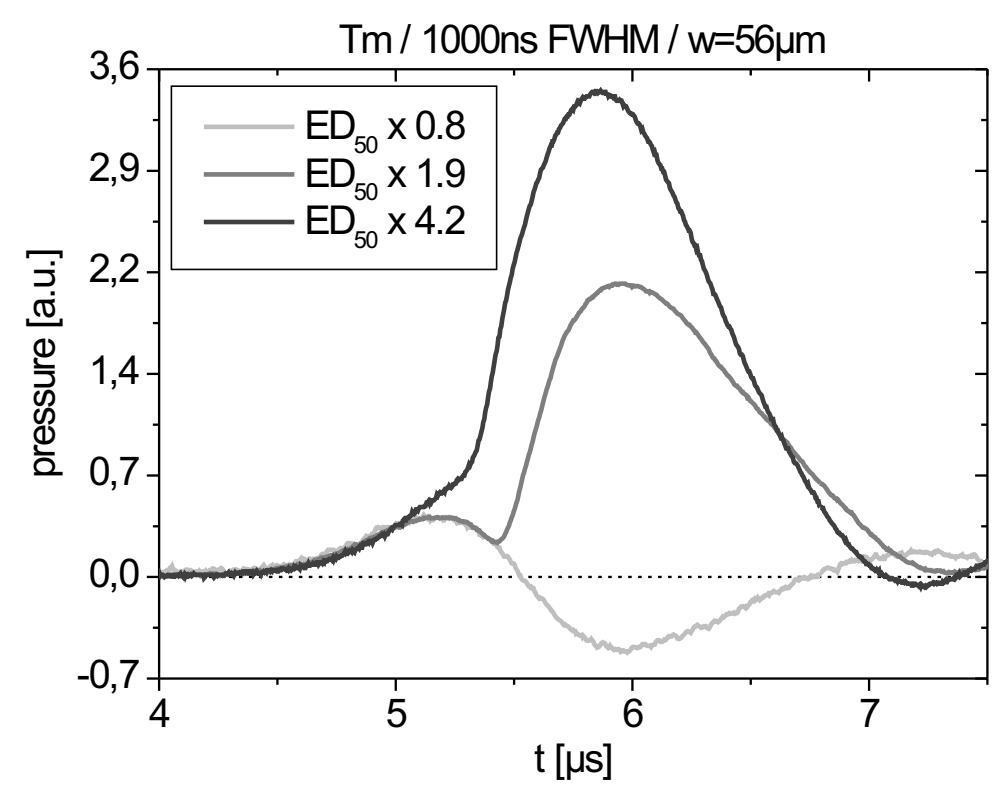

Abb. 5.11: Skalierte Fernfeldtransienten (Abstand $q_{z}=8.17 \mathrm{~mm}$ ) zur Anregung mit dem freilaufenden Tm-Laserspike, gemessenen für verschiedene Bestrahlungen oberhalb und unterhalb des $E D_{50}$-Wertes [9]. Zum leichteren Vergleich der Verlaufsunterschiede sind die Kurven auf ein einheitliches Maximum skaliert.

5.10 und 5.11) korrelieren eng mit den reflektometrisch ermittelten Ablationszeitpunkten: Zur gütegeschalteten Laser-Anregung verändert sich die Transiente immer erst in der Zugphase, zur Anregung mit dem freilaufenden Tm-Laserspike rücken die Abweichungen mit zunehmender Bestrahlung bis in die Druckphase.

Die ungestörten Fernfeldtransienten bieten die Möglichkeit eines direkten Vergleichs mit den Modellrechnungen dieser Arbeit. Da die Transienten mit einem kalibrierten Hydrophon aufgezeichnet wurden $(21 \mathrm{mV} / \mathrm{bar}$ an $10 \mathrm{pF})$, kann der Vergleich absolut erfolgen. Ausgangspunkt für die Modellrechnungen sind die Impulsantworten $p_{\delta, n}\left(q_{z}, t\right)$ (3.51), (3.52) zur axialen Schallentwicklung im Abstand $q_{z}$ von der Eintrittsebene (Unterabschnitt 3.3.1). Die resultierenden Transienten (Abbildungen 5.12 und 5.13) weisen eine weitgehende Übereinstimmung mit den gemessenen Verläufen auf. Insbesondere validiert die zeitgenaue Wiedergabe der Extrema und des Nulldurchgangs die Deutungen des Ablationsbeginns durch die zugehörige Schallentwicklung. Die Abweichungen zum Ende der Verläufe sind vermutlich auf ein gedämpftes Nachschwingen der Hydrophonmembran zurückzuführen [70]. 


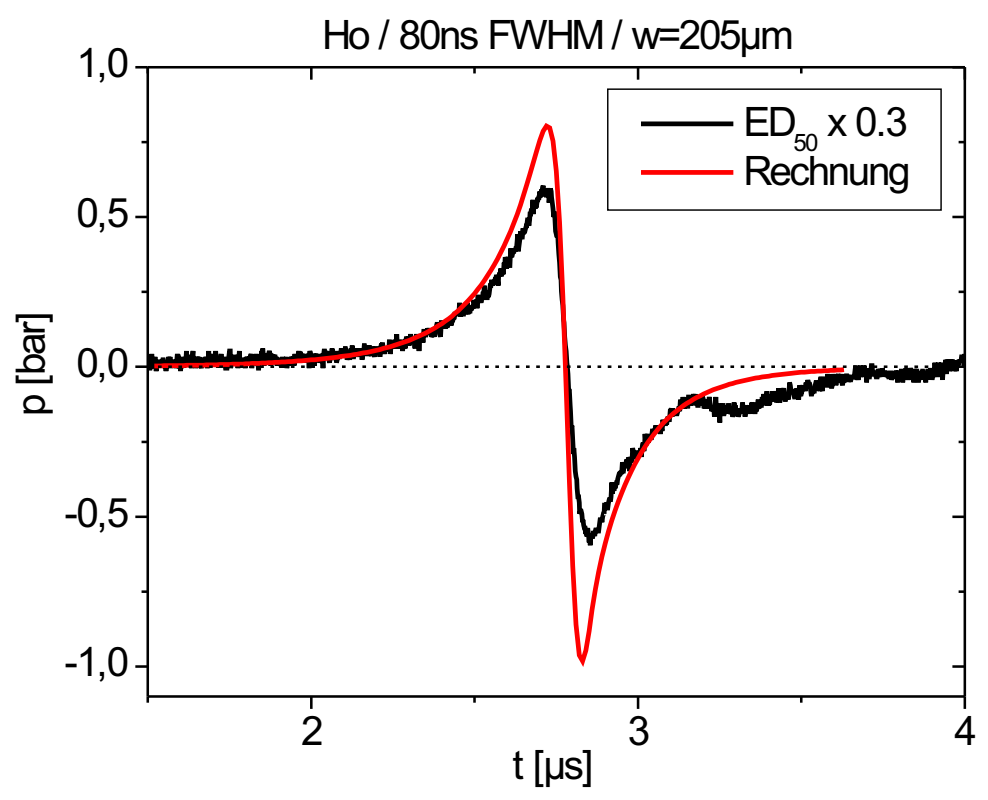

Abb. 5.12: Vergleich zwischen gerechneter und gemessener Fernfeldtransiente (ad Abbildung 5.10).

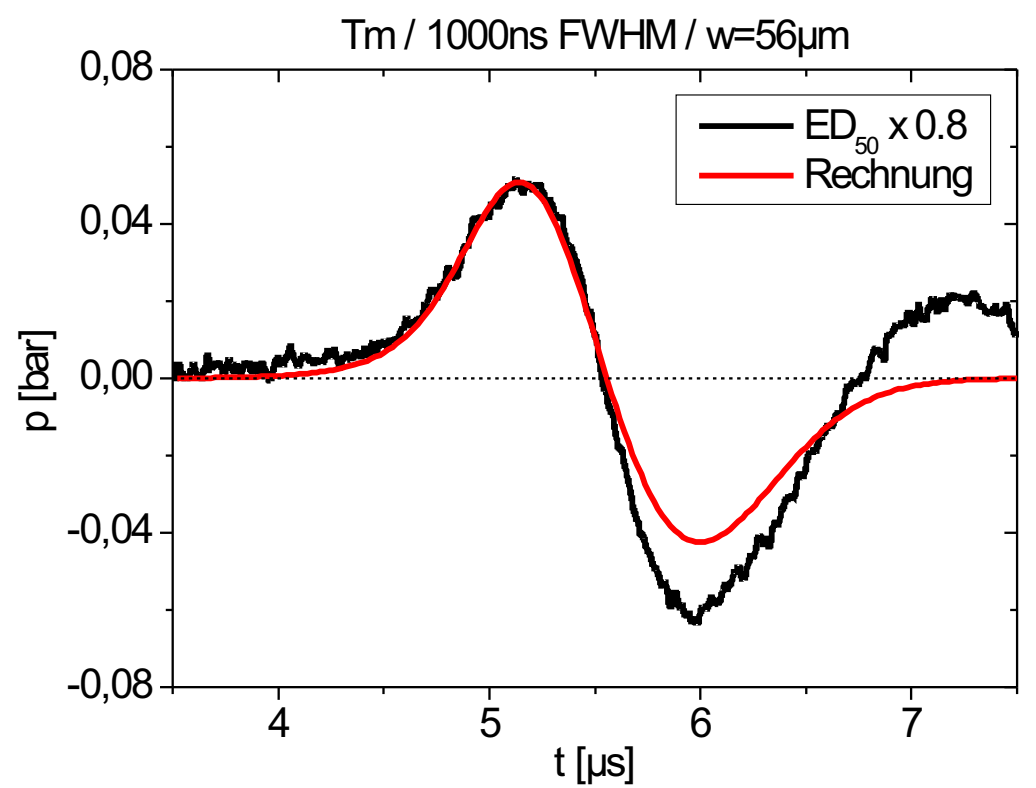

Abb. 5.13: Vergleich zwischen gerechneter und gemessener Fernfeldtransienter (ad Abbildung 5.11). 
Die deutlichsten Abweichungen zeigen sich in der Höhe der Amplituden. Sie betragen bis zu 70\% und stellen sich zudem nicht einheitlich als Über- oder Unterschätzung dar. Eine einzelne Ursache für die Unterschiede ist nicht auszumachen. Von Seiten des Modells können die Näherungsannahmen zum temperaturabhängigen Absorptionsprofil eine Rolle spielen, von experimenteller Seite sind insbesondere Meßfehler in der Pulsenergie und im Strahlradius kritisch. Eine Fehlerrechnung zeigt, daß sich ein 5\%iger Fehler im Strahlradius zusammen mit einem 10\%igen Fehler in der Pulsenergie zu einem 30\%igen Fehler in der Amplitude fortpflanzen können. Hinzu kommen ein unbekannter Kalibrierungsfehler des Hydrophons und für die Anregung zum freilaufenden Tm-Laserspike die eingeschränkte Gültigkeit der Temperaturfunktion $T(H)$ bei den verwendeten Bestrahlungen.

\section{Folgerungen für die Gewebeablation}

Die Ergebnisse zur Anregung mit dem freilaufenden Tm-Laserspike haben gezeigt: Ohne thermoelastische Schalleinwirkung setzt die Ablation ein, sobald die applizierte Bestrahlung eine zugehörige Ablationsschwelle überschritten hat. Höhere Bestrahlungen führen so zu einem früheren Ablationsbeginn, aber nicht zu einer gesteigerten Energiedeponierung in der Probe. Im Fall der Wasserproben definiert sich die Ablationsschwelle aus der jeweiligen Verdampfungstemperatur, die wiederum eine Funktion der jeweiligen Nukleationsbedingungen ist [7]. In Gewebe hängt die Verdampfungstemperatur zusätzlich vom mechanischen Einschluß der Gewebsflüssigkeit in der Gewebematrix ab [58]. Unter Einfluß des thermoelastischen Schallfeldes gelten im Prinzip die gleichen Ablationsschwellen, doch kann der Ablationsbeginn in der Druckphase temporär unterbunden werden, während er in der nachfolgenden Zugphase forciert wird. Die zeitliche Untergrenze für den Ablationsbeginn ergibt sich aus dem Ende der Druckphase, das bei der Gaußschen Laseranregung spätestens mit dem Nulldurchgang in der Drucktransiente $p(\vec{o}, t)$ erreicht ist. Da der Nulldurchgang zu fast allen betrachteten Strahlradien und Absorptionen stets nah am Pulsende liegt (Abschnitt 4.2), kann die Energie gütegeschalteter Pulse nahezu vollständig in der Probe akkumuliert werden. Dies bietet nicht nur die Möglichkeit, hohe Temperaturen und damit hohe Denaturierungsraten im bestrahlten Gewebe zu erzeugen, sondern auch, äußerst energiereiche Verdampfungsprozesse zu realisieren. Dieser Mechanismus bleibt nicht auf die betrachteten Tm- und Ho-Laseranwendungen beschränkt, sondern gilt für alle Anwendungen mit vergleichbaren Bestrahlungsbedingungen. 


\section{Zusammenfassung}

Die gepulste IR-Laserablation in Wasser ist durch simultane Temperatur- und Druckänderungen gekennzeichnet. Die Druckänderungen basieren auf dem thermoelastischen Effekt und führen zur Ausbildung eines Schallfeldes. Im Rahmen dieser Arbeit wurde ein Modell entwickelt, das Entstehung und Ausbreitung des thermoelastischen Schallfeldes als Funktion der Anregungsbedingungen wiedergibt. Zusammen mit einer Modellierung der Temperaturentwicklung läßt sich so der thermodynamische Zustandsverlauf des angeregten Probevolumens nachzeichnen und insbesondere der Zeitpunkt des verdampfungsoder kavitationsbedingten Phasenübergangs untersuchen.

Die Modellierung erfolgte am Beispiel der Anregung mit gütegeschalteten Thuliumund Holmium-Laserpulsen (Cr:Tm:YAG, $\lambda=2.01 \mu \mathrm{m}$ und Cr:Tm:Ho:YAG, $\lambda=2.1 \mu \mathrm{m}$ ). Beide Lasersysteme weisen bei den zugehörigen Laserwellenlängen eine moderate, temperaturabhängige Absorption in Wasser auf. Die Temperaturabhängigkeit der Absorptionen hat wesentlichen Einfluß auf die Temperaturentwicklung und ist entsprechend in der Temperatur-Modellierung berücksichtigt.

Grundlage des thermoelastischen Schallmodells ist die photoakustische Wellengleichung, eine lineare, inhomogene Wellengleichung, deren Inhomogenität durch das zeitliche und räumliche Anregungsprofil des Lasers gegeben ist. Zur Integration der photoakustischen Wellengleichung wurde ein Separationsansatz gewählt, der auf die Poisson'sche Formel aus der Akustik zurückführt. Die Poisson'sche Formel ist ein 3-dimensionaler Lösungsansatz, der akustische Beugungseffekte, etwa die Entstehung von Zuganteilen aus der initialen Druckerhöhung, korrekt darstellt. Weiter ist die Temperaturabhängigkeit des Grüneisenkoeffizienten in der Lösung berücksichtigt. Der Grüneisenkoeffizient stellt die Proportionalität zwischen eingebrachter Strahlungsenergie und resultierenden Druckerhöhung her. Seine Temperaturabhängigkeit vermittelt thermische Nichtlinearitäten, die Amplitude und Form des Schallfeldes beeinflussen, ohne dabei die Linearität der Schallausbreitung $\mathrm{zu}$ verletzen. 
Die Modellierungen der Schallentwicklung erfolgten für das Top-hat-Profil eines fasergeführten Laserstrahls und für ein Gaußsches Strahlprofil. Durch Darstellung der Schallentwicklung in der Eintrittsebene der Laserstrahlen konnte nachgewiesen werden, daß für den Gaußschen Laserstrahl der Übergang von der Druck- zur Zugphase des Schallfeldes stets in der Strahlmitte beginnt. Der Verlauf der Drucktransiente an diesem Punkt bestimmt somit den frühestmöglichen Kavitationsbeginn in der angeregten Wasserprobe. Übersteigen die Amplituden der Druckphase zudem den Dampfdruck der erhitzten Probe, so ist vor Ende der Druckphase keine Verdampfung möglich. Der Nulldurchgang der bipolaren Transiente sowie die Höhe von Druck- und Zugamplitude charakterisieren somit den gesamten thermodynamischen Zustandsverlauf der angeregten Probe. Die Größen werden im folgenden als charakteristische Schallfeldgrößen bezeichnet.

Die charakteristischen Schallfeldgrößen wurden auf ihre Abhängigkeit von den Bedingungen einer Gaußschen Laseranregung untersucht. Die Anregungsbedingungen sind durch Laserpulsdauer, Pulsenergie, Strahlradius und Absorption festgelegt. Eine wesentliche Vereinfachung der Untersuchungen ergab sich dadurch, daß im Rahmen dieser Arbeit eine analytische Lösung der Poisson'schen Formel für alle Punkte der Gaußschen Laserstrahlachse gefunden werden konnte. Bisherige Lösungen beschränkten sich in ihrer Gültigkeit auf das akustische Fernfeld, während die vorliegende auch im akustischen Nahfeld gilt und somit insbesondere die Drucktransiente zu den charakteristischen Schallfeldgrößen abdeckt.

Aus den gefundenen charakteristischen Schallfeldgrößen läßt sich ableiten, daß bei den Pulsdauern der gütegeschalteten Laseranregungen stets mit einer durch die Druckphase verzögerten Verdampfung gerechnet werden muß. Mit zunehmenden Pulsenergien können so zunehmende Probentemperaturen erreicht werden, die nachfolgend zu einem explosiven Verdampfungsprozeß weit oberhalb des normalen Siedepunktes führen. Ausgelöst wird der Verdampfungsprozeß spätestens durch Kavitation in der Zugphase des Schallfeldes. Die Ablationswirkung eines solchen Prozesses ist deutlich von der zu einer stetigen Verdampfung bei kontinuierlicher Probenanregung zu unterscheiden. Die vorhergesagten Ablationszeitpunkte werden durch Vergleich mit experimentellen Beobachtungen gut bestätigt.

Bei der fasergeführten Laseranregung zeigt die Modellierung ein besonderes Verhalten des Schallfeldes: Anders als zum Gaußschen Laserstrahl ist der Übergang von der Druckzur Zugphase nicht ortsfest, sondern variiert mit der Pulsdauer zwischen Strahlmitte und den Flanken des Strahlprofils. Eine allgemeine Analyse der Schallfeldentwicklung 
als Funktion der Anregungsbedingungen ist hierdurch nicht möglich. Vielmehr ist die Schallfeldentwicklung individuell zur jeweiligen Anregungssituation zu berechnen und hieraus der zugehörige thermodynamische Zustandsverlauf abzuleiten. Durch exemplarischen Vergleich mit dem Experiment ist dieses Modellergebnis ebenfalls gut bestätigt.

Die Modellvorhersagen zu den laserinduzierten Zustandsänderungen in Wasser sind im Grundsatz direkt auf Gewebe übertragbar. Zwar erhöht der mechanische Einschluß der Gewebsflüssigkeit in der Gewebematrix die Ablationsschwellen, doch wirkt das thermoelastische Schallfeld in gleicher Weise auf den Verdampfungsprozeß ein. Es hängt von der medizinischen Indikation ab, ob die Probe z.B. gezielt überhitzt werden soll, um durch thermische Denaturierung, explosive Verdampfung und ausgeprägte Zugwellen einen breiten Wundreiz zu erzeugen, oder ob vielleicht ein kontrollierter Verdampfungsvorgang mit geringen Abtragsraten aber hoher Präzision erwünscht ist. Daß sich zu den Anregungsbedingungen der gütegeschalteten Tm- und Ho-Laseranregung vorwiegend das explosive Ablationsregime einstellt, ist ein zentrales Vorhersageergebnis der durchgeführten Modellrechnungen. 


\section{A. Anhang}

\section{A.1. Lösung der Poisson'schen Formel-Integrale}

In den Berechnungen zur Schallentwicklung auf der Strahlachse, vgl. Unterabschnitt 3.3.1, wurde die Lösung der beiden Teilintegrale

$$
\begin{aligned}
& J_{-}(r, n) \equiv t \int_{0}^{\vartheta_{\mathrm{G}}} \sin (\vartheta) \exp \left(-2 n \frac{r^{2} \sin ^{2}(\vartheta)}{w^{2}}-\bar{\mu} n r \cos (\vartheta)\right) \mathrm{d} \vartheta \quad \text { und } \\
& J_{+}(r, n) \equiv t \int_{\vartheta_{\mathrm{G}}}^{\pi} \sin (\vartheta) \exp \left(-2 n \frac{r^{2} \sin ^{2}(\vartheta)}{w^{2}}+\bar{\mu} n r \cos (\vartheta)\right) \mathrm{d} \vartheta
\end{aligned}
$$

als Kernproblem bei der Lösungsbestimmung der Poisson'schen Formel zum $n$-ten Quelltermglied herausgearbeitet. Der Grenzwinkel $\Theta_{\mathrm{G}}$ war hierbei durch Gl. (3.28),

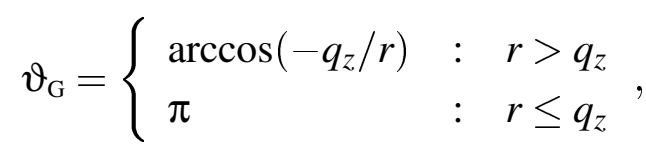

bestimmt. Gegenstand dieses Abschnittes ist es darzustellen, wie die beiden Integrale gelöst werden können, indem sie auf die imaginäre Fehlerfunktion

$$
\operatorname{erfi}(\zeta(r))=\frac{2}{\sqrt{\pi}} \int_{0}^{\zeta(r)} \mathrm{e}^{\tau^{2}} \mathrm{~d} \tau
$$

zurückgeführt werden (mit einer beliebigen linearen Funktion $\zeta(r)$ ). Bei der Lösungsbestimmung sind hierbei die beiden Fälle $r>q_{z}$ und $r \leq q_{z}$ zum Grenzwinkel $\vartheta_{\mathrm{G}} \mathrm{zu}$ unterscheiden:

Fall $\mathbf{r}>\mathbf{q}_{\mathbf{z}}$

Die einzelnen Lösungsschritte werden zunächst für das Integral $J_{-}(r, n)$ erläutert. Nach 
Definition der beiden Hilfsvariablen $\gamma \equiv 2 n r^{2} / w^{2}$ und $s \equiv \bar{\mu} n r$ und unter Ausnutzung von $\sin ^{2}(\vartheta)=1-\cos ^{2}(\vartheta)$ erhält das Integral die Form

$$
\left.J_{-}(r, n)\right|_{r>q_{z}}=t \int_{0}^{\vartheta_{\mathrm{G}}} \sin (\vartheta) \exp \left(-\gamma\left\{1-\cos ^{2}(\vartheta)\right\}-s \cos (\vartheta)\right) \mathrm{d} \vartheta .
$$

Die nächste Umformung erfolgt mit Hilfe der Variablensubstitution $y \equiv \sqrt{\gamma} \cos (\vartheta)$. Bei dieser Substitution wird ausgenutzt, daß der Vorfaktor $\sin (\vartheta)$ im Integranden über die Ableitung $\mathrm{d} y / \mathrm{d} \vartheta$ eliminiert werden kann. Es ist $\mathrm{d} \vartheta \sin (\vartheta)=-\mathrm{d} y / \sqrt{\gamma}$ und damit

$$
\begin{aligned}
\left.J_{-}(r, n)\right|_{r>q_{z}} & =-t \frac{\exp (-\gamma)}{\sqrt{\gamma}} \int_{\sqrt{\gamma}}^{\sqrt{\gamma} \cos \left(\vartheta_{\mathrm{G}}\right)} \exp \left(y^{2}-\frac{s y}{\sqrt{\gamma}}\right) \mathrm{d} y \\
& =-t \frac{\exp \left(-\left(\gamma+\frac{s^{2}}{4 \gamma}\right)\right)}{\sqrt{\gamma}} \int_{\sqrt{\gamma}}^{\sqrt{\gamma} \cos \left(\vartheta_{\mathrm{G}}\right)} \exp \left(\left(y-\frac{s}{2 \sqrt{\gamma}}\right)^{2}\right) \mathrm{d} y .
\end{aligned}
$$

Der Integrand besteht nun bereits aus der positiv-quadratischen Exponentialfunktion, wie sie auch in der imaginären Fehlerfunktion auftritt. Die nächste Variablensubstitution lautet entsprechend $z \equiv y-s /(2 \sqrt{\gamma}) \rightarrow \mathrm{d} z=\mathrm{d} y$ und führt auf

$$
\left.J_{-}(r, n)\right|_{r>q_{z}}=t \frac{\exp \left(-\left(\gamma+\frac{s^{2}}{4 \gamma}\right)\right)}{\sqrt{\gamma}} \int_{a(r)}^{b\left(q_{z}\right)} \exp \left(z^{2}\right) \mathrm{d} z
$$

Die Integrationsgrenzen sind hierbei durch die Funktionen

$$
\begin{aligned}
& b(r, n)=\sqrt{n}\left(\frac{\sqrt{2} r}{w}+\frac{\bar{\mu} w}{\sqrt{8}}\right) \text { und } \\
& a(r, n)=\sqrt{n}\left(\frac{\bar{\mu} w}{\sqrt{8}}-\frac{\sqrt{2} r}{w}\right)
\end{aligned}
$$

bestimmt. Das negative Vorzeichen vor dem gesamten Integral ist in diese Funktionen eingeflossen. Durch Rückersetzen der beiden Hilfsvariablen $\gamma$ und $s$ ergibt sich die Enddarstellung des Integrals:

$$
\left.J_{-}(r, n)\right|_{r>q_{z}}=\frac{w}{c_{0}} \sqrt{\frac{\pi}{8 n}} \exp \left(-n\left(\frac{2 r^{2}}{w^{2}}+\frac{\bar{\mu}^{2} w^{2}}{8}\right)\right)\left[\operatorname{erfi}\left(b\left(q_{z}, n\right)\right)-\operatorname{erfi}(a(r, n))\right] .
$$


Die Berechnung des Integrals $J_{+}(r, n)$ erfolgt analog zu $J_{-}(r, n)$ unter Berücksichtigung des veränderten Vorzeichens im Integranden. Dies führt auf die Enddarstellung

$$
\left.J_{+}(r, n)\right|_{r>q_{z}}=\frac{w}{c_{0}} \sqrt{\frac{\pi}{8 n}} \exp \left(-n\left(\frac{2 r^{2}}{w^{2}}+\frac{\bar{\mu}^{2} w^{2}}{8}\right)\right)\left[\operatorname{erfi}\left(a\left(q_{z}, n\right)\right)-\operatorname{erfi}(a(r, n))\right] .
$$

Fall $\mathbf{r} \leq \mathbf{q}_{\mathbf{z}}$

In diesem Fall ist $\vartheta_{\mathrm{G}}=\pi$ und damit $J_{+}(r, n)=0$. Das Integral $J_{-}(r, n)$ berechnet sich in analoger Weise zum vorherigen Fall. Es ergibt sich die Enddarstellung:

$$
\left.J_{-}(r, n)\right|_{r \leq q_{z}}=\frac{w}{c_{0}} \sqrt{\frac{\pi}{8 n}} \exp \left(-n\left(\frac{2 r^{2}}{w^{2}}+\frac{\bar{\mu}^{2} w^{2}}{8}\right)\right)[\operatorname{erfi}(b(r, n))-\operatorname{erfi}(a(r, n))] .
$$

Die Lösungsdarstellung der Integrale ist damit vollständig.

\section{A.2. Geometrische Betrachtungen zur Schalldetek- tion}

Bei der zeitaufgelösten Messung einer Wellenfront stellt sich die Aufgabe, geometrische Laufzeiten über die räumlich augedehnte Detektorfläche zu minimieren. Läuft z.B. eine ebene Wellenfront schräg über die Detektorfläche hinweg, so führt dies zu einer künstlichen Streckung des Zeitsignals bei einer gleichzeitigen Verringerung der Signalamplitude. Verstärkt wird dieses Artefakt, wenn die Wellenfront eine Krümmung aufweist, deren Radius klein gegenüber den Detektorabmessungen ist. Der schräge Wellenlauf über den Detektor kann durch parallele Ausrichtung des Detektors zur Wellenfront vermieden werden. Das Verhältnis von Krümmungsradius zu Detektorgröße läßt sich durch größere Abstände zur Schallquelle verbessern, da der Krümmungsradius einer Welle stetig mit der Ausbreitung zunimmt.

Die Form, mit der sich die thermoelastische Schallwelle ausbreitet, ist ein Abbild der optischen Zone: Aus einer breiten, flachen Zone $(\mu w \rightarrow \infty)$ entsteht eine ebene Wellenform, für eine schmale, lange Zone $(\mu w \rightarrow 0)$ nimmt die Welle ein zylindrisches Profil an. Dazwischen, für $(\mu w \approx 1)$, ist die Ausbreitung sphärisch [38]. Die Detektion einer sphärischen Wellenfront ist in Abbildung A.1 dargetellt. Sie zeigt ein Hydrophon vom Durchmesser $d$, das in einem Abstand $z_{0}$ von der Grenzfläche aufgestellt ist. Wie gefordert ist das Hydrophon parallel zur Wellenfront ausgerichtet. Die Abbildung illlustriert den Lauf der Wellenfront über das Hydrophon: Er beginnt ab einem Sphärenradius $r=z_{0}$ 


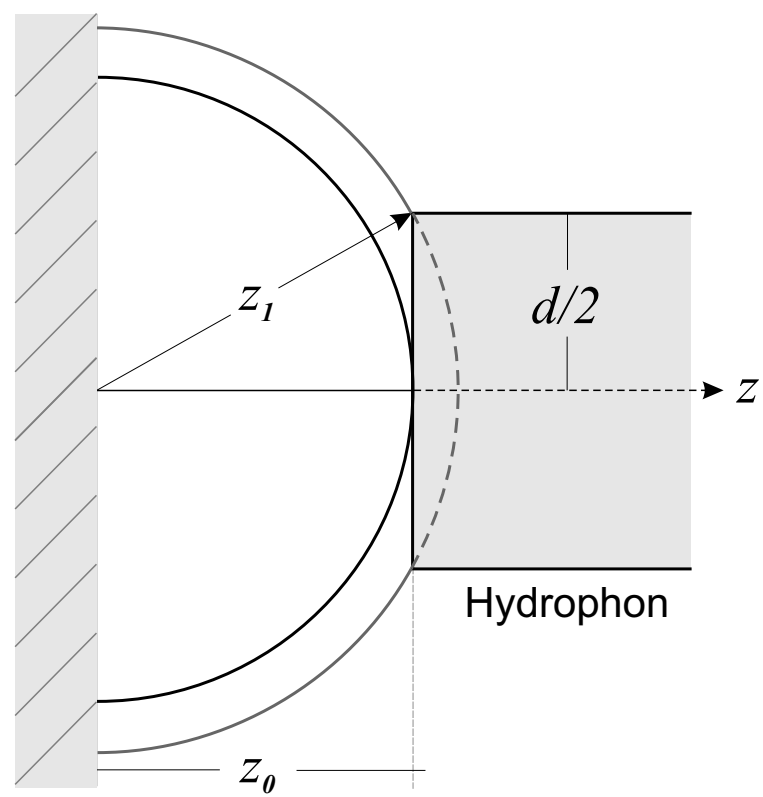

Abb. A.1: Schalldetektion einer sphärischen Wellenfront mit einem Hydrophon vom Durchmesser $d$, im Abstand $z_{0}$ von der Grenzfläche.

in der Hydrophonmitte und endet zum Radius $z_{1}=\sqrt{z_{0}^{2}+(d / 2)^{2}}$ an dessen Rand. Der gesamte Vorgang erstreckt sich über einen Zeitraum $T$, der sich mit der Schallgeschwindigkeit $c_{0} \mathrm{zu}$

$$
T\left(z_{0}\right)=\frac{z_{1}-z_{0}}{c_{0}}=\frac{\sqrt{z_{0}^{2}+(d / 2)^{2}}-z_{0}}{c_{0}}
$$

ergibt und erwartungsgemäß für $z_{0} \rightarrow \infty$ gegen Null strebt. Die Messung kann für endliche Abstände $z_{0}$ als ungestört angenommen werden, wenn $T\left(z_{0}\right)$ kurz ist im Vergleich zum eigentlichen Meßsignal, dem Druckverlauf $p(t)$ in der Wellenfront. Der Verlauf $p(t)$ sei durch eine obere Grenzfrequenz $f_{\mathrm{G}}$ charakterisiert. Dann lautet die Bedingung für eine störungsfreie Messung $T\left(z_{0}\right) \ll 1 / f_{\mathrm{G}}$, die sich mit Gl. (A.11) zu einer äquivalenten Forderung an den Abstand des Detektors zur Schallquelle umformen läßt:

$$
z_{0} \gg \frac{(d / 2)^{2}-\left(c_{0} f_{\mathrm{G}}\right)^{2}}{2 c_{0} f_{\mathrm{G}}}
$$

Die Ungleichung sei am Beispiel einer typischen Ho-Laseranregung $\left(t_{\mathrm{p}}=80 \mathrm{~ns}, \mu=\right.$ $3 \mathrm{~mm}, w=200 \mu \mathrm{m})$ quantifiziert: Aus der Fourieranalyse $\mathcal{F}[p(\vec{o}, t)]$ des zugehörigen Druckverlaufs errechnet sich die obere Grenzfrequenz (3dB-Abfall) zu $f_{\mathrm{G}}=15 \mathrm{MHz}$. Bei einem angenommenen Detektordurchmesser $d=1 \mathrm{~mm}$ ergibt sich damit die Forderung $z_{0} \gg 1.2 \mathrm{~mm}$ für die Positionierung des Detektors im Experiment. 


\section{A.3. Wärmeleitung an der Grenzfläche Quarz- Wasser}

Bei der IR-Laserablation an einer freien Oberfläche (z.B. Haut) wirkt die Umgebungsluft als effektiver thermischer Isolator, so daß während der typischen Bestrahlungszeiträume von einigen Hundert Nano- bis Mikrosekunden praktisch keine Wärme an die Umgebung abfließt [58]. Bei der fasergeführten Laseranregung findet hingegen eine merkliche Wärmeableitung in den aufgesetzten Quarzkörper statt. Die Wärmeleitfähigkeit $k$ $[\mathrm{W} / \mathrm{mK}]$ und der Temperaturleitwert $\gamma\left[\mathrm{m}^{2} / \mathrm{s}\right]$ von synthetischem Quarz und dazu vergleichend von Wasser und Luft sind in Tabelle A.1 aufgelistet.

\begin{tabular}{|l|l|l|}
\hline & $k[\mathrm{~W} / \mathrm{mK}]$ & $\gamma\left[\mathrm{m}^{2} / \mathrm{s}\right]$ \\
\hline \hline syn. Quarz & 1.38 & $8.122 \cdot 10^{-7}$ \\
\hline Wasser & 0.609 & $1.46 \cdot 10^{-7}$ \\
\hline Luft & 0.025 & $1.94 \cdot 10^{-5}$ \\
\hline
\end{tabular}

Tabelle A.1: Wärmeleitfähigkeit $k$ und Temperaturleitweit $\gamma$ für die Materialien synthetischer Quarz, Wasser und Luft [75, 26, 84].

Zur Modellierung des Wärmeflusses seien die Wasserprobe und der eingetauchte Quarzkörper in erster Näherung als semi-infinite Körper angenommen, zwischen denen der Wärmeaustausch entlang einer Achse $z$ stattfindet, die senkrecht zur Grenzfläche beider Medien steht und in Richtung Wasser weist. Die Grenzfläche befinde sich bei $z=0$. Die Größe $\Theta_{i}(t, z) \equiv T-T_{0}$ stelle die Temperaturerhöhung jeweils im Wasser $(i=1)$ und im Quarz $(i=2)$ dar. Da auf den betrachteten Zeitskalen Konvektionsvorgänge im Wasser vernachlässigbar sind, gilt in beiden Medien die Wärmeleitungsgleichung [84]

$$
\frac{\partial \Theta_{i}}{\partial t}=\gamma_{i} \frac{\partial^{2} \Theta_{i}}{\partial z^{2}}
$$

Hierbei steht $\gamma_{i}$ für den jeweiligen Temperaturleitwert der beiden Medien gemäß Tabelle A.1. An der Grenzfläche gelten die Randbedingungen

$$
\begin{aligned}
& \Theta_{1}(t, z=0)=\Theta_{1}(t, z=0), \quad \text { sowie } \\
& \left.k_{1} \frac{\partial \Theta_{1}}{\partial z}\right|_{z=0}=\left.k_{2} \frac{\partial \Theta_{2}}{\partial z}\right|_{z=0},
\end{aligned}
$$

worin $k_{i}$ die jeweilige Wärmeleitfähigkeit ist. Zudem wird

$$
\lim _{z \rightarrow \infty} \Theta_{1}(t, z)=\lim _{z \rightarrow \infty} \Theta_{2}(t, z)=0
$$


gefordert. Zur Lösung der Wärmeleitungsgleichung (A.13) unter den gegebenen Randbedingungen sei zunächst der Fall einer instantanen Laseranregung angenommen, durch die das axiale Temperaturprofil

$$
\left.\Theta(t, z)\right|_{t=0}=\Theta_{0} \exp (-\mu z)
$$

im Wasser erzeugt wird. Hierbei ist $\mu$ der Absorptionskoeffizient und $\Theta_{0}$ die Temperaturerhöhung am Eingang der Wasserprobe. A. Yablon et al. haben für diesen Fall eine anaytische Lösung bestimmt, deren Richtigkeit durch Vergleich mit einem Finite-ElementeModell überprüft wurde [84]. Danach ist die zeitliche Entwicklung des axialen Temperaturprofils im Wasser durch eine Impulsantwort $\Theta_{\delta}(t, z)$ von der folgenden Form gegeben:

$$
\Theta_{\delta}(t, z)=\frac{\Theta_{0}}{2} \exp \left(\mu^{2} \gamma_{1} t\right)\left[\exp (-\mu z) G_{1}(t, z)+\exp (+\mu z) K G_{2}(t, z)\right]
$$

Die Größen $G_{1}, G_{2}$ und $K$ sind dabei wie folgt definiert:

$$
\begin{aligned}
G_{1}(t, z) & =1+\operatorname{erf}\left(\frac{z}{2 \sqrt{\gamma_{1} t}}-\mu \sqrt{\gamma_{1} t}\right) \\
G_{2}(t, z) & =1-\operatorname{erf}\left(\frac{z}{2 \sqrt{\gamma_{1} t}}+\mu \sqrt{\gamma_{1} t}\right) \text { und } \\
K & =\frac{k_{1}-k_{2} \sqrt{\gamma_{1} / \gamma_{2}}}{k_{1}+k_{2} \sqrt{\gamma_{1} / \gamma_{2}}} .
\end{aligned}
$$

Aufgrund der Linearität der Wärmeleitungsgleichung (A.13) lassen sich alle Fälle einer zeitlich ausgedehnten Laseranregung mit Hilfe der Impulsantwort $\Theta_{\delta}(t, z)$ beschreiben. Der zugehörige Temperaturverlauf $\Theta(t, z)$ berechnet sich aus der Faltung

$$
\left[\Theta_{\delta}(\tau, z) \otimes g(\tau)\right](t)=\int_{-\infty}^{\infty} \Theta_{\delta}(t-\tau, z) g(\tau) \mathrm{d} \tau
$$

der Impulsantwort mit dem Laserpulsprofil $g(t)$, das beliebig gewählt werden kann, aber $\mathrm{zu}$

$$
\int_{-\infty}^{\infty} g(\tau) d \tau=1
$$

normiert sein muß. Diese Normierungsbedingung verhindert, daß die Leistung des Laserpulses doppelt in die Berechnungen eingeht, da sie bereits verwendet wird, um die maximale Temperaturerhöhung $\Theta_{0}$ in der Impulsantwort zu bestimmen. Gemäß 2.2.1 geschieht 
dies über den Zusammenhang

$$
\mathrm{d} T=\frac{\mu}{\rho C_{\mathrm{V}}} I_{0}(t) \mathrm{d} t
$$

worin $I_{0}(t)$ die Leistung am Eingang der Wasserprobe ist.

Das Verhalten des dargestellten Wärmeleitungssystems läßt sich durch eine Analyse der Impulsantwort $\Theta_{\delta}(t, z)$ zum Zeitpunkt $t=0$ grob vorhersagen: Für die Unterfunktionen $G_{1,2}(t, z)$ ergeben sich bei $t=0$ die Werte

$$
\begin{aligned}
& G_{1}(0, z)=1+\operatorname{erf}(\infty)=2, \quad \text { sowie } \\
& G_{2}(0, z)=1-\operatorname{erf}(\infty)=0,
\end{aligned}
$$

womit die Impulsantwort von folgender Form ist:

$$
\Theta_{\delta}(0, z)=\left\{\begin{array}{ccc}
\frac{1+K}{2} \Theta_{0} & \text { für } & z=0 \\
\Theta_{0} \exp (-\mu z) & \text { für } & z>0 .
\end{array}\right.
$$

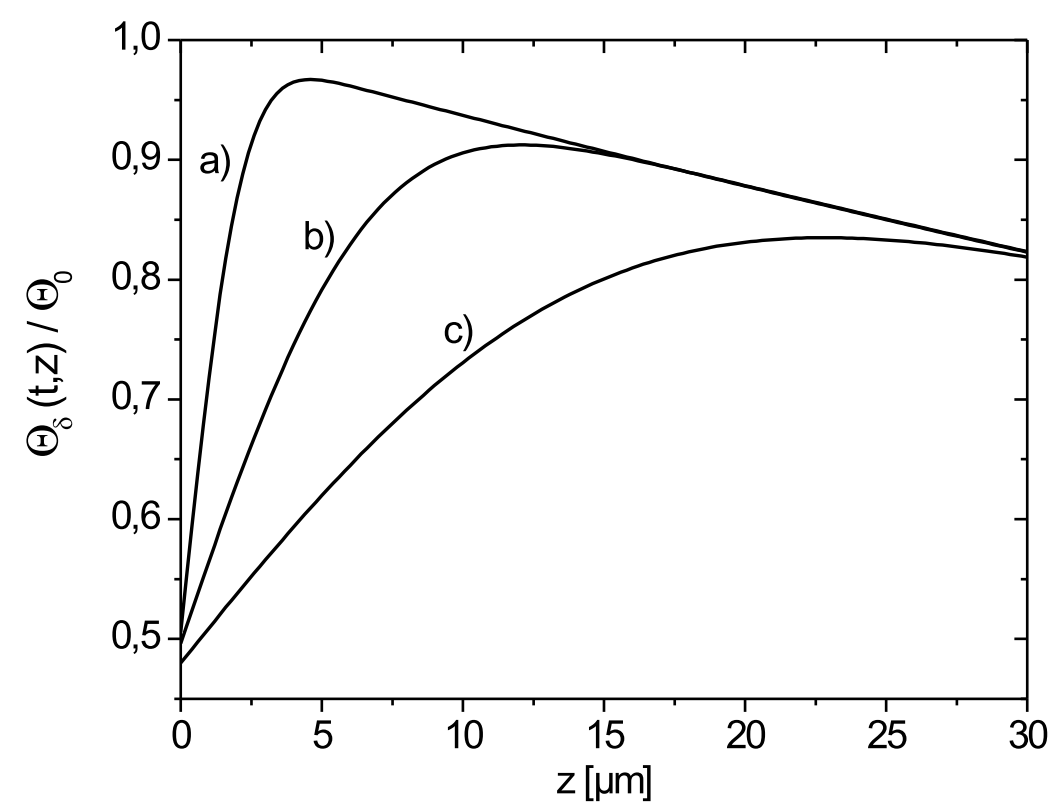

Abb. A.2: Verlauf der (normierten) Impulsantwort $\hat{\Theta}_{\delta}(t, z)=\Theta_{\delta}(t, z) / \Theta_{0}$ im Wasser nach a) $10 \mu \mathrm{s}$, b) $100 \mu \mathrm{s}$ und c) $500 \mu \mathrm{s}$. Das angrenzende Medium ist Quarz. Die Berechnungen erfolgten für eine Absorption $\mu=6.5 / \mathrm{mm}$.

Erwartungsgemäß spiegelt die Impulsantwort bei $t=0$ das initiale Temperaturprofil (A.16) wider. Ein wesentlicher Unterschied besteht aber in der Unstetigkeit bei $z=0$, 
die sich durch den Faktor $(1+K) / 2$ ausdrückt. Hierdurch wird die maximale Temperaturerhöhung $\Theta_{0}$ nicht an der Grenzfläche erreicht, sondern in einer differentiell kleinen Tiefe $\mathrm{d} z$, für die $\exp (-\mu \mathrm{d} z) \approx 1$ angenommen werden kann. Das Verhältnis von $\Theta_{0}$ zur Temperaturerhöhung an der Grenzfläche beträgt gerade $(1+K) / 2$. Im weiteren Verlauf (Abbildung A.2) nimmt der Abstand der maximalen Temperaturerhöhung zur Grenzfläche stetig zu, und es bildet sich dazwischen ein kontinuierlicher Temperaturgradient. Dessen relative Höhe beträgt nur anfänglich $(1+K) / 2$, da die Wärmeleitung zunehmend für den Temperaturausgleich sorgt.

Gemäß Gl. (A.18) setzt sich die Größe $K$ aus den Materialkonstanten $\gamma_{i}$ und $k_{i}$ der angrenzenden Medien zusammen. Für Quarz-Wasser berechnet berechnet sich $K$ zu ungefähr 0.02 , so daß $(1+K) / 2 \approx 0.51$ ergibt. Die Temperaturerhöhung an der Grenzfläche ist damit zu allen Zeiten höchstens halb so hoch wie die maximale Temperaturerhöhung. Für Wasser-Luft beträgt $K$ etwa 0.99 und folglich ist $(1+K) / 2 \approx 1$, was die hohe thermische Isolierfähigkeit von Luft widerspiegelt. Eine vergleichende Darstellung der Impulsantworten zu den beiden $K$-Werten ist in Abbildung A.3 gegeben.

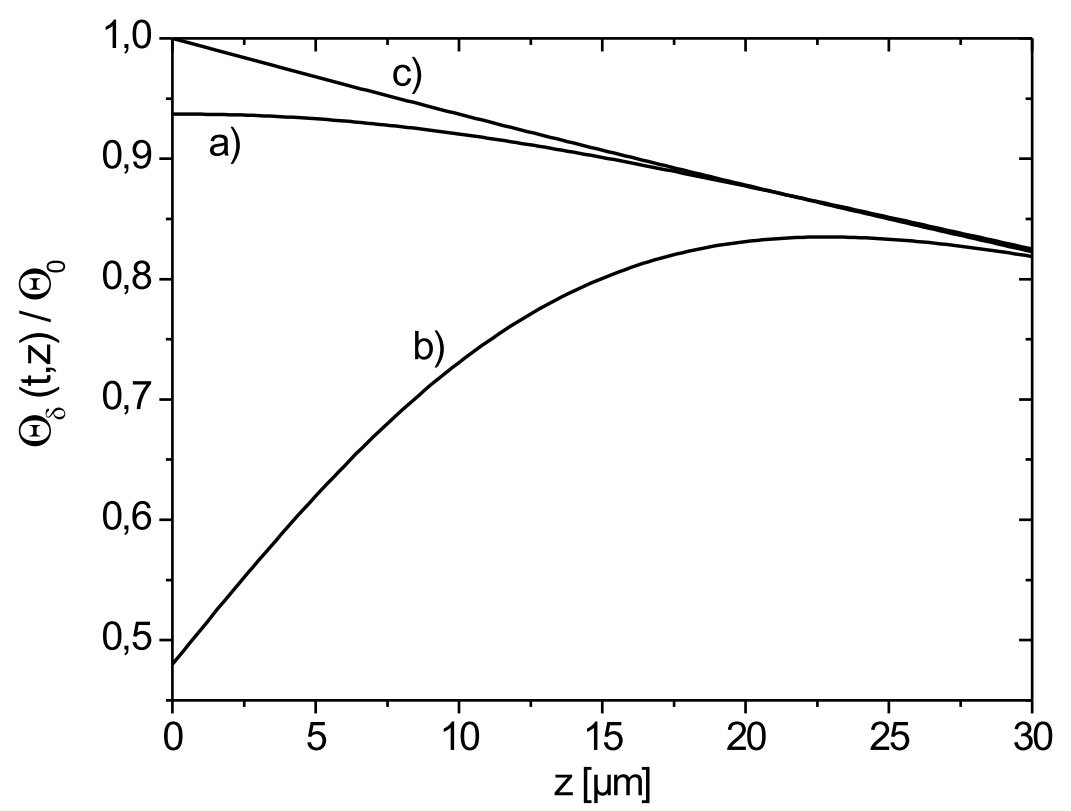

Abb. A.3: Verlauf der (normierten) Impulsantwort $\hat{\Theta}_{\delta}(t, z)=\Theta_{\delta}(t, z) / \Theta_{0}$ im Wasser nach $t=$ $500 \mu \mathrm{s}$ mit a) Luft und b) synthetischem Quarz als angrenzendem Medium. Zum Vergleich zeigt die Kurve c) das initiale Temperaturprofil $\propto \exp (-\mu z)$, mit $\mu=6.5 / \mathrm{mm}$. 


\section{Literaturverzeichnis}

[1] D. Albagli, L. T. Perelman, G. S. Janes, C. v. Rosenberg, I. Itzkan und M. S. Feld, Inertially Confined Ablation of Biological Tissue, Lasers in the Life Sciences 6 (1), 55 (1994).

[2] J. M. Bennett und L. Mattsson, Introduction to surface roughness and scattering, Techn. Ber. UUIP-1185, Uppsala University, Institute of Physics (06/88 1988).

[3] R. T. Beyer, Parameter of Nonlinearity in Fluids, Journal of the Acoustical Society of America 32 (6), 719 (1960).

[4] R. T. Beyer, Nonlinear Acoustics, (U.S. Gov. Print. Off., 1974).

[5] J.-L. Boulnois, Photophysical Processes in Recent Medical Laser Developments: a Review, Lasers in Medical Science 1, 47 (1986).

[6] T. Brendel, Experimentelle Untersuchungen zur thermoelastischen Absorption bei 532 nm, Diplomarbeit, Universität Göttingen (1998).

[7] T. Brendel und R. Brinkmann, Highly resolved tracing of Q-switched mid-IR laser induced vaporization, in Laser-Tissue Interaction XII, herausgegeben von S. Jacques, Bd. 4257, (SPIE, San José, CA, USA, 2001).

[8] T. Brendel und R. Brinkmann, Mid-IR laser induced superheating of water and its quantification by an optical temperature probe, Applied Optics 43 (9), 1856 (2003).

[9] T. Brendel und R. Brinkmann, Forced superheating of water by nanosecond mid-IR laser pulses, eingereicht bei Applied Physics Letters (2004).

[10] R. Brinkmann und C. Hansen, Beam-profile modulation of thulium laser radiation applied with multimode fibers and its effect on the threshold fluence to vaporize water, Applied Optics 39 (19) (2000). 
[11] R. Brinkmann, A. Knipper, G. Dröge, F. Schröer, B. Gromoll und R. Birngruber, Fundamental studies of fiber-guided soft tissue cutting by means of pulsed midinfrared lasers and their application in laser ureterotomy, Journal of Biomedical Optics 3, 85 (1998).

[12] R. Brinkmann, D. Theisen, T. Brendel und R. Birngruber, Single-Pulse 30-J Holmium Laser for Myocardial Revascularization - A Study on Ablation Dynamics in Comparison to CO2 Laser-TMR, IEEE Journal of Selected Topics in Quantum Electronics 5 (4), 969 (1999).

[13] V. P. Carey, Liquid-vapor phase change phenomena: an introduction to the thermophysics of vaporization and condensation processes in heat transfer equipment, Series in chemical and mechanical engineering, (Hemisphere publishing corporation, 1992).

[14] H. S. Carslaw und J. C. Jaeger, Conduction of Heat in Solids, (Clarendon, Oxford, UK, 1959).

[15] A. B. Coppens, R. T. Beyer, M. B. Seiden, J. Donohue, F. Guepin, R. H. Hodson und C. Townsend, Parameter of Nonlinearity in Fluids. II, Journal of the Acoustical Society of America 38, 797 (1965).

[16] P. G. Debenedetti, Metastable liquids: concepts and principles, Physical chemistry: science and engineering, (Princeton university press, 1996).

[17] R. S. Dingus und R. J. Scammon, Grüneisen-stress induced ablation of biological tissue, in Laser-Tissue Interaction II, Bd. 1427, (SPIE, 1991).

[18] Y. Domankevitz, M. S. Lee und N. S. Nishioka, Effects of irradiance and spot size on pulsed holmium laser ablation of tissue, Applied Optics 32 (4), 569 (1993).

[19] F. A. Duck, Physical Properties of Tissue - A Comprehensive Reference Book, (Academic Press, 1990).

[20] S. Egerev und e. al., Sound radiation by a spatially inhomogeneous pulsed volume source, Soviet Physics: Acoustics 32 (2), 103 (1986).

[21] H. Endo, Empirical expression for nonlinarity parameter B/A of liquids, Journal of the Acoustical Society of America 72 (1), 235 (1982). 
[22] M. Fiebig, Diodengepumpte Festkörperlaser im Spektralbereich um $2 \mu$ m für Anwendungen in der Lasermedizin, Doktorarbeit, Universität Hannover (1998).

[23] N. Fletcher, Van der Waals' equation and nucleation theory, European Journal of Physics 14, 29 (1993).

[24] M. Frenz, F. Könz, H. Pratisto und H. P. Weber, Starting mechanisms and dynamics of bubble formation induced by a Ho:Yttrium aluminium garnet laser in water, Journal of Applied Physics 84 (11), 5905 (1998).

[25] M. Frenz, G. Paltauf und H. Schmidt-Kloiber, Laser-Generated Cavitation in Absorbing Liquid Induced by Acoustic Diffraction, Physical Review Letters 76 (19), 3546 (1996).

[26] C. Gerthsen, H. O. Kneser und H. Vogel, Physik, (Springer, 1992), 16. Aufl.

[27] Y. E. Gorbaty und G. V. Bondarenko, Experimental Technique for Quantitative IR Studies of Highly Absorbing Substances at High Temperatures and Pressures, Applied Spectroscopy 53 (8), 908 (1999).

[28] W. Greiner, L. Neise und H. Stöcker, Thermodynamics and statistical mechanics, Classical theoretical physics, (Springer, 1994).

[29] S. Großmann, Mathematischer Einführungskurs für die Physik, Teubner Studienbücher Physik, (B. B. Teubner, 1991).

[30] V. Gusev und A. A. Karabutov, Laser Optoacoustics, (AIP Press, New York, 1993).

[31] G. M. Hale und M. R. Querry, Influence of Temperature on the Spectrum of Water, Journal of the Optical Society of America 62 (9), 1103 (1972).

[32] C. Hansen, R. Brinkmann und R. Birngruber, Influence of Pulse Width and Speckle Formation on the Ablation Thresholds in Water by Means of Pulsed mid-IR Laser Radiation, in Laser-Tissue Interaction and Tissue Optics IV, Bd. 3195, (SPIE/BiOS, San Remo, 1997).

[33] R. Hibst, Technik, Wirkungsweise und medizinische Anwendungen von Holmiumund Erbium-Lasern, Bd. 15 von Fortschritte in der Lasermedizin, (ecomed, Landsberg, 1996). 
[34] The International Association for the Properties of Water and Steam (IAPWS), www.iapws.org, Release on the IAPWS Industrial Formulation 1997 for the Thermodynamic Properties of Water and Steam (1997).

[35] W. Isay, Kavitation, (Schiffahrts-Verlag Hansa C. Schroedter \& Co, 1981).

[36] S. L. Jacques, Laser-Tissue Interactions, Lasers in General Surgery 72 (3), 531 (1992).

[37] E. D. Jansen, Temperature Dependence of the Absorption Coefficient of Water for Midinfrared Laser Radiation, Lasers in Surgery and Medicine 14, 258 (1994).

[38] A. A. Karabutov, N. B. Podymova und V. S. Letokhov, Time-resoveld laser optoacoustic tomography of inhomogeneous media, Applied Physics B 63, 545 (1996).

[39] A. A. Karabutov, O. Rudenko und E. B. Cherepetskaya, Theory of the thermooptical generation of nonsteady acoustic fields, Soviet Physics: Acoustics 25 (3), 218 (1979).

[40] S. G. Kasoev und L. M. Lyamshev, Theory of laser-pulse generation of sound in a liquid, Soviet Physics: Acoustics 23 (6), 510 (1977).

[41] S. G. Kasoev und L. M. Lyamshev, Sound generation in a liquid by laser pulses of arbitrary shape, Soviet Physics: Acoustics 24 (4), 302 (1978).

[42] A. Kiesel, A. Duparré, M. Haase, F. Corinad und H. Truckenbrodt, Surface Finish Assessment of Synthetic Quartz Glass, in Specification, Production, and Testing of Optical Components and Systems, Bd. 2775, (SPIE, 1996).

[43] R. T. Knapp, Cavitation, (McGraw-Hill, 1970).

[44] H. Kuttruff, Physik und Technik des Ultraschalls, (Hirzel Verlag, Stuttgart, 1988).

[45] H. M. Lai und K. Young, Theory of the pulsed optoacoustic technique, Journal of the Acoustical Society of America 72 (6), 2000 (1982).

[46] L. D. Landau und E. M. Lifschitz, Hydrodynamik, Bd. 6 von Lehrbuch der theoretischen Physik, (Harri Deutsch, 1991).

[47] B. I. Lange, T. Brendel und G. Hüttmann, Temperature dependence of the absorption in water of light at holmium and thulium laser wavelengths, Applied Optics (2002). 
[48] W. Lauterborn, T. Kurz und M. Wiesenfeldt, Coherent Optics, Fundamentals and Applications, (Springer, 1995).

[49] T. G. Leighton, The acoustic bubble, (Academic Press, 1994).

[50] W. A. P. Luck, Zur Assoziation des Wassers III, Berichte der Bunsengesellschaft 69 (7), 626 (1965).

[51] L. M. Lyamshev, Optoacoustic sources of sound, Soviet Physics Uspekhi 24 (12) (1981).

[52] L. M. Lyamshev und K. A. Naugol'nykh, Optical generation of sound: nonlinear effects (review), Soviet Physics: Acoustics 27 (5), 357 (1981).

[53] L. M. Lyamshev und L. V. Sedov, Optical generation of sound in a liquid: thermal mechanism (review), Soviet Physics: Acoustics 27 (1), 4 (1981).

[54] A. Miotello und R. Kelly, Critical assessment of thermal models for laser sputtering at high fluences, Applied Physics Letters 67 (24), 3535 (1995).

[55] C. E. Mortimer, Chemie, (Georg Thieme Verlag, Stuttgart, 1987).

[56] K. A. Mørch und J. P. Song, Cavitation nuclei at solid-liquid interfaces, in Proc. Cavitation, Bd. C453/059, S. 1-7, (Institution of Mechanical Engineers, London, UK, 1992).

[57] M. Mrochen, K. Vogler und N. Glaeske, Experiments on Q-switched Solid State Lasers in the $2 \mu \mathrm{m}-3 \mu \mathrm{m}$ Spectral Region, Laser und Optoelektronik 28 (1), 42 (1996).

[58] K. Nahen, Akustische Online-Kontrolle der Infrarot-Photoablation biologischer Gewebe, Doktorarbeit, Medizinische Universität zu Lübeck (2001).

[59] W. Nolting, Elektrodynamik, Grundkurs: Theoretische Physik, (ZimmermannNeufang, Ulmen, 1993).

[60] Y. Ohde, M. Ikemizu, H. Okamoto, T. Yokoyama und S. Shibata, Cavitation history effect of a water-metal Berthelot tube system interpreted by an elaborated gastrapping crevice model, Journal of Physics D: Applied Physics 22, 1721 (1989). 
[61] Y. Ohde, H. Kazuki, M. Ono, H. Isono und H. Watanabe, Effect on trends in negative pressure of surface pre-treatments for the sealing plugs of a water-metal Berthelot tube system, Journal of Physics D: Applied Physics (1992).

[62] A. A. Oraevsky und S. L. Jacques, Mechanism of laser ablation for aqueous media irradiated under confined stress conditions, Journal of Applied Physics 78 (2), 1281 (1995).

[63] G. Paltauf, E. Reichel und H. Schmidt-Kloiber, Study of different ablation models by use of high-speed-sampling photography, in Laser-Tissue Interaction III, Bd. 1646, S. 343-352, (SPIE, 1992).

[64] G. Paltauf und H. Schmidt-Kloiber, Model study to investigate the contribution of spallation to pulsed laser ablation of tissue, Lasers in Surgery and Medicine 16, 277 (1995).

[65] G. Paltauf und H. Schmidt-Kloiber, Microcavity dynamics during laser-induced spallation of liquids and gels, Applied Physics A 62, 303 (1996).

[66] G. Paltauf und H. Schmidt-Kloiber, Measurement of laser-induced acoustic waves with a calibrated optical transducer, Journal of Applied Physics 82 (4), 1525 (1997).

[67] G. Paltauf und H. Schmidt-Kloiber, Photoacoustic waves excited in liquids by fibertransmitted laser pulses, Journal of the Acoustical Society of America 104 (2), 890 (1998).

[68] G. Paltauf und H. Schmidt-Kloiber, Laser-induced acoustic damage in and around sub-millimeter-sized optical absorbers, in CLEO/Europe - EQEC Focus Meetings, S. 130-132, (OSA, Munich, Germany, 1999).

[69] L. W. Pinkley, P. Sethna und D. Williams, Optical constants of water in the infrared: Influence of temperature, Journal of the Acoustical Society of America 67 (4), 494 (1977).

[70] M. Platte, Ultraschallwandler aus dem piezoelektrischen Hochpolymer Polyvinylidenflourid, Doktorarbeit, RWTH Aachen (1984).

[71] S. Ridah, Shock waves in water, Journal of Applied Physics 64 (1) (1988). 
[72] V. M. Ristic, Radiation and Diffraction of Acoustic Waves, Planar Radiators, and Acoustic Lenses, in Principles of Acoustic Devices, S. 294-341, (John Wiley and Sons, 1983).

[73] P. Schiebener, J. Straub, J. M. H. Levelt Sengers und J. S. Gallagher, Refractive Index of Water and Steam as Function of Wavelength, Temperature and Density, Journal of Physical and Chemical Reference Data 19 (3), 677 (1990).

[74] P. Schmidt, Minimierung thermomechanischer Nebenwirkungen bei arthroskopischer Chirurgie durch Verwendung gütegeschalteter IR-Mehrfachpulse, Forschungsbericht, Medizinisches Laserzentrum Lübeck (2000).

[75] Eigenschaften des synthetischen Quarzes SQ1, Datenblatt, Sico Jena (1999).

[76] M. W. Sigrist und F. K. Kneubühl, Laser-generated stress waves in liquids, Journal of the Acoustical Society of America 64 (6), 1652 (1978).

[77] J. Steinert, S. Gliech, A. Wuttig, A. Duparré und H. Truckenbrodt, Advanced Methods for Surface and Subsurface Defect Characterization of Optical Components, in Optical Metrology Roadmap for the Semiconductor, Optical, and Data Storage Industries, herausgegeben von G. A. Al-Jumaily, A. Duparré und B. Singh, Bd. 4099, S. 290-298, (SPIE, 2000).

[78] A. A. Tesar, B. A. Fuchs und P. P. Hed, Examination of the polished surface character of fused silica, Applied Optics 31 (34), 7164 (1992).

[79] V. Venugopalan, N. S. Nishioka und B. B. Mikic, Thermodynamic Response of Soft Biological Tissues to Pulsed Infrared Laser Irradiation, Biophysical Journal 70, 2981 (1996).

[80] V. L. Vodopyanov, Bleaching of water by intense light at the maximum of $\lambda \sim 3 \mu \mathrm{m}$ absorption band, Soviet Physics JETP 70, 114 (1990).

[81] A. Vogel und V. Venugopalan, Mechanisms of Pulsed Laser Ablation of Biological Tissues, Chemical Reviews 103, 577 (2003).

[82] B. Wolfrum, R. Mettin, T. Kurz und W. Lauterborn, Observations of pressurewave-excited contrast agent bubbles in the vicinity of cells, Applied Physics Letters 81 (26), 5060 (2002). 
[83] A. Wuttig, J. Steinert, A. Duparré und H. Truckenbrodt, Surface roughness and subsurface damage characterization of fused silica substrates, in EUROPTO, Bd. 3739, S. 369-376, (SPIE, Berlin, 1999).

[84] A. D. Yablon, N. S. Nishioka und B. B. Mikic, Measurement of tissue absorption coefficients by use of interferometric photothermal spectroscopy, Applied Optics 38 (7), 1259 (1999).

[85] F. R. Young, Cavitation, (McGraw-Hill, 1989).

[86] G. N. Zatsepina, Physical Properties and Structure of Water, (Moscow State University, 1987). 


\section{Wissenschaftlicher Lebenslauf}

\section{Tobias Brendel}

geboren am 09.07.1971 in Verden

Staatsangehörigkeit: deutsch

1984 - 1991 Domgymnasium Verden

05/1991 Abitur, Gesamtnote: 1.6

1992 - 1998 Studium der Physik (Diplom) an der Georg-August-Universität zu Göttingen

07/94 Vordiplom in Physik, Gesamtnote: 1.6

04/95 - 09/95 Auslandssemester an der Université Claude-Bernad de Lyon, Frankreich

05/98 Diplom in Physik an der Georg-August-Universität zu Göttingen Gesamtnote: 1.2

07/98 - 07/02 Wissenschaftliche Assistenz an der Medizinisches Laserzentrum Lübeck GmbH

02/03 - 06/04 Promotion am Dritten Physikalischen Institut der Universität zu Göttingen Arbeitsgruppe Prof. Dr. Werner Lauterborn 